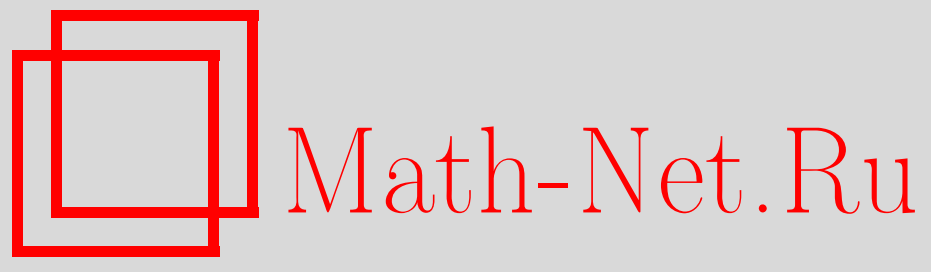

А. Ю. Васильев, А. Г. Сергеев, Классические и квантовые пространства Тейхмюллера, УМН, 2013, том 68, выпуск 3, 39-110

DOI: https://doi.org/10.4213/rm9517

Использование Общероссийского математического портала Math-Net.Ru подразумевает, что вы прочитали и согласны с пользовательским соглашением http://www . mathnet.ru/rus/agreement

Параметры загрузки:

IP: 54.198 .64 .247

26 апреля 2023 г., 17:07:27

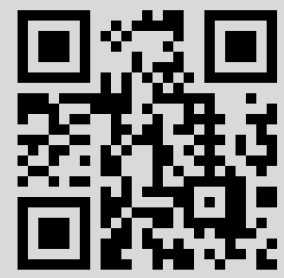




\section{Классические и квантовые пространства Тейхмюллера}

\section{А. Ю. Васильев, А. Г. Сергеев}

Теория Тейхмюллера - активно развивающаяся и разветвленная область математики, имеющая многочисленные связи как с другими направлениями математической науки, так и с ее приложениями, в первую очередь в теоретической физике. В данном обзоре представлены в исторической ретроспективе основные направления развития указанной теории и ее приложения к теории струн.

Библиография: 128 названий.

Ключевые слова: пространство Тейхмюллера, риманова поверхность, квазиконформное отображение, модуль семейства кривых, дифференциал Бельтрами, квазисимметричный гомеоморфизм, универсальное пространство Тейхмюллера, геометрическое квантование, некоммутативная геометрия.

DOI: $10.4213 / \mathrm{rm} 9517$

\section{СОДЕРЖАНИЕ}

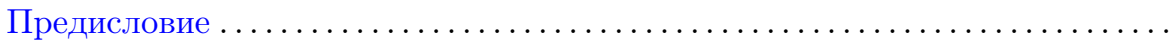

1. Историческое введение: главные действующие лица теории Тейхмюлле-

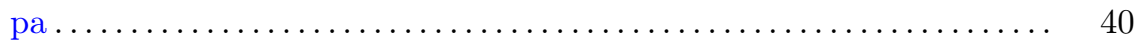

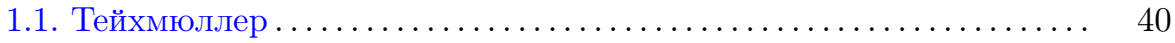

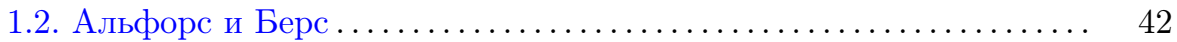

2. Пространство Тейхмюллера компактных римановых поверхностей.... 45

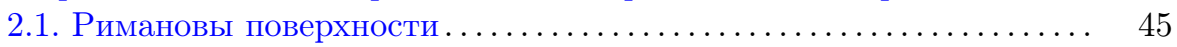

2.2. Квазиконформные отображения .................... 48

2.3. Определение пространства Тейхмюллера, его касательное и ко-

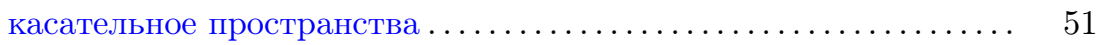

2.4. Теорема Тейхмюллера........................... 54

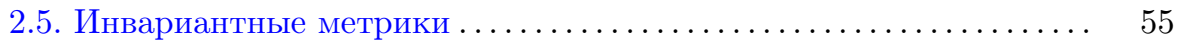

2.6. Пространство модулей Римана........................ 56

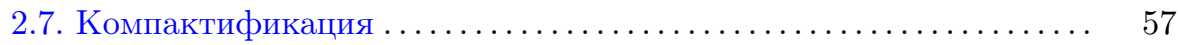

2.8. Модули семейств кривых на пространствах Тейхмюллера...... 59

2.9. Дополнение: пространства Тейхмюллера и теория струн.... 63

При подготовке этой статьи первый автор пользовался поддержкой Норвежского исследовательского совета (гранты № 204726/V30, 213440/BG), второй автор - РФФИ (гранты № 10-01-00178, 11-01-12033-офи-м-2011), программы "Ведущие научные школы" (грант НШ-7675.2010.1) и научной программы Президиума РАН "Нелинейная динамика".

(C) А. Ю. ВАСильев, А. Г. СЕргеЕв, 2013 


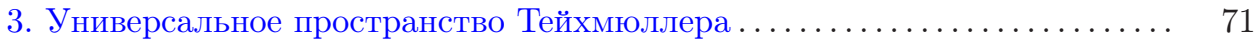

3.1. Квазисимметричные гомеоморфизмы ................. 71

3.2. Определение универсального пространства Тейхмюллера ...... 73

3.3. Свойства универсального пространства Тейхмюллера......... 76

3.4. Подпространства универсального пространства Тейхмюллера .. 81

3.5. Грассманова реализация универсального пространства Тейхмюллера ............................... 84

3.6. Квантование пространства нормализованных диффеоморфизмов 92

3.7. Квантование универсального пространства Тейхмюллера ..... . 100

Список литературы ...................................... 104

\section{Предисловие}

В 1939 г. Освальд Тейхмюллер предложил классификацию римановых поверхностей, основанную на их параметризации точками множества, известного теперь под названием пространства Тейхмюллера. Теория Тейхмюллера является активно развивающейся и разветвленной областью математики, имеющей многочисленные связи с другими направлениями математической науки, в первую очередь, математической физикой. Она включает в себя достижения многих математических и физических дисциплин, таких как комплексный анализ и гиперболическая геометрия, теория дискретных групп и алгебраическая геометрия, топология и дифференциальная геометрия, теория групп Ли и симплектическая геометрия, теория чисел и теория динамических систем, топологическая квантовая теория поля и теория струн, и т. д. В данной статье мы попытались представить основные направления развития указанной теории, начиная с первых работ Тейхмюллера и кончая современными результатами. Учитывая, что этой теме посвящено несколько книг, в журнальном обзоре трудно претендовать на всеобъемлющий охват достижений теории Тейхмюллера. Поэтому нашей целью было представить в исторической ретроспективе те разделы теории, которые представляют интерес, в первую очередь, для нас самих.

\section{1. Историческое введение: главные действующие лица теории Тейхмюллера}

1.1. Тейхмюллер. В своей знаменитой работе [93] Георг Фридрих Бернхард Риман (1826-1866) подсчитал число параметров для классов изоморфизмов алгебраических уравнений от двух комплексных переменных или, другими словами, классов компактных римановых поверхностей с точностью до биголоморфной эквивалентности. Это привело его к определению того, что называется теперь грубым пространством модулей, т. е. к глобальному пространству параметров комплексных структур на двумерных поверхностях. Примерно восемьдесят лет спустя Тейхмюллер осознал, что задачи классификации становятся более доступными, если снабдить рассматриваемые объекты дополнительной структурой, так называемым оснащением. Его идея состояла в том, чтобы сначала классифицировать “оснащенные объекты”, а затем описать отношения эквивалентности, возникающие при “забывании” оснащения. 
Остановимся коротко на биографии Тейхмюллера и других “действующих лиц" рассматриваемой в этом обзоре теории.

Пауль Юлиус Освальд Тейхмюллер (1913-1943) поступил в Гёттингенский университет летом 1931 г. с целью изучения математики и немедленно проявил себя как незаурядный студент. Его наставником стал Хельмут Хассе (1898-1979), возглавивший после Германа Вейля его кафедру в Гёттингене в 1934 г. Тейхмюллер выбрал Хассе в качестве своего научного руководителя несмотря на то, что его математические пристрастия лежали вдали от направления, развивавшегося Хассе.

K несчастью, интересы Тейхмюллера не ограничились одной математикой. Находясь в Гёттингене, он подпал под влияние нацистской пропаганды и присоединился к национал-социалистической партии (NSDAP). Вступив в число штурмовиков SA (Sturm Abteilung), он стал одним из лидеров нацистски настроенных студентов. Второго ноября 1933 г. именно Тейхмюллер возглавил студенческий бойкот лекций Эдмунда Ландау в духе пресловутого гитлеровского "Закона о реорганизации гражданской службы”.

В 1935 г. Тейхмюллер защитил диссертацию на соискание степени Doktor der Naturwissenschaften и в 1936 г., переехав в Берлин, начал работать над докторской диссертацией (Habilitationsschrift) под патронажем Людвига Бибербаха (1886-1982). Эта диссертация [106] была защищена им в марте 1938 г. (Заметим в скобках, что Бибербах относился с неодобрением к ранним алгебраическим работам Тейхмюллера, написанным под влиянием Хассе.)

В период с 1935 по 1943 г., т. е. менее чем за 10 лет, он опубликовал 34 статьи, во многом предвосхитившие впечатляющее развитие теории римановых поверхностей в течение последующих 70 лет. Интересы Тейхмюллера охватывали широкий круг математических дисциплин от математической логики, через алгебру, теорию чисел и теорию функций, до топологии и дифференциальной геометрии. Начиная с докторской диссертации [106] и последующей статьи [107], а затем в известной работе [108] и ее дополнении [109] им были заложены основы того, что называется теперь теорией пространств Тейхмюллера. Одна из последних статей [110], развивающих указанную теорию, была опубликована уже после смерти Тейхмюллера.

Среди 34 работ, принадлежащих Тейхмюллеру, 21 работа опубликована в "Deutsche Mathematik" - математическом журнале, основанном Людвигом Бибербахом и Теодором Валеном в 1936 г. "с целью поддержки германской математики в противовес еврейской". Из-за того, что большинство работ Тейхмюллера было опубликовано в этом журнале, прекратившем свое существование после Второй мировой войны, они оставались практически недоступными до 1986 г., когда Ларс Альфорс и Фредерик Геринг выпустили том [111] с избранными статьями Тейхмюллера.

Будучи членом национал-социалистической партии, Тейхмюллер принял активное участие в сражениях Второй мировой войны, начиная с гитлеровской оккупации Норвегии в 1940 г. В начале 40-х годов он занялся под влиянием Бибербаха криптографией и даже читал лекции на эту тему в Берлине в 1942 г. Но после поражения нацистов под Сталинградом Тейхмюллер снова вернулся в армию и был послан на Восточный фронт, где был убит 11 сентября 1943 г. около Полтавы после сражения под Харьковом. 
Более подробные сведения о работе Тейхмюллера и его жизни можно почерпнуть из публикаций [4], [81], [95], а также статьи [23].

Переходя к публикациям по теории Тейхмюллера, начнем с уже упомянутого нами собрания статей самого Тейхмюллера [111]. Помимо этого, укажем "Настольную книгу по теории Тейхмюллера" [84], в двух первых томах которой дается (более чем 1500-страничный) глубокий и обстоятельный обзор теории Тейхмюллера. Несколько монографий, частично или полностью, посвящены различным аспектам теории Тейхмюллера, см., например, [3], [9], [30], [32], [33], [43], [45], [58], [62], [66], [71], [97], [113], [118]. Как это ни удивительно, имеется не так много обзорных статей, освещающих современные достижения этой теории. Отметим в этой связи обзор [85], относящийся в основном к универсальному пространству Тейхмюллера, введенному Берсом [17] в 1968 г.

1.2. Альфорс и Берс. Как известно, еще Риман [93] заметил, что классы конформной эквивалентности поверхностей рода $g$ зависят от $6 g-6$ вещественных параметров. Тейхмюллер [108] в 1939 г. продолжил исследование (в основном на эвристическом уровне) указанных классов эквивалентности на основе совершенно иной идеи. А именно, предположим, что $S_{1}$ и $S_{2}$ - две римановых поверхности рода $g$, гомеоморфных друг другу, и $f$ - гомеоморфизм $S_{1}$ на $S_{2}$. Допустим, что поверхности $S_{1}$ и $S_{2}$ конформно не эквивалентны друг другу. В этом случае обязательно существуют квазиконформные отображения $S_{1}$ на $S_{2}$, гомотопные $f$. Среди таких отображений существует единственное отображение $f_{0}$, минимизирующее вещественную дилатацию $K(f)$ (характеризующую максимальное отклонение отображения $f$ от конформного отображения). Отождествим поверхности $S_{1}$ и $S_{2}$ с точками метрического пространства, наделенного метрикой $(1 / 2) \log K(f)$. Указанное метрическое пространство локально гомеоморфно $(6 g-6)$-мерному евклидову пространству. Тейхмюллеру удалось доказать в работе [108] единственность, а затем, в 1943 г. [109], и существование указанного отображения $f_{0}$.

Следует отметить, что оригинальные доказательства Тейхмюллера были по большей части эвристическими и содержали серьезные пробелы. Альфорс и Берс потратили немало усилий на то, чтобы довести эти доказательства до строгих рассуждений. Вначале Альфорс в работе [7] 1953 г. предложил собственное доказательство теоремы существования, затем Берс в [16] сумел перевести эвристические исходные идеи Тейхмюллера в плоскость строгой математики. Московское издательство "Иностранная литература" выпустило в 1961 г. сборник [11], в который вошли доклад Берса на Эдинбургском математическом конгрессе (1958) и несколько докладов с конференции по аналитическим функциям, состоявшейся в Институте перспективных исследований в Принстоне в сентябре 1957 г. (перевод В.А. Зорича и А. А. Кириллова, редакция Б. В. Шабата). Кроме того, в него были включены принадлежащее Альфорсу доказательство теоремы существования [7] и несколько коротких статей Берса. Указанный сборник отражал, тем самым, состояние теории Тейхмюллера на тот момент времени.

Воздействие работ Альфорса и Берса на математическое сообщество было столь велико, что в образовательном центре Нью-Йоркского университета (CUNY) в 1955 г. была учреждена серия коллоквиумов (собиравшихся раз в три 
года), посвященных математическому наследию Альфорса и Берса, под общим названием коллоквиумов Берса. После кончины Ларса Альфорса их название было изменено на коллоквиумы Альфорса и Берса. (Первый коллоквиум с этим новым названием состоялся в Нью-Йоркском университете в Стони Брук в 1998 г.)

Ларс Валериан Альфорс родился в Гельсингфорсе (Хельсинки), Финляндия, в 1907 г. в шведской семье. Его отец был профессором инженерии в Политехническом институте, мать трагически скончалась во время родов. В 1924-1928 гг. Альфорс изучает математику в Гельсингфорсском университете, где его учителями были Линделёф и Неванлинна. В 1928-1929 гг. Неванлинна был приглашен заведующим кафедры Германа Вейля в Федеральной высшей технической школе (Eidgenössische Technische Hochschule) в Цюрихе и пригласил Альфорса присоединиться к нему. Затем, вместе с Неванлинной, Альфорс переезжает в Париж в 1930 г., где остается до тех пор, пока ему не предлагают место лектора по математике в Турку. В том же 1930 г. Альфорс представляет свою докторскую диссертацию и отправляется путешествовать по Европе. В 1935 г. он встречается с Каратеодори, который направляет его в Гарвардский университет на трехлетнюю стажировку. Однако, испытывая тоску по родине, Альфорс в 1938 г. возвращается в Финляндию, как только ему предлагают место заведующего кафедрой математики в Хельсинкском университете. В начале второй мировой войны семья Альфорса переезжает в Швецию.

Находясь в Швеции, Альфорс встречает Арне Бёрлинга, который неоднократно оказывал ему большую помощь. С Арне Бёрлингом его связывала впоследствии многолетняя дружба, которая продолжалась до самой смерти Бёрлинга в 1986 г. В этой дружбе, однако, случился длительный перерыв, произошедший при следующих обстоятельствах. Будучи весьма щепетильным ученым, Бёрлинг никогда не публиковал свои результаты, не убедившись в их абсолютной правильности. Поэтому, когда Альфорс в одной из своих лекций упомянул о недавней работе, выполненной совместно с Бёрлингом, последний был возмущен этим фактом, считая работу незавершенной. Несмотря на то что Альфорс упомянул об их результате с целью подчеркнуть роль Бёрлинга в его доказательстве, отношения между учеными охладились и восстановились только за два года до смерти Бёрлинга.

В 1944 г. Альфорсу предлагают место заведующего кафедрой в Цюрихе. Однако осуществить путешествие в Цюрих в 1944 г. Альфорсу не удается, и только в 1945 г. он добирается до него через Прествик, Глазго, Лондон и Париж. Пребывание в Цюрихе не приносит Альфорсу удовлетворения, поскольку, как он писал, послевоенная пора была не лучиим временем для иностраниа, желающего осесть в Швейиарии. Поэтому он с радостью принимает предложение из Гарварда, где работает вплоть до своей отставки в 1977 г. Скончался Альфорс 11 октября 1996 г. от пневмонии в Питтсфилде, Массачусетс, в возрасте 89 лет. Его книги "Комплексный анализ" (1953), "Римановы поверхности" (вместе с Л. Сарио) (1960), "Лекции по квазиконформным отображениям” (1966) и "Конформные инварианты" (1973) не утратили своего значения до сих пор, так же как и статьи, которых насчитывается более сотни.

Альфорс был отмечен несколькими наградами за свой выдающийся вклад в математику. Среди них первая Филдсовская медаль (1936) за работы по 
теории римановых поверхностей и квазиконформным отображениям, врученная ему всего лишь через четыре года после кончины Джона Филдса. Другой почетной наградой явилась премия Вольфа, присужденная ему в 1981 г. Альфорсу принадлежат основополагающие результаты по теории мероморфных кривых, теории распределения значений, теории римановых поверхностей, конформной геометрии, теории квазиконформных отображений, теории клейновых групп и другим направлениям математики.

Липман Берс (друзья называли его Липой) родился в еврейской учительской семье в Риге 23 мая 1914 г. Во время Первой мировой войны и революции в России Латвия оказалась вовлеченной в гражданскую войну и семья Берсов переехала в Санкт-Петербург (называвшийся в то время Петроградом), однако в 1918 г. вернулась в Латвию, когда последняя обрела независимость. После короткого пребывания в Цюрихе Берс возвратился в Рижский университет. 15 мая 1934 г. Ульманис совершил бескровный переворот, установив в Латвии националистическую диктатуру, просуществовавшую до 1940 г. Поскольку Берс придерживался социал-демократических взглядов и активно боролся за гражданские права, было издано постановление о его аресте, из-за чего ему пришлось бежать в Прагу. Подруга Берса Мария Каган последовала за ним в Прагу, где вступила с ним в брак 15 мая 1938 г.

В том же 1938 г. Берс получил докторскую степень в Карловом университете в Праге под руководством Карела Лёвнера (вошедшего в историю математики, после эмиграции в США, под именем Чарльза Лёвнера). Однако сотрудничество с Лёвнером не удовлетворяло Берса, поскольку он не ощущал поддержки с его стороны. Берс вспоминал, что только впоследствии он оценил особенности преподавательской методики Лёвнера. Он предоставлял каждому из его студентов ровно столько помощи, сколько тому было необходимо.. Очевидно, Лёвнер считал, что Берс не сильно нуждался в его поддержке (см. [82]). Поскольку оба, Лёвнер и Берс, были еврейского происхождения, оставаться в Праге после оккупации Чехословакии нацистами в 1939 г. было опасно для обоих. Лёвнер в результате попал в тюрьму, а Берс находился в розыске в Латвии. Поэтому он предпочел переехать в Париж, где родилась его дочь Руфь. Нацистские армии продвигались все ближе к Парижу, поэтому Берс обратился за визой в американское посольство, но получить ее не успел, так как был вынужден бежать в неоккупированную часть Франции.

Берс и его семья прибыли в Соединенные Штаты только в 1940 г., где воссоединились с его матерью, уже находившейся в Нью-Йорке. Нельзя сказать, что ученых из нацистской Германии и с других оккупированных территорий принимали в США с распростертыми объятиями. Свободных позиций в университетах не хватало, и даже наиболее талантливые из приезжих ученых не могли найти работу по специальности. По этой причине Липа оставался безработным до 1942 г., когда ему предложили должность инструктора-исследователя (Research Instructor) в Университете Брауна. В 1945-1949 гг. Берса и Лёвнера приглашают в Сиракузский университет. В это время руководителем Отделения математики этого университета был назначен Уильям Мартин из Массачусетского технологического института. В его задачу входила реорганизация указанного Отделения, до того рассеянного по школам искусства и науки, бизнеса и инженерии. Этот период оказался для Отделения временем процветания 
благодаря тому, что Мартин пригласил туда целую группу прекрасных математиков, в их числе Лёвнера, Берса и Гелбарта из Университета Брауна, а также Генри Шеффе, Ганса Самельсона, Пауля Халмоша, Рут Гудман, Катрин Морган, Пола Джилберта и Пола Розенблюма.

В эти годы основной темой научной деятельности Берса стала проблема устранения особенностей нелинейных эллиптических уравнений. Полученные результаты были представлены им на Международном конгрессе математиков в 1960 г., а его работа [15] опубликована в "Annals of Mathematics" в 1951 г. В течение 1949-1951 гг. Берс находится в Институте перспективных исследований в Принстоне, где начинается его десятилетняя математическая одиссея, которая в итоге приводит его к теории псевдоаналитических функций и эллиптических уравнений, квазиконформным отображениям, теории Тейхмюллера и клейновым группам. В 1951 г. он переходит в Курантовский институт при Нью-Йоркском университете, а в 1964 г. - в Колумбийский университет, где и остается вплоть до своей отставки в 1984 г. Скончался Липман Берс 29 октября 1993 г. после длительной борьбы с болезнью Паркинсона.

Им получены основополагающие результаты сразу в нескольких областях математики, включая уравнения в частных производных, квазиконформные отображения, теорию Тейхмюллера и клейновы группы (более подробно об этом см. в [5]). В течение жизни он занимал целый ряд престижных математических постов, в частности, был вице-президентом Американского математического общества с 1963 по 1965 г. и президентом этого общества с 1975 по 1977 г. Награжден премией Стила (1975), в 1967 г. был приглашен Лондонским математическим обществом для выступления с первой именной лекцией Харди. Берс принимал активное участие в общественной и политической жизни, борясь за политические и гражданские права.

\section{2. Пространство Тейхмюллера компактных римановых поверхностей}

2.1. Римановы поверхности. История римановых поверхностей начинается, по-видимому, с 1842 г., когда Карл Вейерштрасс сформулировал приниип аналитического продолжсения [119]. В своей докторской диссертации (Doktor der Naturwissenschaften) [92] Риман, научным руководителем которого был Гаусс, впервые ввел в комплексную теорию функций топологические методы, положив тем самым начало теории поверхностей, известных ныне под его именем. В отличие от вейерштрассовского подхода, в основе определения Римана лежало понятие накрытия. (Любопытную попытку объяснить концепцию Римана широкой аудитории предпринял в 1865 г. Карл Нейман [77].)

Теория римановых поверхностей развивалась далее Феликсом Клейном, который в 1882 г. ввел в математический обиход понятие атласа [55]. В работе [86] 1881 г. и последующих публикациях Анри Пуанкаре заложил основы теории униформизации и группового подхода в теории римановых поверхностей. Адольф Гурвиц навел мосты между теорией римановых поверхностей и теорией алгебраических кривых [44]. Сильнейшее воздействие на дальнейшее развитие теории оказала книга Германа Вейля "Понятие римановой поверхности" ("Die Idee der Riemannschen Fläche") 1913 г. [121]. В ней систематическим 
образом были введены такие понятия, как накрывающая поверхность, группа послойных преобразований, односвязная область, род.

В дальнейшем многие известные математики внесли свой вклад в развитие теории римановых поверхностей. Нельзя не вспомнить о роли таких математиков, как Кёбе, Радо, Бенке, Штейн, Стоилов, Спрингер и т.д. В частности, Тибор Радо [91] в 1925 г. впервые предложил определение римановой поверхности как топологической поверхности с комплексной структурой и доказал триангулируемость таких поверхностей. В течение следующей четверти столетия теория абстрактных римановых поверхностей испытала небывалый подъем. Выдающийся вклад в нее принадлежит Рольфу Херману Неванлинне (1895-1980), одному из наиболее известных финских математиков. В своей книге [78] "Униформизация" ("Uniformisierung") 1953 г., основанной на курсах лекций, прочитанных им в Цюрихе и Хельсинки, Неванлинна представил в концентрированном виде современную ему концепцию абстрактных римановых поверхностей, сосредоточившись в основном на случае некомпактных "открытых" римановых поверхностей.

Риманова поверхность $S$ есть связное топологическое хаусдорфово пространство $M$ с заданными на нем открытым покрытием $\left\{U_{j}\right\}$ и системой гомеоморфизмов $\left\{g_{j}\right\}, g_{j}: U_{j} \rightarrow \mathbb{C}$, таких, что на непустых пересечениях $U_{j} \cap U_{k}$ композиции $g_{j} \circ g_{k}^{-1}$ конформны. Говорят, что множество пар $(\kappa a p m)\left\{U_{j}, g_{j}\right\}$ задает на $S$ конформную структуру. Две структуры $\left\{U_{j}^{1}, g_{j}^{1}\right\}$ и $\left\{U_{j}^{2}, g_{j}^{2}\right\}$ называются эквивалентными, если их объединение также задает конформную структуру на $S$. Таким образом, две римановы поверхности с эквивалентными конформными структурами и общим базовым топологическим пространством $M$ определяют одну и ту же риманову поверхность. Пространство $S$ можно также представлять себе как связную компоненту 1-мерного комплексного аналитического многообразия, которая является, тем самым, ориентированным многообразием. Любая конечная компактная риманова поверхность топологически эквивалентна сфере с конечным числом ручек, при этом число $g$ этих ручек называется родом поверхности. По самому определению, род является топологическим инвариантом поверхности.

Риманова поверхность $S$ с краем представляет собой связное многообразие $M$ с краем, внутренность которого наделена структурой римановой поверхности.

Важнейшую роль в теории римановых поверхностей играет фундаментальная группа поверхности. Фиксируем точку $p \in S$ и рассмотрим класс $[c]$ гомотопных замкнутых кривых на поверхности $S$ (т. е. непрерывных отображений из отрезка $[0,1]$ в $S$ ) с совпадающими началом и концом в точке $p$ и заданной ориентацией. На кривых из класса $[c]$ определены операция композиции $c_{1} c_{2}$ петель $c_{1}$ и $c_{2}$, задаваемая последовательным обходом петель с учетом ориентации, и операция обращения $c^{-1}$, задаваемая обходом кривой $c$ в обратном направлении. Эти операции продолжаются до соответствующих операций на гомотопических классах $\left[c_{1}\right]\left[c_{2}\right]=\left[c_{1} c_{2}\right],[c]^{-1}=\left[c^{-1}\right]$. Множество указанных классов с заданными на нем операциями называется фундаментальной группой $\pi_{1}(S, p)$ поверхности $S$ с точкой отсчета $p$. Кривая $\gamma$, соединяющая точку $p$ с другой точкой $p_{1}$, задает изоморфизм $\left[\gamma c \gamma^{-1}\right]$ фундаментальных групп 
$\pi_{1}(S, p)$ и $\pi_{1}\left(S, p_{1}\right)$. С учетом этого изоморфизма можно говорить о единой фундаментальной группе $\pi_{1}(S)$ поверхности $S$.

По любой подгруппе $F$ фундаментальной группы $\pi_{1}(S)$ заданной поверхности $S$ можно построить новую риманову поверхность $S_{F}$, являющуюся неограниченной накрывающей поверхностью над $S$ такой, что $\pi_{1}\left(S_{F}\right)=F$. В случае тривиальной подгруппы $F=[1]$ накрывающая поверхность $\widetilde{S} \equiv S_{1}$ называется универсальным накрытием поверхности $S$. Поверхность $\widetilde{S}$ односвязна, и по теореме униформизации Кёбе и Пуанкаре существует конформный гомеоморфизм $h$ этой поверхности на одну из канонических областей $\mathbb{D}=\{z \in \mathbb{C}:|z|<$ $1\}, \mathbb{C}$ или $\widehat{\mathbb{C}}$. Соответственно поверхность $S$ в этом случае называется гиперболической, параболической или эллиптической. В частности, гиперболическая односвязная область обязательно имеет более двух граничных точек. Послойный гомеоморфизм поверхности $\widetilde{S}$ порождает конформный автоморфизм канонической области, а композиция $J \circ h^{-1}$ с проекцией $J: \widetilde{S} \rightarrow S$ является автоморфной аналитической функцией относительно группы автоморфизмов этой области. Поэтому универсальное накрытие можно отождествить с его образом относительно $h$. Обозначим через $G$ группу автоморфизмов канонической области, отвечающую группе послойных преобразований поверхности $\widetilde{S}$. Тогда любую риманову поверхность $S$ можно отождествить с фактором $S=D / G$, где $D$ - каноническая область, с точностью до конформной эквивалентности. В этом случае говорят, что группа $G$ униформизует поверхность $S$.

Любая эллиптическая риманова поверхность конформно эквивалентна римановой сфере $\widehat{\mathbb{C}}$. Группа $G$ в этом случае состоит из дробно-линейных конформных автоморфизмов римановой сферы $\widehat{\mathbb{C}}$. Любая параболическая риманова поверхность конформно эквивалентна либо плоскости $\mathbb{C}$, либо проколотой плоскости $\mathbb{C} \backslash\{0\}$, либо тору. Группа $G$ в этом случае либо является тривиальной, либо состоит из преобразований вида $z^{\prime}=z+n \omega$, либо состоит из преобразований вида $z^{\prime}=z+n \omega_{1}+m \omega_{2}$, где $\omega, \omega_{1}, \omega_{2}-$ комплексные числа c $\operatorname{Im}\left(\omega_{2} / \omega_{1}\right) \neq 0, n, m$ - целые числа. Все другие римановы поверхности имеют гиперболический тип, и группа $G$ является при этом дискретной подгруппой группы Мёбиуса Möb всех конформных автоморфизмов круга $\mathbb{D}$. Напомним, что указанные автоморфизмы задаются формулой

$$
z \mapsto e^{i \theta} \frac{z-a}{1-z \bar{a}}, \quad a \in \mathbb{D}, \quad 0 \leqslant \theta<2 \pi
$$

В этом случае группа $G$ называется фуксовой. Фундаментальная область $D$ фуксовой группы $G$ является фактором $\mathbb{D}$, состоящим из точек, которые нельзя перевести действием $G$ друг в друга. Геометрически, указанная область является гиперболическим многоугольником в круге $\mathbb{D}$, ограниченным дугами орициклов и кусками границы $\partial \mathbb{D}$. Если этот многоугольник имеет конечное число сторон, то группа $G$ конечно порождена. Риманова поверхность $S$ с конечно порожденной униформизующей группой $G$ называется иначе конечной римановой поверхностъю. Если она имеет род $g$, то в группе $G$ можно выбрать образующие $A_{1}, B_{1}, \ldots, A_{g}, B_{g}$ с неподвижными гиперболическими точками на границе $\partial \mathbb{D}$, отвечающие сторонам многоугольника $D$. Если, кроме того, на поверхности имеются $n$ проколов и $r$ точек ветвления, то можно добавить к ним 
образующие $C_{1}, \ldots, C_{n+r}$, отвечающие $n$ фиксированным параболическим точкам преобразований $C_{1}, \ldots, C_{n}$ (каспы на границе $\left.\partial D\right)$ и $r$ эллиптическим точкам преобразований $C_{n+1}, \ldots, C_{n+r}$ (вершины $D$, лежащие в $\left.\mathbb{D}\right)$. Эти образующие удовлетворяют единственному соотношению

$$
C_{n+r} \circ \cdots \circ C_{1} \circ B_{g}^{-1} \circ A_{g}^{-1} \circ B_{g} \circ A_{g} \circ \cdots \circ B_{1}^{-1} \circ A_{1}^{-1} \circ B_{1} \circ A_{1}=\text { id . }
$$

Если поверхность $S$ имеет, кроме того, $l$ гиперболических граничных компонент, то говорят, что это поверхность конечного типа $(g, n, l)$.

Итак, риманова поверхность $S$ имеет конечный тun $(g, n)$, если $S=\widehat{S} \backslash F$, где $\widehat{S}$ - компактная риманова поверхность рода $g$, а $F$ - конечный набор из $n$ точек. Окрестности граничных точек поверхности $S$ представляют собой проколотые диски. Римановыми поверхностями $S$ конечного типа $(g, n, m)$ мы называем поверхности вида $S=S^{\prime} \backslash E$, где $S^{\prime}$ имеет конечный тип $(g, n)$, а $E$ конечный набор из $m$ гиперболических дисков, вложенных в $S^{\prime}$.

2.2. Квазиконформные отображения. Квазиконформные отображения были введены Гербертом Грёчем (1902-1993) в работе [38] 1928 г. и заново определены Ларсом Альфорсом [6] в 1935 г. как гомеоморфизмы плоских областей, отображающие в первом порядке малые окружности в малые эллипсы с равномерно ограниченными эксцентриситетами. Отметим, что филдсовская медаль была присуждена Альфорсу именно за его исследования по теории квазиконформных отображений. Квазиконформные отображения размерности 3 были введены Михаилом Алексеевичем Лаврентьевым (1900-1980) в работах [64], [65] 1938 г., где изучались решения эллиптических уравнений.

Начнем с определения квазиконформных отображений плоских областей. Пусть $D$ - область в $\widehat{\mathbb{C}}$ (возможно, совпадающая с $\widehat{\mathbb{C}})$ и $w=f(z)$ - гомеоморфизм $D$ на другую область $D^{\prime} \subseteq \widehat{\mathbb{C}}$. Определим производные

$$
f_{\bar{z}}:=\frac{\partial f}{\partial \bar{z}}=\frac{1}{2}\left(\frac{\partial f}{\partial x}+i \frac{\partial f}{\partial y}\right), \quad f_{z}:=\frac{\partial f}{\partial z}=\frac{1}{2}\left(\frac{\partial f}{\partial x}-i \frac{\partial f}{\partial y}\right), \quad z=x+i y,
$$

в смысле обобщенных функций, локально суммируемые с квадратом на $D$. Гомеоморфизм $f$ называется квазиконформнъм в области $D$, если комплекснозначная функция $\mu_{f}(z)=f_{\bar{z}} / f_{z}$ удовлетворяет неравенству $\left|\mu_{f}(z)\right| \leqslant k<1$ почти всюду в $D$. Гомеоморфизм $f$ при этом называется $K$-квазиконформным, а $K$ задается соотношением $K=(1+k) /(1-k)$. Функция $\mu_{f}(z)$ называется комплексной характеристикой или комплексной дилатацией отображения $f$. Заметим, что квазиконформное отображение конформно тогда и только тогда, когда $K=1$ (или $k=0)$.

Для дальнейшего удобно ввести следующие обозначения. В случае римановой сферы обозначим через $\widehat{\mathbb{C}}_{K}$ класс всех $K$-квазиконформных автоморфизмов $f: \widehat{\mathbb{C}} \rightarrow \widehat{\mathbb{C}}$, нормированных условиями $f(0)=0, f(1)=1, f(\infty)=\infty$. В случае единичного круга обозначим через $\mathbb{D}_{K}$ класс всех $K$-квазиконформных автоморфизмов $f: \mathbb{D} \rightarrow \mathbb{D}$ с нормировкой $f( \pm 1)= \pm 1, f(i)=i$.

Квазиконформное отображение является гомеоморфным обобщенным решением уравнения Бельтрами

$$
w_{\bar{z}}=\mu_{f}(z) w_{z} .
$$

Это решение определено единственным образом с точностью до конформных гомеоморфизмов. Добавляя какое-либо из условий конформной нормировки 
(например, фиксируя три неподвижные точки на границе в случае односвязной области), мы можем добиться единственности решения уравнения (1).

Переходя к геометрическому определению квазиконформных отображений, воспользуемся понятием модуля семейства кривых. В терминах этого понятия сохраняющий ориентацию гомеоморфизм $f$ области $D$ на область $D^{\prime}$ называется $K$-квазиконформным, если для любой двусвязной гиперболической области $R \subset D$ отношение модулей $M(f(R)) / M(R)$ ограничено и выполняется следующее неравенство:

$$
\frac{M(R)}{K} \leqslant M(f(R)) \leqslant K M(R)
$$

где $M(R)$ - модуль семейства кривых, разделяющих компоненты границы $\partial R$. Неравенство (2) иначе называется квазиинвариантностью модуля.

Рассмотрим подробнее связь между квазиконформным отображением $f$ и его дилатацией $\mu_{f}(z)$. Дилатация $\mu_{f}(z)$ является ограниченной измеримой функцией от $z$ с нормой $\left\|\mu_{f}\right\|_{\infty}<1$. Если $\mu_{f}(z)$ есть $n$-кратно дифференцируемая функция от $z$ с $n \geqslant 1, n$-я производная которой непрерывна по Гёльдеру с показателем $\alpha \in(0,1)$, то квазиконформное решение $f^{\mu} \in \mathbb{D}_{K}$ уравнения (1) является $(n+1)$-кратно дифференцируемым, а его $(n+1)$-я производная удовлетворяет условию Гёльдера с тем же показателем [13]. Можно было бы ожидать, что $f^{\mu}$ непрерывно дифференцируема для любой непрерывной функции $\mu$. Однако это неверно, как показывает пример отображения $f(z)=z(1-\log |z|)$, $f(0)=0, z \in \mathbb{D}$, которое не является даже непрерывным по Липшицу в точке $z=0$. П. П. Белинский в работе [13] показал, что непрерывной функции $\mu$ отвечает решение $f$, которое непрерывно по Гёльдеру с любым показателем $0<\alpha<1$.

Предположим, что дилатация $\mu_{f}(z, t)$ квазиконформного гомеоморфизма $f$ в единичном круге $\mathbb{D}$ зависит от вещественного или комплексного параметра $t$. Тогда если функция $\mu(\cdot, t)$ является непрерывной, дифференцируемой или голоморфной функцией параметра $t$, то таким же свойством обладает и решение $f^{\mu}$.

Обратимся к понятию квазиконформности отображений римановых поверхностей. Пусть $S_{0}=\mathbb{D} / G_{0}$ и $\omega-$ квазиконформный автоморфизм круга $\mathbb{D}$, совместимый с действием фуксовой группы $G_{0}$. Обозначим через $m_{\omega}$ дилатацию отображения $\omega$. Тогда

$$
m_{\omega}(\gamma(z)) \frac{\overline{\gamma^{\prime}(z)}}{\gamma^{\prime}(z)}=m_{\omega}(z) \quad \text { для } \gamma \in G_{0} .
$$

Введем фуксову группу $G=\omega \circ G_{0} \circ \omega^{-1}$ и обозначим через $S$ риманову поверхность $S=\mathbb{D} / G$. Имеет место следующая коммутативная диаграмма:

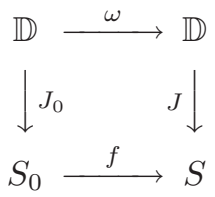


в которой $f=J \circ \omega \circ J_{0}^{-1}$ есть квазиконформный гомеоморфизм $S_{0}$ на $S$, а $J$ и $J_{0}$ - автоморфные проекции. Дилатация отображения $f$ равна

$$
\mu_{f}(\zeta)=m_{\omega}\left(J_{0}^{-1}(\zeta)\right) \frac{\overline{\left(J_{0}^{-1}(\zeta)\right)^{\prime}}}{\left(J_{0}^{-1}(\zeta)\right)^{\prime}}
$$

в терминах локального параметра $\zeta$. Фуксовы группы $G_{0}$ и $G$ являются группами автоморфности функций $J_{0}$ и $J$ соответственно, и гомеоморфизм $f$ индуцирует изоморфизм $\chi_{f}: G_{0} \rightarrow G$ этих групп. При изменении $\omega$ дилатации $m_{\omega}$ заполняют весь единичный шар $D\left(G_{0}\right)$ в пространстве $B\left(G_{0}\right)$ дифференциалов Бельтрами с конечной нормой $\|\cdot\|_{\infty}$, инвариантных относительно действия группы $G_{0}$.

Голоморфный (соответственно мероморфный) квадратичный дифберенииaл есть голоморфная (соответственно мероморфная) симметрическая 2-форма на римановой поверхности $S$. Каждой точке $p$ на поверхности $S$ она ставит в соответствие $(0,2)$-тензор $\varphi=\varphi(z) d z^{2}$, где $z$ - локальная координата в этой точке. Функция $\varphi(z)$ удовлетворяет условию инвариантности относительно замены переменной, иначе говоря, если $\varphi^{*}$ и $\varphi$ - два представления одного и того же дифференциала в терминах локальных параметров $\zeta^{*}$ и $\zeta$, то

$$
\varphi^{*}\left(\zeta^{*}\right)=\varphi(\zeta)\left(\frac{d \zeta}{d \zeta^{*}}\right)^{2}
$$

где замена $\zeta=\zeta\left(\zeta^{*}\right)$ определена на пересечении соответствующих координатных окрестностей.

Обозначим через $H^{2,0}\left(S_{0}\right)$ пространство голоморфных квадратичных дифференциалов на поверхности $S_{0}$ типа $(g, n)$ с конечной $L^{1}$-нормой. Такой дифференциал, в частности, может иметь не более чем простые полюсы на компактификациии $\widehat{S}_{0}$ поверхности $S_{0}$. Поэтому по теореме Римана-Роха

$$
\operatorname{dim}_{\mathbb{C}} H^{2,0}\left(S_{0}\right)=\left\{\begin{array}{lll}
n-3, & g=0, \\
1, & g=1, \quad n=0, \\
n-1, & g=1, \quad n>0, \\
3 g-3+n, & g \geqslant 2 .
\end{array}\right.
$$

Введем конформно инвариантную метрику, порождаемую квадратичным дифференциалом $\varphi$. Элемент длины в этой метрике равен $|d w|=\sqrt{|\varphi(\zeta)|}|d \zeta|$. Вычисляя квадратичный дифференциал $\varphi$ вдоль заданной кривой $\gamma=\gamma(t)$, получим функцию от $t$, которую естественно отождествлять с ограничением $\left.\varphi\right|_{\gamma}$ дифференциала $\varphi$ на кривую $\gamma$. Будем называть траекторией дифференuиала $\varphi$ на $S_{0}$ максимальную регулярную кривую $\gamma$, ограничение $\varphi$ на которую положительно: $\left.\varphi\right|_{\gamma}>0$; траектория называется ортогональной, если ограничение $\varphi$ на нее удовлетворяет обратному неравенству: $\left.\varphi\right|_{\gamma}<0$. Траектории и ортогональные траектории квадратичных дифференциалов можно определить в терминах внутренней дифференциальной геометрии поверхности, поэтому они не зависят от выбора локального параметра. Мы называем траекторию (соответственно ортогональную траекторию) критической, если в ее замыкании содержится нуль или полюс функции $\varphi$; в противном случае траектория называется регулярной. Подробное описание локальной и глобальной структуры траекторий можно найти, например, в работах [48], [103], [118]. 
Отображения Тейхмюллера играют важнейшую роль в теории квазиконформных отображений. Они являются обобщенными $K$-квазиконформными гомеоморфными решениями $f$ уравнения Бельтрами (1) с комплексной дилатацией

$$
\mu_{f}(\zeta)=k \frac{\overline{\varphi(\zeta)}}{|\varphi(\zeta)|}, \quad k=\frac{K-1}{K+1},
$$

где $\varphi(\zeta) d \zeta^{2}$ - голоморфный квадратичный дифференциал на $S_{0}$. Обратное отображение $f^{-1}$ к отображению Тейхмюллера $f$ также является отображением Тейхмюллера, т. е. существует голоморфный квадратичный дифференциал $\psi(w) d w^{2}$ на $f\left(S_{0}\right)$ такой, что обратное отображение имеет дилатацию $\mu_{f^{-1}}(w)=k \overline{\psi(w)} /|\psi(w)|$. Отображения Тейхмюллера локально аффинны и отображают инфинитезимальные окружности в инфинитезимальные эллипсы, большие полуоси которых направлены вдоль траекторий дифференциала $\psi(w) d w^{2}$ или перпендикулярно к ним. Отношение большой полуоси эллипса к его малой полуоси равно $K$ в каждой точке $S_{0}$, не являющейся особой для $\varphi$. Другими словами, существует квадратичный дифференциал $\varphi(\zeta) d \zeta^{2}$ на $S_{0}$ такой, что вне его нулей можно построить карты на поверхностях $S_{0}$ и $S$, в которых $\varphi(\zeta) d \zeta^{2}=d z^{2}$ и $f(x+i y)=K x+i y$.

2.3. Определение пространства Тейхмюллера, его касательное и кокасательное пространства. В определении пространства Тейхмюллера конечных римановых поверхностей мы следуем оригинальному подходу Тейхмюллера.

Пусть заданы неотрицательные целые числа $g, n$ и риманова поверхность $S_{0}$ конечного типа $(g, n)$, т. е. поверхность рода $g$ с $n$ проколами. Если $3 g-3+n>0$, то $S_{0}$ имеет гиперболический конформный тип и ее универсальное накрытие конформно эквивалентно единичному кругу $\mathbb{D}$. Послойные преобразования, меняющие местами листы универсального накрытия, индуцируют отображения из соответствующей фуксовой группы $G_{0}$ автоморфизмов Мёбиуса круга $\mathbb{D}$, которая униформизует риманову поверхность $S_{0}: S_{0}=\mathbb{D} / G_{0}$.

Пространство Тейхмюллера $T\left(S_{0}\right)$ есть пространство аналитических структур на конечной римановой поверхности $S_{0}$, при этом две структуры считаются эквивалентными, если между ними существует конформное отображение, гомотопное тождественному. Иными словами, это фактор пространства оснащенных (по отношению к исходной поверхности $S_{0}$ ) римановых поверхностей заданного (аналитического конечного) конформного типа. Более подробно, пусть $S$ есть риманова поверхность и $f$ - гомеоморфизм исходной римановой поверхности $S_{0}$ на $S$. Рассмотрим пару $(S,[f])$ как оснащенную риманову поверхность, где $[f]$ есть класс всех гомеоморфизмов $S_{0} \rightarrow S$, гомотопных $f$. Через $(S, f)$ будем обозначать оснащенную риманову поверхность, для которой $f$ является одним из представителей класса $[f]$. Оснащенные римановы поверхности $\left(S_{1}, f_{1}\right)$ и $\left(S_{2}, f_{2}\right)$ называются эквивалентными, если существует конформный гомеоморфизм $h: S_{1} \rightarrow S_{2}$ такой, что отображение $f_{2}^{-1} \circ h \circ f_{1}$ гомотопно тождественному отображению на $S_{0}$. Класс эквивалентности оснащенных римановых поверхностей задает точку пространства Тейхмюллера, а множество всех классов эквивалентности образует пространство Тейхмюлле$p a T \equiv T\left(S_{0}\right)$ (относительно исходной поверхности $\left.S_{0}\right)$. Класс эквивалентности, 
содержащий оснащенную риманову поверхность $\left(S_{0}, \mathrm{id}\right)$, считается начальной точкой 0 пространства $T\left(S_{0}\right)$. Пространство Тейхмюллера $T\left(S_{0}\right)$ является комплексным аналитическим многообразием комплексной размерности $3 g-3+n$.

Его можно также определить как множество классов эквивалентности комплексных структур. Пусть $\varphi: U \rightarrow \mathbb{C}$ и $\psi: V \rightarrow \mathbb{C}$ - максимальные атласы, отвечающие двум комплексным структурам на $S_{0}$. Они называются эквивалентными, если существует гомеоморфизм $f: S_{0} \rightarrow S_{0}$, изотопный тождественному, такой, что $\varphi \circ f=\psi$. Тогда пространство $T\left(S_{0}\right)$ можно отождествить с множеством классов эквивалентности всех комплексных структур на $S_{0}$.

Пространство Тейхмюллера $T\left(S_{0}\right)$ является связным и односвязным метрическим пространством, наделенным естественной метрикой Тейхмюллера $\tau_{T}(x, y)$. Предположим, что $\left(S_{1}, f_{1}\right)$ и $\left(S_{2}, f_{2}\right)$ - две оснащенные римановы поверхности. Гомеоморфизм $f_{2} \circ f_{1}^{-1}: S_{1} \rightarrow S_{2}$ порождает гомеоморфизм оснащенных римановых поверхностей. Среди всех гомеоморфизмов, гомотопных $f_{2} \circ f_{1}^{-1}$, обязательно имеются квазиконформные отображения (см., например, [16]). Пусть оснащенная поверхность $\left(S_{1}, f_{1}\right)$ отвечает точке $x \in T\left(S_{0}\right)$ пространства Тейхмюллера, а поверхность $\left(S_{2}, f_{2}\right)$ - точке $y \in T\left(S_{0}\right)$. Тогда

$$
\tau_{T}(x, y):=\frac{1}{2} \inf _{f} \log \frac{1+\left\|\mu_{f}\right\|_{\infty}}{1-\left\|\mu_{f}\right\|_{\infty}},
$$

где $f$ пробегает все квазиконформные гомеоморфизмы, гомотопные $f_{2} \circ f_{1}^{-1}$, $\left\|\mu_{f}\right\|_{\infty}=\operatorname{ess}_{\sup _{z \in S_{1}}}\left|\mu_{f}(z)\right|<1$.

Пусть $S_{0}=\mathbb{D} / G_{0}$ и $B\left(G_{0}\right)$ обозначает пространство дифференциалов Бельтрами $\mu=\mu(z) d \bar{z} / d z$, инвариантных относительно действия группы $G_{0}$, т. е. $\mu(z)$ есть измеримая функция в круге $\mathbb{D}$ с $\|\mu\|_{\infty}<\infty$ и

$$
\mu(\gamma(z)) \frac{\overline{\gamma^{\prime}(z)}}{\gamma^{\prime}(z)}=\mu(z)
$$

для любого $\gamma \in G_{0}$. Обозначим через $D\left(G_{0}\right)$ единичный шар в пространстве $B\left(G_{0}\right): D\left(G_{0}\right)=\left\{\mu \in B\left(G_{0}\right):\|\mu\|_{\infty}<1\right\}$. Каждый дифференциал $\mu \in$ $D\left(G_{0}\right)$ задает посредством решения уравнения Бельтрами единственный квазиконформный автоморфизм $\omega^{\mu}: \mathbb{D} \rightarrow \mathbb{D}$ единичного круга, удовлетворяющий условию нормировки: $\omega^{\mu}( \pm 1)= \pm 1, \omega^{\mu}(i)=i$. Обозначим через $I\left(G_{0}\right)$ nространство тривиальных дифференииалов Бельтрами, состоящее из дифференциалов $\mu \in D\left(G_{0}\right)$, для которых отображение $\omega^{\mu}$ действует тождественно на $\partial \mathbb{D}$. Пространство Тейхмюллера $T\left(S_{0}\right)$ можно отождествить с фактором $T\left(G_{0}\right)=D\left(G_{0}\right) / I\left(G_{0}\right)$, тем самым, у нас имеется естественная голоморфная проекция $\Phi: D\left(G_{0}\right) \rightarrow T\left(G_{0}\right)$.

Римановы поверхности одинакового конформного типа $(g, n)$ порождают изоморфные пространства Тейхмюллера, поэтому можно считать, что имеется единственное пространство Тейхмюллера $T(g, n)$. Однако иногда удобнее рассматривать пространство Тейхмюллера с фиксированной начальной римановой поверхностью $S_{0}$.

Обозначим через $H^{2,0}\left(G_{0}\right)$ пространство всех квадратичных голоморфных дифференциалов $q=q(z) d z^{2}$ в круге $\mathbb{D}$, инвариантных относительно группы $G_{0}: q(\gamma(z))\left(\gamma^{\prime}(z)\right)^{2}=q(z), \gamma \in G_{0}$. Иначе говоря, $H^{2,0}\left(G_{0}\right)$ есть пространство всех параболических 2-форм веса $(-4)$ относительно группы $G_{0}$ с конечной 
нормой

$$
\|q\|:=\sup _{\mathbb{D}}\left(1-|z|^{2}\right)^{2}|q(z)|<\infty .
$$

Проекция $H^{2,0}\left(G_{0}\right)$ на $S_{0}$ совпадает с пространством квадратичных голоморфных дифференциалов $\varphi$ на $S_{0}$, которые в случае конечных римановых поверхностей имеют конечную интегральную норму

$$
\iint_{S_{0}}|\varphi(\zeta)| d \sigma_{\zeta}<\infty
$$

На пространстве $B\left(G_{0}\right) \times H^{2,0}\left(G_{0}\right)$ можно ввести естественное спаривание

$$
A(\mu)=\iint_{\mathbb{D} / G_{0}} \mu q d \sigma_{z}
$$

Обозначим через $N\left(G_{0}\right)$ пространство локально тривиальнъх дифференииалов Бельтрами, т. е. подпространство $B\left(G_{0}\right)$, совпадающее с ядром оператора $A(\mu)$. Тогда касательное пространство к $T\left(G_{0}\right)$ в начальной точке можно отождествить с пространством $H\left(G_{0}\right):=B\left(G_{0}\right) / N\left(G_{0}\right)$.

Сопоставим ему некоторое подпространство в $B\left(G_{0}\right)$ следующим образом. Определим интеграл Бергмана $\Lambda_{\mu}: \mu \mapsto \Lambda_{\mu}(z) \in H^{2,0}\left(G_{0}\right)$ по формуле

$$
\Lambda_{\mu}=\frac{12}{\pi} \iint_{\mathbb{D} / G_{0}} \frac{\overline{\mu(\zeta)}}{(\zeta-z)^{4}} d \sigma_{\zeta}, \quad \mu \in B\left(G_{0}\right) .
$$

Пространство $N\left(G_{0}\right)$ совпадает с ядром оператора $\Lambda_{\mu}$. Обозначим через $\Lambda_{q}^{*}$ : $q \mapsto \Lambda_{q}^{*}(z) \in B\left(G_{0}\right)$ двойственный оператор, задаваемый формулой

$$
\Lambda_{q}^{*}(z)=\overline{q(z)}\left(1-|z|^{2}\right)^{2}, \quad q \in H^{2,0}\left(G_{0}\right) .
$$

Композиция $\Lambda_{\mu} \circ \Lambda_{q}^{*}$ является тождественным оператором на пространстве $H^{2,0}\left(G_{0}\right)$, тем самым, оператор $\Lambda_{q}^{*}$ расщепляет точную последовательность

$$
0 \rightarrow N\left(G_{0}\right) \hookrightarrow B\left(G_{0}\right) \stackrel{\Lambda_{\mu}}{\rightarrow} H^{2,0}\left(G_{0}\right) \rightarrow 0 .
$$

Поэтому $H\left(G_{0}\right)=\Lambda_{q}^{*}\left(H^{2,0}\left(G_{0}\right)\right) \cong B\left(G_{0}\right) / N\left(G_{0}\right)$. Касательное пространство $H(G)$ в любой другой точке $T\left(G_{0}\right)$ можно получить, применяя те же рассуждения к группе $G=\chi_{\mu}\left(G_{0}\right)$, где $\chi_{\mu}$ есть групповой изоморфизм, задаваемый отображением $\chi_{\mu}: G_{0} \mapsto \omega^{\mu} \circ G_{0} \circ\left(\omega^{\mu}\right)^{-1}$. Спаривание $\langle\mu, q\rangle:=A(\mu)$ определяет кокасательное пространство $H^{2,0}\left(G_{0}\right)$, на котором имеется эрмитово произведение Петерсона, задаваемое формулой

$$
\left(q_{1}, q_{2}\right)=\iint_{\mathbb{D} / G_{0}} q_{1}(z) \overline{q_{2}(z)}\left(1-|z|^{2}\right)^{2} d \sigma_{z} .
$$

На касательном пространстве $T\left(G_{0}\right)$ можно ввести (см., например, [124]) кэлерову метрику Вейля-Петерсона $\left\{\mu_{1}, \mu_{2}\right\}=\left\langle\mu_{1}, \Lambda_{\mu_{2}}\right\rangle$, тем самым наделяя пространство $T\left(G_{0}\right)$ структурой кэлерова многообразия. 
2.4. Теорема Тейхмюллера. Как уже отмечалось, вещественная дилатация

$$
K(f)=\frac{1+\left\|\mu_{f}\right\|_{\infty}}{1-\left\|\mu_{f}\right\|_{\infty}}
$$

является мерой отклонения квазиконформного отображения $f$ от конформного. Пусть $(S, f)$ есть оснащенная (относительно $S_{0}$ ) риманова поверхность и $f$ - гомеоморфизм $S_{0} \rightarrow S$, являющийся представителем гомотопического класса $[f]$ гомеоморфизмов $S_{0}$ на $S$. Любой гомеоморфизм $g: S \rightarrow S^{\prime}$ порождает гомеоморфизм оснащенных римановых поверхностей $(S, f) \rightarrow\left(S^{\prime}, f^{\prime}\right)$, где $f^{\prime}=g \circ f$. Пусть $(S, f)$ и $\left(S^{\prime}, f^{\prime}\right)$ - две римановы поверхности типа $(g, n, l)$. Тогда, как было отмечено выше, любой гомотопический класс гомеоморфизмов $(S, f)$ на $\left(S^{\prime}, f^{\prime}\right)$ обязательно содержит квазиконформные отображения.

Следующий фундаментальный результат теории квазиконформных отображений был сформулирован Тейхмюллером в работах [108], [109] и строго доказан Альфорсом и Берсом в [11]. Другое доказательство этого факта было предложено Крушкалем [58]. Разобьем его для удобства на два отдельных предложения.

ПредЛОжЕНИЕ 1. Пусть $f: S_{1} \rightarrow S_{2}$ есть отображсение Тейхмюллера между двумя оснащенными (относительно $S_{0}$ ) гиперболическими поверхностями $S_{1}$ и $S_{2}$. Тогда $f$ есть единственное экстремальное квазиконформное отображение в своем гомотопическом классе $[f]$. Иначе говоря, $f$ либо конформно (т.е. $K(f)=1)$, либо $K(g)>K(f)$ для любого квазиконформного отображения $g$, гомотопного $f$ и такого, что $f \neq g$ почти всюду на $S_{1}$. В частности, $\tau_{T}\left(S_{1}, S_{2}\right)=(1 / 2) \log K(f)$.

Следующее предложение часто называют теоремой Тейхмюллера.

ПреДЛОЖенИЕ 2. Пусть $S_{1}$ и $S_{2}$ - две оснащенные (относителъно $S_{0}$ ) гиперболические поверхности из пространства $T\left(S_{0}\right)$. Тогда существует единственное отображение Тейхмюллера $f: S_{1} \rightarrow S_{2}$.

В частности, два различных отображения Тейхмюллера никогда не гомотопны друг другу.

Риманова сфера $\widehat{\mathbb{C}}$ обладает единственной комплексной структурой, поэтому ее пространство Тейхмюллера тривиально (состоит из одной точки). Пространство Тейхмюллера тора $\mathbb{T}$ изоморфно верхней полуплоскости $\mathbb{H}=\{z \in$ $\mathbb{C}: \operatorname{Im} z>0\}$. Конформная метрика на нем есть гиперболическая метрика вида $|d z| /(2 \operatorname{Im} z)$ с постоянной кривизной, равной -4 . Пространство Тейхмюллера $T(0,4)$ изометрично $T(1)$ и $T(1,1)$, поэтому метрика Тейхмюллера в этом случае гиперболична. Теоремы Тейхмюллера выполняются и в этом случае, т. е. отображение Тейхмюллера существует и единственно с точностью до конформных автоморфизмов тора $\mathbb{T}$, изотопных тождественному.

Отображения Тейхмюллера позволяют идентифицировать вещественные и комплексные геодезические в метрике Тейхмюллера. А именно, рассмотрим отображение Тейхмюллера $f^{\mu}$ с дифференциалом Бельтрами

$$
\mu(z)=\overline{\varphi(z)} /|\varphi(z)|
$$


на $S_{0}$, сопоставляющее $t \in \mathbb{D}$ точку $T\left(S_{0}\right)$. Тогда отображение $f^{\mu}:(-1,1) \rightarrow$ $T\left(S_{0}\right)$ задает вещественную геодезическую в метрике Тейхмюллера. С другой стороны, голоморфное вложение $f^{\mu}: \mathbb{D} \rightarrow T\left(S_{0}\right)$ определяет комплексный геодезический диск в пространстве $T\left(S_{0}\right)$.

2.5. Инвариантные метрики. Пространство Тейхмюллера $T(g, n)$ является $(3 g-3+n)$-мерным гиперболическим комплексным аналитическим многообразием, на котором можно вводить биголоморфно инвариантные метрики, обобщающие гиперболическую метрику Пуанкаре. Нас особенно интересуют метрики Кобаяси $k_{T}(x, y)$ и Каратеодори $c_{T}(x, y), x, y \in T(g, n)$. Их определение и свойства можно найти в книгах [32], [62], [87].

Пусть $S_{0}=\mathbb{D} / G_{0}$ и $q=q(z) d z^{2}$ - голоморфный квадратичный дифференциал в круге $\mathbb{D}$, удовлетворяющий условию инвариантности: $q(\gamma(z))\left(\gamma^{\prime}(z)\right)^{2}=q(z)$ для любого $\gamma \in G_{0}$. Будем называть диском Тейхмюллера комплексный геодезический диск в метрике Тейхмюллера, иными словами, голоморфное вложение единичного диска в пространство Тейхмюллера $T\left(S_{0}\right)$ в виде $\Delta_{q}:=$ $\{\Phi(t \bar{q} /|q|), t \in \mathbb{D}\}$, где $\Phi: D\left(G_{0}\right) \rightarrow T\left(G_{0}\right)$ - естественная голоморфная проекция.

Обозначим через $d(\cdot, \cdot)$ обычную гиперболическую метрику Пуанкаре в единичном круге $\mathbb{D}$ :

$$
d\left(z_{1}, z_{2}\right)=\frac{1}{2} \log \frac{1+\left|\left(z_{1}-z_{2}\right) /\left(1-z_{1} \bar{z}_{2}\right)\right|}{1-\left|\left(z_{1}-z_{2}\right) /\left(1-z_{1} \bar{z}_{2}\right)\right|} .
$$

Пусть $X, A, B$ - комплексно аналитические многообразия и $\operatorname{Hol}(A, B)$ обозначает класс всех голоморфных отображений $A \rightarrow B$. Полуметрика Кобаяси $k_{X}(x, y)$ на $X$ есть наибольшая из полуметрик $\rho$ на $X$, удовлетворяющих неравенству $\rho\left(f\left(z_{1}\right), f\left(z_{2}\right)\right) \leqslant d\left(z_{1}, z_{2}\right)$ для любого $f \in \operatorname{Hol}(\mathbb{D}, X) ; z_{1}, z_{2} \in \mathbb{D}$. Полуметрика Каратеодори $c_{X}(x, y)$ есть наименьшая из полуметрик $\rho$ на $X$, удовлетворяющих неравенству $\rho(x, y) \geqslant d(f(x), f(y))$ для любого $f \in \operatorname{Hol}(X, \mathbb{D})$; $x, y \in X$. Обе полуметрики на $X$ обладают главными свойствами гиперболической метрики Пуанкаре: свойством уменьшения метрики при голоморфных отображениях (аналогичным лемме Шварца) и свойством биголоморфной инвариантности. В частности, если $X$ есть подмногообразие в $T(g, n)$, то $c_{X}(x, y) \geqslant c_{T}(x, y)$. На самом пространстве Тейхмюллера и его подмногообразиях обе полуметрики являются настоящими метриками. Метрика Кобаяси к тому же является внутренней, т. е. может быть восстановлена по своей инфинитезимальной метрике, тогда как метрика Каратеодори этим свойством не обладает. По-видимому, в этом и состоит главное различие между двумя указанными метриками. Заметим, что всегда $k_{X}(x, y) \geqslant c_{X}(x, y)$.

Метрика Кобаяси на пространстве $T(g, 0)$ была полностью описана Ройденом [94] в 1971 г. Эрл и Кра (см. [27], [20]) в 1974 г. перенесли эти результаты на пространство $T(g, n)$. Они показали, что $k_{T}(x, y)=\tau_{T}(x, y)$. Для пространства Тейхмюллера $T(0,4)$ метрики $k_{T}(x, y)$ и $c_{T}(x, y)$ совпадают, поскольку это пространство биголоморфно эквивалентно гиперболической области в $\mathbb{C}$. В [20] Абикоф, Эрл и Кра сформулировали гипотезу о том, что указанные метрики должны совпадать на любом пространстве Тейхмюллера. Однако в 1981 г. Крушкаль (см. [59], [61], а также [62]) построил пример, показывающий, что при выполнении условия $3 g-3+n>2, n \geqslant 1, g>2$, для любой точки $x$ 
на пространстве Тейхмюллера $T(g, n)$ найдется точка $y \in T(g, n)$ такая, что $k_{X}(x, y)>c_{X}(x, y)$. Тем не менее в том же году Кра [56] удалось доказать и некоторое утверждение в положительном направлении. А именно, он показал, что на дисках Тейхмюллера $\Delta_{q}$ таких, что $q$ есть квадрат абелева голоморфного дифференциала на $S_{0}$, обе метрики совпадают. После этого появился целый ряд утверждений в противоположном направлении. В частности, было показано, что вложение Берса в пространство $T(g, n)$ (см. [11]) не является геодезическим в метрике Каратеодори (Наг [70]). Затем аналогичный результат был получен Крушкалем [60] для универсального пространства Тейхмюллера и общего бесконечномерного пространства Тейхмюллера [68]. В случае универсального пространства Тейхмюллера Шига и Танигава [101], пользуясь квазиконформной вариацией коэффициентов Грунского, построили пример неабелева диска Тейхмюллера, на котором указанные выше метрики совпадают.

2.6. Пространство модулей Римана. Пусть $S$ - гиперболическая риманова поверхность конечного типа $(g, n)$, причем $3 g-3+n>0$. Обозначим через $\operatorname{Mod}(S)$ группу изотопических классов сохраняющих ориентацию гомеоморфизмов $\phi: S \rightarrow S$, т. е. группу сохраняющих ориентацию гомеоморфизмов поверхности $S$ по модулю гомеоморфизмов, гомотопных тождественному. Группа $\operatorname{Mod}(S)$ называется модулярной группой Тейхмюллера или группой классов отображений поверхности $S$. Группа $\operatorname{Mod}(S)$ действует на пространстве $T(g, n)$ посредством отображения $\phi \cdot(S,[f])=\phi \cdot(S, f)=\left(S, f \circ \phi^{-1}\right)$. Фактор по этому действию есть пространство модулей Римана $M(g, n)=$ $T(g, n) / \operatorname{Mod}(S)$. Можно сказать, что $M(g, n)$ получается из пространства Тейхмюллера $T(g, n)$ “забыванием оснащения" поверхностей. В частности, модулярная группа Тейхмюллера тора совпадает со стандартной модулярной группой $\mathrm{SL}(2, \mathbb{Z})$.

Если $p$ - базовая точка поверхности $S$, а $\pi_{1}(S, p)$ - ее фундаментальная группа, то группа изотопических классов гомеоморфизмов $S$ естественно изоморфна группе внешних автоморфизмов $\operatorname{Aut}\left(\pi_{1}(S, p)\right) / \pi_{1}(S, p)$ группы $\pi_{1}(S, p)$. Теорема Дена-Нильсена (см. [79]) утверждает, что модулярная группа является подгруппой индекса 2 в этой группе внешних автоморфизмов, состоящей из сохраняющих ориентацию внешних автоморфизмов, которые действуют тривиально на второй группе когомологий $H^{2}\left(\pi_{1}(S, p), \mathbb{Z}\right)=H^{2}(S, \mathbb{Z})=\mathbb{Z}$.

Действие группы $\operatorname{Mod}(S)$ на $T(S)$ не является свободным. Поэтому пространство модулей является орбифолдом, в котором каждому классу изоморфизма римановых поверхностей типа, определяемого поверхностью $S$, отвечает в точности одна точка. Стабилизатор поверхности $x \in T(S)$ изоморфен группе конформных автоморфизмов этой поверхности.

Известная теорема Тёрстона [112] впервые появилась в виде препринта около 1976 г., затем была передоказана Берсом [19] в 1978 г. и наконец опубликована в 1988 г. Она дает следующее описание представителей из $\operatorname{Mod}(S)$. Любой класс отображений $[f] \in \operatorname{Mod}(S)$ имеет представителя $f$, который является либо периодическим (т. е. конечного порядка), либо приводимым, либо псевдоаносовским. Гомеоморфизм $f$ приводим, если существует множество $C$ кривых на $S$, компоненты которых остаются неподвижными под действием гомеоморфизма $f$ и только переставляются им. Отображение $f: S \rightarrow S$ называется 
псевдоаносовским, если оно является отображением Тейхмюллера с квадратичным дифференциалом $\varphi$, который сохраняется гомеоморфизмом $f$, имеет вещественную дилатацию $0<k<1$ и нормировано условием $\int_{S}|\varphi|=1$.

Теорема Тёрстона вызвала большой интерес к изучению динамики действия группы классов отображений $\operatorname{Mod}(S)$ на пространстве Тейхмюллера $T(S)$. Тёрстон построил компактификацию пространства Тейхмюллера $T(S)$, на которую естественным образом продолжается действие группы $\operatorname{Mod}(S)$. Тип элемента $g$ группы $\operatorname{Mod}(S)$ следующим образом связан с неподвижными точками этой компактификации. Если $g$ периодичен, то внутри $T(S)$ имеется неподвижная точка. Если $g$ - псевдоаносовский, то $g$ не имеет неподвижных точек на пространстве $T(S)$, однако имеет пару неподвижных точек на границе его компактификации Тёрстона. Для приводимых отображений $g$ может существовать единственная неподвижная точка на границе Тёрстона. Указанная классификация гомеоморфизмов из группы $\operatorname{Mod}(S)$ в некотором смысле аналогична делению автоморфизмов Мёбиуса единичного круга на преобразования эллиптического, параболического и гиперболического типа.

2.7. Компактификация. Существует несколько неэквивалентных способов компактификации пространства Тейхмюллера $T\left(S_{0}\right)$.

Компактификация Тейхмюллера состоит в добавлении к пространству Тейхмюллера предельных точек геодезических лучей (в метрике Тейхмюллера), исходящих из фиксированной базовой точки $S_{0}$. Эта компактификация зависит от выбора базовой точки и потому не допускает спуска на пространство модулей. Более того, Керкхоф [52] показал, что действие модулярной группы на пространстве Тейхмюллера не продолжается до непрерывного действия этой группы на указанной компактификации.

В работе [18] Берс построил компактификацию, которая совпадает с замыканием пространства $T(g)$, рассматриваемого как ограниченная область, вложенная в $\mathbb{C}^{3 g-3}$. Введем отображение $\Psi: B\left(G_{0}\right) \rightarrow H^{2,0}\left(G_{0}\right)$, которое строится следующим образом. Дифференциал Бельтрами $m(z) \in B\left(G_{0}\right)$ продолжается на всю плоскость по симметрии: $m(1 / \bar{z})=\bar{m}(z) z^{2} / \bar{z}^{2}, z \in \widehat{\mathbb{C}} \backslash \overline{\mathbb{D}}$. Положим

$$
\mu(z)= \begin{cases}0, & z \in \mathbb{D}, \\ m(z), & z \in \widehat{\mathbb{C}} \backslash \overline{\mathbb{D}} .\end{cases}
$$

Пусть $f^{\mu}$ есть решение уравнения Бельтрами $w_{\bar{z}}=\mu(z) w_{z}$, конформное в круге $\mathbb{D}$ и нормированное условием $f(0)=f^{\prime}(0)-1=0$. Согласно Нехари [76] и Краусу [57], справедлива оценка $\left\|S_{f^{\mu}}\right\| \leqslant 6$, где

$$
S_{f}=\frac{f^{\prime \prime \prime}(z)}{f^{\prime}(z)}-\frac{3}{2}\left(\frac{f^{\prime \prime}(z)}{f^{\prime}(z)}\right)^{2}, \quad z \in \mathbb{D},
$$

- производная Шварца. Заметим, что неравенство $\left\|S_{f}\right\| \leqslant 2$ гарантирует однозначность отображения $f$. Поэтому отображение $\Psi$ задает инъективное голоморфное отображение пространства Тейхмюллера $T\left(G_{0}\right)$ на открытое подмножество в $H^{2,0}\left(G_{0}\right)$. Его образ содержит шар радиуса 2 с центром в нуле и содержится в шаре радиуса 6 с тем же центром. Заметим, что действие модулярной группы не допускает непрерывного продолжения и на компактификацию Берса [53]. 
Только компактификация Тёрстона [112] совместима с действием модулярной группы. Главным инструментом для ее построения служит слоение с мерой на заданной поверхности. Слоение - это интегрируемое подрасслоение касательного расслоения. Локально оно задает разбиение многообразия на параллельные подмногообразия меньшей размерности. Слоение с мерой состоит из слоения (вообще говоря, сингулярного) и меры, сосредоточенной в трансверсальном к нему направлении. Компактификация Тёрстона совпадает с компактификацией пространства $\operatorname{PMF}\left(S_{0}\right)$ проективных слоений с мерой. Пусть $C$ есть множество изотопических классов простых замкнутых кривых на $S_{0}$ и $R^{+}(C)$ есть множество положительных функций на $C$ с топологией произведения. Обозначим через $\mathbb{P} R^{+}(C)$ соответствующее проективное пространство. Пространство $T\left(S_{0}\right)$ можно рассматривать как пространство гиперболических структур $\left(S_{0}, \rho\right)$ на $S_{0}$ по модулю следующего отношения эквивалентности: структуры $\left(S_{0}, \rho_{1}\right) \sim\left(S_{0}, \rho_{2}\right)$ эквивалентны, если существует изометрия $\left(S_{0}, \rho_{1}\right) \rightarrow\left(S_{0}, \rho_{2}\right)$, изотопная тождественному отображению. Таким образом мы можем построить отображение $\left(S_{0}, \rho_{1}\right) \rightarrow F_{\rho}$, где $F_{\rho}(\gamma) \in \mathbb{P} R^{+}(C)$ есть длина кривой $\gamma \in C$ на $\left(S_{0}, \rho\right)$. Образ пространства $T\left(S_{0}\right)$ в $\mathbb{P} R^{+}(C)$ гомеоморфен открытому шару в пространстве $\mathbb{R}^{6 g-6}$, а его граница $\operatorname{PMF}\left(S_{0}\right)$ является $(6 g-7)$-мерной сферой. Модулярная группа действует непрерывным образом на указанной компактификации Тёрстона. При этом любой ее элемент имеет на этой компактификации неподвижную точку.

Вернемся к голоморфному накрытию $P: T\left(G_{0}\right) \rightarrow M\left(G_{0}\right)$. Для пространства модулей $M\left(G_{0}\right)$ имеется известная компактификация Делиня-Мамфорда $\widehat{M}\left(G_{0}\right)$ (см. [25]), которая получается добавлением к $M\left(G_{0}\right)$ стабильных римановых поверхностей. Эти поверхности получаются из обычных римановых поверхностей "перетяжкой” (т. е. сжатием в точку простых замкнутых геодезических или дуг, соединяющих точки ветвления порядка 2 на поверхности $\left.S_{0}\right)$. Хотелось бы поднять эту компактификацию на пространство Тейхмюллера, иначе говоря, разобраться с тем, как устроено (возможно, частичное) замыкание пространства $T\left(G_{0}\right)$, отвечающее указанной компактификации пространства модулей. Такое замыкание $N T\left(G_{0}\right)$, называемое нодальным пространством Тейхмюллера (или пространством Тейхмюллера с узлами), было построено в работе [41]. Оно проецируется отображением $P$ на компактификацию Делиня-Мамфорда пространства модулей. Заметим, что конструкция из [41] годится и для клейновых групп.

Если $G_{0}$ - фуксова группа первого рода, то указанное частичное замыкание в точности совпадает с расширенным (augmented) пространством Тейхмюллера, построенным Абикофом в [1], [2]. Имеется также работа Мазура [69], который изучал пополнение метрики Вейля-Петерсона и интерпретировал граничные точки этого пополнения как нодальные римановы поверхности. Более точно, Мазур отождествил пополнение Вейля-Петерсона с расширенным пространством Абикофа, граница которого совпадает со стратифицированным объединением пространств меньшей размерности, каждое из которых является пространством Тейхмюллера нодальной поверхности. Он показал, что в некотором точно определенном смысле тангенциальная компонента метрики Вейля-Петерсона на пространстве Тейхмюллера продолжается до метрики Вейля-Петерсона на указанных расширенных пространствах Тейхмюллера.

Подробнее о компактификациях см. [46]. 
2.8. Модули семейств кривых на пространствах Тейхмюллера. Понятие модуля семейства кривых, обратное понятию экстремальной длины Альфорса и Бёрлинга, лежит в основе геометрического определения квазиконформных отображений. Оно было предложено Грёчем [38] в 1928 г. и развито далее Альфорсом и Бёрлингом в работе [12] 1946 г. С тех пор на эту тему было написано несколько обзоров и монографий, включая [26], [48], [63], [83], [102], [118].

В данном пункте с помощью модуля свободного семейства гомотопических классов кривых на римановой поверхности $S_{0}$ будет построен некоторый функционал, называемый модулем, на пространстве Тейхмюллера $T\left(S_{0}\right)$. Затем мы обсудим гармонические свойства этого функционала и дадим в его терминах описание метрики Тейхмюллера.

Обсуждаемые результаты содержатся в статьях [114], [118].

Пусть $Г$ есть семейство кривых на поверхности $S_{0}$. Обозначим через $\rho$ конформно инвариантную метрику на $S_{0}$, задаваемую локально формой $\rho(\zeta)|d \zeta|$. Иными словами, каждому локальному параметру $\zeta$ на поверхности $S_{0}$ сопоставляется измеримая вещественнозначная неотрицательная функция $\rho(\zeta)$, удовлетворяющая следующему условию инвариантности:

$$
\rho^{*}\left(\zeta^{*}\right)=\rho(\zeta)\left|\frac{d \zeta}{d \zeta^{*}}\right| .
$$

Фиксируем на римановой поверхности $S_{0}$ конечного типа $(g, n, m)$ два следующих набора кривых $\left\{\gamma_{1}, \ldots, \gamma_{m}\right\}$, которые мы будем называть кривыми типов I и II соответственно. Первое семейство (тип I) состоит из простых петель на $S_{0}$, попарно свободно негомотопных друг другу. Второе семейство (тип II) существует только при наличии у поверхности $S_{0}$ граничных гиперболических компонент (т. е. при $l>0$ ) и состоит из простых дуг на $S_{0}$ с концами на указанных компонентах. Предполагается, что все кривые гомотопически нетривиальны (т. е. негомотопны точке самой поверхности или ее замыкания) и не пересекаются друг с другом. Подобные семейства мы будем называть допустимыми системами кривых.

Говорят, что множество $\Gamma_{j}$ кривых на $S_{0}$ образует гомотопический класс, порожденный кривой $\gamma_{j}$ из допустимой системы $\left\{\gamma_{1}, \ldots, \gamma_{m}\right\}$, если это множество состоит из всех кривых типа I или II, которые свободно гомотопны $\gamma_{j}$ на $S_{0}$. Предположим, что $\alpha:=\left(\alpha_{1}, \ldots, \alpha_{m}\right)$ есть ненулевой вектор с неотрицательными компонентами. Пусть $P$ есть семейство конформно инвариантных метрик $\rho$ на $S_{0}$, удовлетворяющих следующему условию допустимости (см. [47], [105]):

$$
\int_{\gamma} \rho(\zeta)|d \zeta| \geqslant \alpha_{j}
$$

для любых $\gamma \in \Gamma_{j}, j=1, \ldots, m$. (Следуя [105], мы используем в формуле (4) нижний интеграл Дарбу для того, чтобы избежать интегрирования вдоль неспрямляемых кривых, которые могут появиться в результате применения квазиконформного отображения к спрямляемой кривой.)

В случае, когда $P \neq \varnothing$, говорят, что для заданного семейства $\Gamma=\left\{\Gamma_{1}, \ldots\right.$, $\left.\Gamma_{m}\right\}$ на $S_{0}$ и вектора $\alpha$ задача о модуле корректно поставлена, а ее решением 
считается величина, называемая модулем, которая определяется формулой

$$
m\left(S_{0}, \Gamma, \alpha\right)=\inf _{\rho \in P} \iint_{S_{0}} \rho^{2}(\zeta) d \sigma_{\zeta} .
$$

Известно (см. [47]), что экстремальная метрика $\rho^{*}$ в сформулированной задаче о модуле определяется единственным образом. Она отвечает единственному голоморфному квадратичному дифференциалу $\varphi=\varphi(\zeta) d \zeta^{2}$ из пространства $H^{2,0}\left(S_{0}\right)$, имеющему конечные траектории на $S_{0}$, при этом $\rho^{*}(\zeta)=\sqrt{|\varphi(\zeta)|}$. Из определения модуля ясно, что он является конформно инвариантным понятием.

Мы говорим, что гиперболическая двусвязная область $D_{j}$ ассоииирована с гомотопическим классом $\Gamma_{j}$ первого типа $(\mathrm{I})$, если любая простая петля, разделяющая граничные компоненты области $D_{j}$, принадлежит $\Gamma_{j}$. Аналогичное определение можно дать для кривых второго типа. А именно, предположим, что задан четырехугольник $D_{j}$ с различными вершинами 1,4 и 2,3 на двух различных гиперболических компонентах границы поверхности $S_{0}$. Будем говорить, что он ассощиирован с гомотопическим классом $\Gamma_{j}$ второго типа (II), если любая простая дуга, соединяющая граничную компоненту с точками 1,4 с граничной компонентой с точками 2,3 , принадлежит $\Gamma_{j}$.

Критические траектории дифференциала $\varphi=\varphi(\zeta) d \zeta^{2}$ разбивают $S_{0}$ не более чем на $m$ кольцевых областей и четырехугольников, ассоциированных с соответствующими гомотопическими классами кривых. Обозначим их совокупность через $\mathfrak{D}^{*}=\left\{D_{1}^{*}, \ldots, D_{m}^{*}\right\}$ (заметим, что некоторые из четырехугольников, входящих в эту совокупность, могут вырождаться). По аналогии с семействами кривых мы будем называть двусвязные области из этой совокупности областями типа I, а четырехугольники - областями типа II. Любое семейство из $m$ непересекающихся допустимых двусвязных областей и четырехугольников $\mathfrak{D}=\left\{D_{1}, \ldots, D_{m}\right\}$ типов I, II, ассоциированных с гомотопическими классами свободного семейства $Г$, удовлетворяет следующему неравенству:

$$
\sum_{j=1}^{m} \alpha_{j}^{2} M\left(D_{j}\right) \leqslant \sum_{j=1}^{m} \alpha_{j}^{2} M\left(D_{j}^{*}\right),
$$

равенство в котором достигается только для $\mathfrak{D}=\mathfrak{D}^{*}$. Здесь $M\left(D_{j}\right)$ обозначает модуль двусвязной области относительно семейства кривых, разделяющих ее граничные компоненты, или модуль четырехугольника относительно семейства кривых, связывающих противоположные гиперболические компоненты границы $\partial S_{0}$.

Как указано, каждая область $D_{j}^{*}$ совпадает либо с кольцевой областью, либо с четырехугольником в системе траекторий дифференциала $\varphi=\varphi(\zeta) d \zeta^{2}$. Если $D_{j}^{*}$ есть кольцевая область, то в этой области существует конформное отображение $g_{j}$, являющееся решением дифференциального уравнения

$$
\alpha_{j}^{2}\left(\frac{g_{j}^{\prime}(\zeta)}{g_{j}(\zeta)}\right)^{2}=-4 \pi^{2} \varphi(\zeta) .
$$

Оно отображает область $D_{j}^{*}$ на кольцо $\left\{1<|w|<\exp \left(2 \pi M\left(D_{j}^{*}\right)\right)\right\}$. Если $D_{j}^{*}$ является четырехугольником, то в $D_{j}^{*}$ существует конформное отображение $g_{j}$, удовлетворяющее дифференциальному уравнению

$$
\alpha_{j}^{2}\left(g_{j}^{\prime}(\zeta)\right)^{2}=\varphi(\zeta)
$$


Оно отображает область $D_{j}^{*}$ на четырехугольник $\{0<\operatorname{Re} w<1,0<\operatorname{Im} w<$ $\left.M\left(D_{j}^{*}\right)\right\}$. Мы называем систему областей $\mathfrak{D}^{*}$ характеристической системой областей для дифференциала $\varphi=\varphi(\zeta) d \zeta^{2}$.

Таким образом, проблема модуля для некоторого свободного семейства гомотопических классов кривых эквивалентна проблеме построения экстремального разбиения римановой поверхности $S_{0}$ на области указанных типов, ассоциированные с заданным семейством.

Построим на римановой поверхности $S_{0}$ типа $(g, n, l)$ допустимую систему $\left\{\gamma_{1}, \ldots, \gamma_{m}\right\}$ кривых типов I, II, порождающую отвечающее ей свободное семейство $\Gamma=\left\{\Gamma_{1}, \ldots, \Gamma_{m}\right\}$ гомотопических классов кривых. Для заданного свободного семейства Г и фиксированного весового вектора $\alpha$ рассмотрим задачу о модуле (4), (5) и обозначим искомый модуль через $m_{0}=m\left(S_{0}, \Gamma, \alpha\right)$. Пусть оснащенная риманова поверхность $(S, f)$ отвечает точке $x$ пространства Тейхмюллера $T\left(S_{0}\right)$. Ей отвечает квазиконформное отображение $w=f(\zeta): S_{0} \rightarrow S$ с комплексной дилатацией $\mu_{f}=f_{\bar{\zeta}} / f_{\zeta}$. Рассмотрим теперь задачу о модуле (4), (5) для допустимой системы кривых $\left\{f\left(\gamma_{1}\right), \ldots, f\left(\gamma_{m}\right)\right\}$ с тем же весовым вектором $\alpha$ на римановой поверхности $S$. Ей отвечает свободное семейство кривых $\Gamma_{f}$ на поверхности $S$ с модулем $m_{f}=m\left(S, \Gamma_{f}, \alpha\right)$. Если $\varphi_{\mu}(w) d w^{2}(w-$ локальный параметр на $S$ ) есть экстремальный квадратичный дифференциал для задачи о модуле на $S$, то

$$
m_{f}=\iint_{S}\left|\varphi_{\mu}\right| d \sigma_{w}
$$

Тем самым, мы сопоставили оснащенной римановой поверхности $(S, f)$ некоторый функционал $m_{f}$, который мы также будем называть модулем. Так как гомотопический тип кривых на поверхности $S$ однозначно определяется гомотопическим типом отображений, принадлежащих одному и тому же классу эквивалентности $x \in T\left(S_{0}\right)$, то из конформной инвариантности модуля следует, что значение $m_{f}$ зависит только от точки $x \in T\left(S_{0}\right)$, но не от выбора конкретного отображения $f$. Поэтому впредь модуль $m_{f}$ обозначается через $m(x)$, где $x$ пробегает все пространство $T\left(S_{0}\right)$ при фиксированной допустимой системе $\Gamma$, весовом векторе $\alpha$ и начальным условии $m(0)=m_{0}$. Если $\Phi\left(\mu_{1}\right)=\Phi\left(\mu_{2}\right)=x$, то дифференциал $\varphi_{\mu_{1}}$ может быть получен из дифференциала $\varphi_{\mu_{2}}$ с помощью конформного преобразования. Так как квадратичные дифференциалы инвариантны относительно конформных замен параметров, то дифференциалы $\varphi_{\mu_{1}}$ и $\varphi_{\mu_{2}}$ представляют один и тот же дифференциал $\varphi_{x}$ с $\varphi_{0} \equiv \varphi$.

Нас интересует зависимость модуля $m(x)$ от точки $x$ пространства Тейхмюллера $T\left(S_{0}\right)$. Введем обозначение:

$$
t=\inf \|\mu\|_{\infty},
$$

где нижняя грань берется по всем $\mu \in B\left(G_{0}\right)$ таким, что $\Phi(\mu)=x \in T\left(G_{0}\right)$. Обозначим через $\mu^{*} \in B\left(G_{0}\right)$ экстремальный дифференциал Бельтрами, на котором реализуется нижняя грань в $(6):\left\|\mu^{*}\right\|_{\infty}=t$.

ПреДлОЖЕНИЕ 3 [117]. Справедлива следующая формула:

$$
m(x)=m(0)-2 \operatorname{Re} \iint_{\mathbb{D} / G_{0}} \mu^{*}(z) q(z) d \sigma_{z}+o(t),
$$


где $q=q(z) d z^{2}$ есть поднятие экстремального квадратичного дифференииала ч на универсальное накрытие $\mathbb{D}$.

Исследуем локальные гармонические свойства модуля $m(x)$ на пространстве Тейхмюллера $T\left(S_{0}\right)$ исходной римановой поверхности $S_{0}$. Мы предполагаем, что это поверхность рода $g$ с $n$ возможными проколами, удовлетворяющая условию $3 g+n-3>0$.

ПрЕДЛОЖЕНИЕ 4 [118]. Пусть $m=3 g-3+n$. Зафиксируем допустимую систему Г из $m$ кривых и весового вектора $\alpha=\left(\alpha_{1}, \ldots, \alpha_{m}\right)$ с положительными координатами $\alpha_{j}$, имеюшую модуль $m\left(S_{0}, \Gamma, \alpha\right)$. Предположим, что траектории экстремального квадратичного дифференииала $\varphi_{0}$ разбивают поверхность $S_{0}$ на $m$ невырожденных кольщевых областей. Тогда существует окрестность $\Omega$ исходной точки пространства Тейхмюллера $T\left(S_{0}\right)$ такая, что ограничение модуля $m(x)$ на эту окрестность является гармонической функияей.

Простые контрпримеры показывают, что функция $m(x)$ не является плюригармонической.

Однако справедливо следующее предложение.

ПрЕДЛОЖЕНИЕ 5 [118]. Продолжсение $m(x)$ модуля $m_{0}=m\left(S_{0}, \Gamma, \alpha\right)$ является субгармонической функиией во всем пространстве Тейхмюллера $T\left(S_{0}\right)$ для произвольной допустимой системы Г и любого весового вектора $\alpha$.

Метрика Тейхмюллера допускает переопределение в терминах модулей. Пусть $m\left(S_{0}, \Gamma, \alpha\right)$ есть модуль заданной допустимой системы Г кривых типов I, II на $S_{0}$ с весовым вектором $\alpha$, и пусть $m(x)$ обозначает продолжение этого модуля на пространство Тейхмюллера $T\left(S_{0}\right)$. Справедливо следующее пердложение.

ПреДЛОЖеНИЕ 6 [115]. Метрика Тейхмюллера $\tau_{T}(x, y)$ на пространстве Тейхмюллера $T\left(S_{0}\right)$ описывается следующим образом:

$$
\tau_{T}(x, y)=\sup _{\Gamma, \alpha} \frac{1}{2}\left|\log \frac{m(x)}{m(y)}\right|=\sup _{\Gamma, \alpha} \frac{1}{2}\left|\log \frac{m\left(f_{1}\left(S_{0}\right), f_{1}(\Gamma), \alpha\right)}{m\left(f_{2}\left(S_{0}\right), f_{2}(\Gamma), \alpha\right)}\right|,
$$

где $\left(f_{1}\left(S_{0}\right), f_{1}\right)$ и $\left(f_{2}\left(S_{0}\right), f_{2}\right)$ - оснащенные римановы поверхности, отвечающие точкам $x$ и у соответственно.

Похожее описание было получено Керкхофом [52] для компактных и проколотых римановых поверхностей в терминах экстремальной длины Альфорса-Бёрлинга.

Следующая теорема, основанная на гармонических свойствах функционала $m(x)$, дает достаточное условие геодезичности дисков Тейхмюллера или их поддисков в метрике Каратеодори (см. п. 2.5).

Tеорема 1 [116]. Пусть $X_{r}=\left\{x: x \in T(g, n), \tau_{T}(0, x)<r\right\}$. Пусть $\varphi$ является экстремальным квадратичным дифференциалом для функиионала $m(x)$ в точке $x=0, a q$-его поднятие на универсальное накрытие $U$. Если функиия $m(x)$ плюригармонична на $X_{r}$, то на диске $\Delta_{q} \cap X_{r}$ метрика ТейхмюллераКобаяси $\tau_{T}$ и метрика Каратеодори $c_{X_{r}}$ совпадают. В частности, это утверждение верно для всего пространства $T(g, n)$ при $r \rightarrow \infty u X_{r} \rightarrow T(g, n)$. 
Задача о модуле (4), (5) тесно связана с принципом Дирихле. А именно, допустим, что нам заданы риманова поверхность $S_{0}$ конечного типа $(g, n, l)$, наделенная комплексной структурой, и свободное семейство гомотопических классов кривых первого и второго типов на $S_{0}$. Задача Дирихле заключается в отыскании минимума $L_{1}$-нормы квадратичного дифференциала $\varphi$, отвечающего комплексной структуре $S_{0}$, среди всех дифференциалов заданного гомотопического типа, принадлежащих данному свободному семейству. Траектории этого дифференциала имеют в метрике $\sqrt{|\varphi|}$ длину, не превосходящую некоторого вполне определенного числа. Принцип Дирихле утверждает, что существует единственный дифференциал, на котором достигается указанный минимум. В такой постановке принцип Дирихле является частным случаем задачи о модуле в ситуации, когда рассматриваются только метрики вида $\sqrt{|\varphi|}$ из класса всех допустимых метрик. Поэтому не удивительно, что решения обеих проблем приводят к одной и той же метрике (см. [48] и [103]).

Подход, близкий к изложенному, был использован Тёрстоном (см. книгу [29], основанную на материале лекций Тёрстона в Орсэ в 1976-1977 гг.). Подход Тёрстона также основан на принципе Дирихле. Более подробно, в пространстве всех гомотопических классов кривых вводятся слоения с мерой и ищется минимум $L^{1}$-нормы в пространстве дифференциалов кривых заданного гомотопического типа, имеющих в метрике $|\operatorname{Im} \sqrt{\varphi}|$ высоту, не превосходящую заданной. Слоение строится путем формального умножения гомотопических классов на положительные числа, так что каждому классу отвечает отдельный луч слоения.

Норма слоения с мерой играет роль, аналогичную роли нормы экстремального квадратичного дифференциала в задаче о модуле. Пользуясь указанной нормой, можно получить описание метрики Тейхмюллера, исследовать гармонические свойства и связь с инвариантными метриками Кобаяси и Каратеодори так же, как в задаче о модуле.

\section{9. Дополнение: пространства Тейхмюллера и теория струн.}

В классической ньютоновой механике точечная частица движется, в отсутствие внешних сил, по кратчайшей траектории (геодезической), соединяющей ее начальное положение с конечным. При переходе к квантовому случаю ситуация кардинальным образом меняется. Согласно концепции, предложенной Фейнманом в 1948 г., элементарные частицы могут двигаться вдоль любых траекторий, соединяющих начальное положение с конечным. Более точно, каждой траектории можно приписать плотность вероятности, которая тем выше, чем "короче" траектория или, в лагранжевой постановке, чем меньше действие. При этом частицы по-прежнему рассматриваются как топологические точки, однако в квантовой механике они могут обладать и дополнительной внутренней структурой.

Cтруна есть 1-мерный объект, движущийся в плоском $D$-мерном пространстве-времени $\mathfrak{M}$, первая координата которого отождествляется со временем. В процессе движения струна заметает 2-мерную поверхность $M$ в пространстве $\mathfrak{M}$, которая называется мировой поверхностъю струны, по аналогии с мировой линией ньютоновой частицы. Попытаемся ввести на пространстве всех 
поверхностей, соединяющих начальное и конечное "положения" струны, плотность вероятности, которая обладает следующим свойством: она тем выше, чем меньше действие.

Если обозначить действие через $S[\phi]$, где $\phi$ есть величина, характеризующая поверхность (ее роль дальше играет метрика), то гауссово распределение будет давать простейший фейнмановский интеграл по траекториям

$$
Z=\int_{\text {ini }}^{\text {fin }} D \phi e^{-(1 / \hbar) S[\phi]},
$$

или статистическую сумму, где ћ обозначает постоянную Планка, а $D \phi$ играет роль меры, являясь, грубо говоря, локальным произведением $\prod_{x \in \mathfrak{M}} d \mu(\phi(x))$ мер на пространстве $\mathfrak{M}$. Символ $\int D \phi$ является краткой записью бесконечномерного интеграла по всем возможным полевым конфигурациям на пространстве-времени и, строго говоря, не определен как корректный математический объект. Причина в том, что на бесконечномерном векторном пространстве не существует трансляционно инвариантной меры. Поэтому указанный интеграл по траекториям следует рассматривать как некий формальный символ, строгий математический смысл которому удается придать только в некоторых специальных случаях. Например, в размерности $D=26$ его можно математически корректно определить как интеграл по пространству Тейхмюллера по мере Вейля-Петерсона.

Параметризуем мировую поверхность струны $M$ параметрами $(\tau, \sigma)$, а через $x^{\mu}, \mu=0,1, \ldots, D-1$, обозначим координаты на пространстве-времени $\mathfrak{M}$. В то время как параметры $(\tau, \sigma)$ не имеют прямого физического смысла, функции $x^{\mu}(\tau, \sigma)$, отмечающие положение точки на мировой поверхности, характеризуют движение струны в пространстве-времени $\mathfrak{M}$ и потому именуются динамическими переменными.

Струна называется замкнутой, если динамические переменные являются периодическими функциями от $\sigma$, и открытой в противоположном случае. Параметр $\tau$ принимает значение на всей прямой $(-\infty, \infty)$ или полупрямой $[0, \infty)$. В дальнейшем мы ограничиваемся замкнутыми струнами.

Предположим, что $\mathfrak{M}$ есть $D$-мерное векторное пространство, наделенное лоренцевой метрикой $d s_{\mathfrak{M}}$ сигнатуры $(1, D-1)$, так что

$$
d s_{\mathfrak{M}}^{2}=\eta_{\mu \nu} d x^{\mu} d x^{\nu}, \quad \eta_{\mu \nu}=\left(\begin{array}{cccc}
-1 & 0 & \ldots & 0 \\
0 & 1 & \ldots & 0 \\
\ldots & \ldots & \ldots & \ldots \\
0 & 0 & \ldots & 1
\end{array}\right)
$$

Рассмотрим следующую упрощенную модель замкнутой бозонной струны. Введем динамические переменные $x^{\mu}\left(\sigma^{\alpha}\right)$, где $\mu=0,1, \ldots, D-1, \alpha=0,1$. Координаты $\sigma^{0}=\tau, \sigma^{1}=\sigma$ определены на каноническом цилиндре $C=\{\tau \in(-\infty, \infty)$, $\sigma \in[0,2 \pi)\}$, наделенном внутренней метрикой

$$
d s_{C}^{2}=\eta_{\alpha \beta} d \sigma^{\alpha} d \sigma^{\beta}, \quad \eta_{\alpha \beta}=\left(\begin{array}{cc}
-1 & 0 \\
0 & 1
\end{array}\right) .
$$


Предполагается, что динамические переменные задают $C^{\infty}$-гладкое вложение цилиндра $C$ в пространство $\mathfrak{M}$, так что мировую поверхность $M$ можно рассматривать как гладкое многообразие, вложенное в $\mathfrak{M}$. Индуцированная метрика $d s_{\mathrm{ws}}$ на мировой поверхности имеет вид

$$
d s_{\mathrm{ws}}^{2}=\eta_{\mu \nu} \frac{\partial x^{\mu}}{\partial \sigma^{\alpha}} \frac{\partial x^{\nu}}{\partial \sigma^{\beta}} d \sigma^{\alpha} d \sigma^{\beta}=g_{\alpha \beta} d \sigma^{\alpha} d \sigma^{\beta} .
$$

Динамические переменные $x^{\mu}(\tau, \sigma)$ удовлетворяют уравнениям движения, вытекающим из приниипа наименьшего действия.

В лагранжевой механике физическая система характеризуется конфигурационным пространством $\mathfrak{N}$ и лагранжсаном $L$, являющимся гладкой вещественнозначной функцией на прямом произведении $T \mathfrak{N} \times \mathbb{R}$ касательного расслоения $T \mathfrak{N}$ и вещественной оси $\mathbb{R}$. Обозначим через $P \mathfrak{N}$ пространство траекторий, т. е. пространство всех гладких путей с фиксированными начальной ("ini") и конечной ("end") точками. Это пространство имеет структуру бесконечномерного многообразия Фреше. Функиионал действия $S$ является вещественнозначным интегральным функционалом, задаваемым равенством $S=$ $\int_{\text {ini }}^{\text {fin }} L d t$, где $t$ - вещественный параметр на кривой. В классической механике действие равно разности между кинетической и потенциальной энергиями. В релятивистской механике прямого аналога энергии ввести не удается, поэтому Намбу в 1970 г. [75] и Гото в 1971 г. [36] предложили взять в качестве простейшего действия площадь мировой поверхности $M$. Это действие, известное под названием действия Намбу-Гото, задается формулой

$$
S_{\mathrm{NG}}=-T \int_{M} d x=-T \int_{C} d \sigma^{2} \sqrt{\left|\operatorname{det} g_{\alpha \beta}\right|},
$$

где $C$ - канонический цилиндр, а величина $T$, называемая натяжением струны, имеет размерность массы на единицу длины. (Заметим, что знак "минус" перед интегралом происходит из лоренцевой метрики.)

Геодезическое движение струны определяется динамическими переменными, удовлетворяющими следующим уравнениям Эйлера-Лагранжа:

$$
\frac{1}{\sqrt{\left|\operatorname{det} g_{\alpha \beta}\right|}} \frac{\partial}{\partial \sigma^{\alpha}}\left(\sqrt{\left|\operatorname{det} g_{\alpha \beta}\right|} g^{\alpha \beta} \frac{\partial x^{\mu}}{\partial \sigma^{\beta}}\right)=0, \quad \mu=0, \ldots, D-1,
$$

где поднятие и опускание индексов у метрик $g^{\alpha \beta}$ и $g_{\alpha \beta}$ осуществляются с помощью внутренней лоренцевой метрики. Метрика $g_{\alpha \beta}$ определена на мировой поверхности $M$, вложенной в $\mathfrak{M}$, и зависит от переменных $x^{\mu}$.

Заметим, что уравнения Эйлера-Лагранжа (8) для динамических переменных $x^{\mu}$ сильно нелинейны, а наличие квадратного корня под интегралом создает дополнительные трудности при квантовании. Для преодоления этих трудностей Поляков в работе [88] 1981 г. предложил использовать метод, сходный с методом множителей Лагранжа. Он ввел на мировой поверхности в качестве дополнительного переменного метрику $d s^{2}=h_{\alpha \beta}(\tau, \sigma) d \sigma^{\alpha} d \sigma^{\beta}$, так что действие приняло следующий вид:

$$
S_{\mathrm{P}}=-\frac{T}{2} \int_{C} d \sigma^{2} \sqrt{\left|\operatorname{det} h_{\alpha \beta}\right|} h^{\alpha \beta} \frac{\partial x^{\mu}}{\partial \sigma^{\alpha}} \frac{\partial x^{\nu}}{\partial \sigma^{\beta}} .
$$


Уравнения Эйлера-Лагранжа (относительно вариации динамических переменных) для действия Полякова $S_{\mathrm{P}}$ формально имеют тот же вид, что и для действия Намбу-Гото $S_{\mathrm{NG}}$ :

$$
\frac{1}{\sqrt{\left|\operatorname{det} h_{\alpha \beta}\right|}} \frac{\partial}{\partial \sigma^{\alpha}}\left(\sqrt{\left|\operatorname{det} h_{\alpha \beta}\right|} h^{\alpha \beta} \frac{\partial x^{\mu}}{\partial \sigma^{\beta}}\right)=0,
$$

однако смысл их уже другой, поскольку метрика $h=\left(h_{\alpha \beta}(\tau, \sigma)\right)$ зависит только от $(\tau, \sigma)$, но не зависит от $x^{\mu}$. Поэтому уравнения $(9)$ линейны по $x^{\mu}(\tau, \sigma)$.

Однако за введение дополнительной метрики $h=\left(h_{\alpha \beta}\right)$ приходится платить тем, что в нашей системе появляется три дополнительных степени свободы (три - ввиду симметричности метрики $h$ ). От них можно избавиться с помощью следующих дополнительных уравнений движения на $h$ :

$$
\frac{\delta S_{\mathrm{P}}}{\delta h^{\alpha \beta}}=0
$$

где в левой части стоит функциональная производная. Из уравнений (9), (10) выводятся необходимые условия для существования минимума действия. А именно, определим двумерный тензор энергии-импульса как

$$
T_{\alpha \beta}=\frac{-2}{T} \frac{1}{\sqrt{\left|\operatorname{det} h_{\alpha \beta}\right|}} \frac{\delta S_{\mathrm{P}}}{\delta h^{\alpha \beta}} .
$$

Тогда из уравнений движения следует, что $T_{\alpha \beta}=0$. Заметим, что при этом условии $S_{\mathrm{P}}=S_{\mathrm{NG}}$ (тогда как в общем случае $\left.S_{\mathrm{P}} \geqslant S_{\mathrm{NG}}\right)$.

Согласно одному из руководящих принципов физики, известному под названием "теоремы Нётер", симметрии пространства-времени должны порождать законы сохранения. (Заметим в скобках, что указанную "теорему" следует рассматривать скорее как эвристический принцип, нежели как теорему, поскольку собственно теорема Нётер, доказанная в 1918 г. [78], относится к очень частному случаю сформулированного принципа.)

$\mathrm{K}$ симметриям действия Полякова $S_{\mathrm{P}}$, т. е. преобразованиям, оставляющим это действие инвариантным, относятся:

- глобальные симметрии:

- преобразования Пуанкаре

$$
x^{\mu} \rightarrow x^{\mu}+b^{\mu}, \quad x^{\mu} \rightarrow x^{\mu}+\omega_{\nu}^{\mu} x^{\nu},
$$

где $\omega_{\nu}^{\mu}=-\omega_{\mu}^{\nu}-$ инфинитезимальные преобразования Лоренца;

- локальные симметрии:

- преобразования репараметризации, задаваемые диффеоморфизмами в $2 \mathrm{D}$ измерениях $\sigma^{\alpha} \rightarrow \tilde{\sigma}^{\alpha}(\tau, \sigma)$, сохраняющие площадь

$$
d \tilde{\sigma}^{2} \sqrt{|\operatorname{det} \tilde{h}|}=d \sigma^{2} \sqrt{|\operatorname{det} h|} ;
$$

- вейлевское масштабирование

$$
h_{\alpha \beta} d \sigma^{\alpha} d \sigma^{\beta} \rightarrow e^{\rho(\tau, \sigma)} h_{\alpha \beta} d \sigma^{\alpha} d \sigma^{\beta},
$$

оставляющее произведение $\sqrt{|\operatorname{det} h|} h_{\alpha \beta}$ инвариантным. 
Инвариантность $S_{\mathrm{P}}$ относительно преобразований Пуанкаре и репараметризации позволяет нам выбрать калибровку, в которой три независимых компоненты метрики $h$ выражаются через одну функцию, обычно отождествляемую с конформно плоской калибровкой: $h_{\alpha \beta}=e^{\rho(\tau, \sigma)} \eta_{\alpha \beta}$. Подставляя это выражение в действие Полякова, получим

$$
S_{\mathrm{P}}=-\frac{T}{2} \int_{C} d \sigma^{2} \eta^{\alpha \beta} \eta_{\mu \nu} \frac{\partial x^{\mu}}{\partial \sigma^{\alpha}} \frac{\partial x^{\nu}}{\partial \sigma^{\beta}},
$$

поэтому при фиксированной калибровке действие является функционалом, квадратичным по $x$. Варьируя его по $x$, получим уравнение движения для свободного поля

$$
\ddot{x}^{\mu}-\left(x^{\mu}\right)^{\prime \prime}=0,
$$

где $\dot{x}$ обозначает производную по $\tau$, а $x^{\prime}-$ производную по $\sigma$.

Из вейлевской инвариантности следует, что $S_{\mathrm{P}}$ не зависит от $\rho(\tau, \sigma)$. Поэтому вариация $\delta S_{\mathrm{P}} / \delta \rho=h^{\alpha \beta} T_{\alpha \beta}=T_{\alpha}^{\alpha}$ обращается в нуль, откуда следует, что тензор $T_{\alpha \beta}$ является бесследовым.

Пользуясь тем, что $T_{\alpha \alpha}=0$, можно вывести отсюда соотношения $T_{01}=$ $T_{10}=\dot{x}_{\mu}\left(x_{\mu}\right)^{\prime}=0$ и

$$
T_{00}=T_{11}=\frac{1}{2}\left(\dot{x}_{\mu} \dot{x}^{\mu}+x_{\mu}^{\prime}\left(x^{\mu}\right)^{\prime}\right)=0 .
$$

Это приводит к уравнениям $\left(\dot{x} \pm x^{\prime}\right)^{2}=0$, называемым связями Вирасоро. Уравнение (11) со связями (12) (плюс граничные условия) полностью описывает движение бозонной струны.

Воспользуемся периодическими граничными условиями $x^{\mu}(\tau, \sigma)=x^{\mu}(\tau, \sigma+$ $2 \pi)$. Тогда общее решение волнового уравнения движения (11) будет иметь вид

$$
x^{\mu}(\tau, \sigma)=x_{\mathrm{R}}^{\mu}(\tau-\sigma)+x_{\mathrm{L}}^{\mu}(\tau+\sigma) .
$$

Покажем, что центр масс мировой поверхности движется как свободная частица. Пользуясь граничными условиями и отделяя линейную часть, представим решение в виде рядов Фурье следующего вида:

$$
\begin{aligned}
& x_{\mathrm{R}}^{\mu}(\tau-\sigma)=\frac{1}{2} x_{0}^{\mu}+\frac{1}{2 \pi T}(\tau-\sigma) p^{\mu}+\frac{i}{\sqrt{2 \pi T}} \sum_{n \neq 0} \frac{1}{n} \alpha_{n}^{\mu} e^{-i n(\tau-\sigma)}, \\
& x_{\mathrm{L}}^{\mu}(\tau+\sigma)=\frac{1}{2} x_{0}^{\mu}+\frac{1}{2 \pi T}(\tau+\sigma) p^{\mu}+\frac{i}{\sqrt{2 \pi T}} \sum_{n \neq 0} \frac{1}{n} \beta_{n}^{\mu} e^{-i n(\tau+\sigma)},
\end{aligned}
$$

где $x_{0}^{\mu}$ - центр масс, а $p^{\mu}$-импульс. Функции $x_{\mathrm{R}}^{\mu}$ и $x_{\mathrm{L}}^{\mu}$ являются вещественными, поэтому $\bar{\alpha}_{n}^{\mu}=\alpha_{-n}^{\mu}$ и $\bar{\beta}_{n}^{\mu}=\beta_{-n}^{\mu}$. Указанные коэффициенты физики называют осиилляторами.

Позиция центра масс струны определяется формулой

$$
X^{\mu}=\frac{1}{2 \pi} \int_{0}^{2 \pi} x^{\mu}(\tau, \sigma) d \sigma=x_{0}^{\mu}+\frac{p^{\mu}}{\pi T} \tau,
$$

которая показывает, что центр масс движется как свободная частица с начальным положением $x_{0}^{\mu}$. Импульс центра масс записывается в виде

$$
P^{\mu}=\int_{0}^{2 \pi} \Pi^{\mu} d \sigma=p^{\mu}
$$

где $\Pi^{\mu}:=(T / 2) \dot{x}^{\mu}$. 
В классической гамильтоновой механике действие $S$ задается интегралом по времени от функции Лагранжа $S=\int_{t_{0}}^{t_{1}} L(x, \dot{x}) d t$. В нашем случае эта функция имеет вид

$$
L=-\frac{T}{2} \int_{0}^{2 \pi} \eta^{\alpha \beta} \eta_{\mu \nu} \frac{\partial x^{\mu}}{\partial \sigma^{\alpha}} \frac{\partial x^{\nu}}{\partial \sigma^{\beta}} d \sigma .
$$

Функция Гамильтона равна

$$
H=\int_{0}^{2 \pi}\left(\dot{x}^{\mu} \Pi_{\mu}\right) d \sigma-L=\frac{T}{2} \int_{0}^{2 \pi}\left(\dot{x}^{\mu} \dot{x}_{\mu}+\left(x^{\mu}\right)^{\prime} x_{\mu}^{\prime}\right) d \sigma .
$$

Пользуясь следующим тождеством для кронекеровской дельта-функции:

$$
\frac{1}{2 \pi} \int_{0}^{2 \pi} e^{i(n-m) \sigma} d \sigma=\delta_{n, m}
$$

получаем выражение для функции Гамильтона в терминах осцилляторов

$$
H=\frac{1}{2} \sum_{n \in \mathbb{Z}}\left(\alpha_{-n} \alpha_{n}+\beta_{-n} \beta_{n}\right),
$$

где мы положили $\alpha_{0}^{\mu}=\beta_{0}^{\mu}=p^{\mu} /(2 \pi T)$.

Стандартная скобка Гейзенберга-Пуассона-Дирака в фазовых координатах задается выражением

$$
\begin{aligned}
& \left\{x^{\mu}(\tau, \sigma), \dot{x}^{\nu}\left(\tau, \sigma^{\prime}\right)\right\}=\frac{1}{T} \eta^{\mu, \nu} \delta\left(\sigma-\sigma^{\prime}\right), \\
& \left\{x^{\mu}(\tau, \sigma), x^{\nu}\left(\tau, \sigma^{\prime}\right)\right\}=\left\{\dot{x}^{\mu}(\tau, \sigma), \dot{x}^{\nu}\left(\tau, \sigma^{\prime}\right)\right\}=0 .
\end{aligned}
$$

Удобно перейти здесь к световым координатам на $C$, полагая скорость света равной 1. Эти координаты задаются равенством $\zeta_{ \pm}=\sigma \pm \tau$, так что плоская метрика $d s_{C}^{2}$ в этих координатах записывается в виде $d s_{C}^{2}=d \zeta_{+} d \zeta_{-}$. Компоненты метрики $\eta_{\alpha \beta}$ в световых координатах приобретают вид $\eta_{++}=\eta_{--}=0$ и $\eta_{+-}=\eta_{-+}=1 / 2$, тогда как операторы дифференцирования задаются формулой $\partial_{ \pm}=\left(\partial_{\sigma} \mp \partial_{\tau}\right) / 2$.

Образующие Вирасоро определяются равенствами

$$
\begin{aligned}
& L_{m}=\frac{1}{2} \int_{0}^{2 \pi} T_{++} e^{i m(\tau-\sigma)} d \sigma=\frac{1}{2} \sum_{n \in \mathbb{Z}} \alpha_{m-n} \alpha_{n}, \\
& \widetilde{L}_{m}=\frac{1}{2} \int_{0}^{2 \pi} T_{--} e^{i m(\tau+\sigma)} d \sigma=\frac{1}{2} \sum_{n \in \mathbb{Z}} \beta_{m-n} \beta_{n},
\end{aligned}
$$

где $T_{++}$и $T_{--}$- главные диагональные компоненты тензора энергии-импульса в вещественных световых координатах и $\alpha_{0}^{\mu}=\beta_{0}^{\mu}=p^{\mu} /(2 \pi T)$. Коммутационные соотношения для операторов $L_{n}$ относительно скобки Пуассона имеют вид

$$
\left\{L_{m}, L_{n}\right\}=i(n-m) L_{n+m}, \quad\left\{\widetilde{L}_{m}, \widetilde{L}_{n}\right\}=i(n-m) \widetilde{L}_{n+m}, \quad\left\{L_{m}, \widetilde{L}_{n}\right\}=0 .
$$

Следующей полезной операцией является так называемый виковский пово$p o m$, при котором нефизическое время $\tau$ заменяется чисто мнимым $i \tau$. При этом световые координаты $\zeta_{+}, \zeta_{-}$заменяются координатами $z, \bar{z}$ соответствен- 
но, где $z=\sigma+i \tau$, а операторы дифференцирования - операторами $\partial_{+} \rightarrow \partial_{z}$, $\partial_{-} \rightarrow \partial_{\bar{z}}$. Тогда действие Полякова в конформной калибровке превращается в

$$
S_{\mathrm{P}}=-2 T \int_{C} \frac{d z \wedge d \bar{z}}{2 i} \eta_{\mu \nu} \partial_{z} x^{\mu} \partial_{\bar{z}} x^{\nu},
$$

а уравнение движения переходит в уравнение Лапласа $\partial_{z \bar{z}}^{2} x^{\mu}=0$. Компоненты тензора энергии-импульса записываются в виде

$$
\begin{aligned}
& T_{z z}=\frac{1}{2} \eta_{\mu \nu}\left(\left(x^{\mu}\right)^{\prime}-i \dot{x}^{\mu}\right)\left(\left(x^{\nu}\right)^{\prime}-i \dot{x}^{\nu}\right)=T_{00}+2 i T_{10}, \\
& T_{\bar{z} \bar{z}}=\frac{1}{2} \eta_{\mu \nu}\left(\left(x^{\mu}\right)^{\prime}+i \dot{x}^{\mu}\right)\left(\left(x^{\nu}\right)^{\prime}+i \dot{x}^{\nu}\right)=T_{00}-2 i T_{10},
\end{aligned}
$$

и $T_{z \bar{z}}=T_{\bar{z} z}=0$. Тем самым, связи Вирасоро в этих координатах сводятся к уравнениям $T_{z z}=T_{\bar{z} \bar{z}}=0$.

Из инвариантности $S_{\mathrm{P}}$ относительно инфинитезимальной репараметризации: $\sigma^{\alpha} \rightarrow \tilde{\sigma}^{\alpha}+\varepsilon^{\alpha}(\sigma)$ вытекает закон сохранения для тензора энергии-импульса $\nabla^{\alpha} T_{\alpha \beta}=0$, который в $z$-координатах превращается в

$$
\partial_{\bar{z}} T_{z z}+\partial_{z} T_{\bar{z} z}=\partial_{z} T_{\bar{z} \bar{z}}+\partial_{\bar{z}} T_{z \bar{z}}=0
$$

откуда, пользуясь соотношением $T_{z \bar{z}}=T_{\bar{z} z}=0$, получаем, что $T_{z z}$ является аналитической, а $T_{\bar{z} \bar{z}}-$ антианалитической компонентой $T$.

Поэтому в комплексных координатах

$$
\begin{aligned}
& \partial_{z} x^{\mu}=\frac{1}{\sqrt{4 \pi T}} \sum_{n \in \mathbb{Z}} \beta_{n}^{\mu} e^{-i n z}, \\
& \partial_{\bar{z}} x^{\mu}=\frac{1}{\sqrt{4 \pi T}} \sum_{n \in \mathbb{Z}} \alpha_{n}^{\mu} e^{-i n \bar{z}} .
\end{aligned}
$$

Тем самым, виковский поворот приводит к комплексным координатам, в которых операторы Вирасоро $L_{n}$ становятся коэффициентами формальных лорановских разложений

$$
T_{z z}=\sum_{n \in \mathbb{Z}} \frac{L_{n}}{z^{n+2}},
$$

а коммутационные соотношения для этих операторов приобретают вид

$$
\left\{L_{m}, L_{n}\right\}=i(n-m) L_{n+m} .
$$

Иначе говоря, операторы $\left\{L_{n}\right\}$ являются образующими алгебрь Buтma.

Вернемся к эвристической идее интеграла (7). Предположим, что у нас имеются некоторые "входящая" $\ell_{\text {inc }}$ и "выходящая" $\ell_{\text {out }}$ струны. Для того чтобы построить мировую поверхность, мы должны заменить $C$ на риманову поверхность $\Sigma$ гиперболического типа с фиксированной ориентированной границей $\ell=\ell_{\text {inc }} \cup \ell_{\text {out }}$. Затем заменить плоское пространство (евклидово или Минковского) в образе на искривленное, т. е. ввести соответственно риманову или лоренцеву метрику $h(x)=h_{\mu \nu} d z^{\mu} d \bar{z}^{\nu}$, где $z^{\mu}$ - локальные координаты на $\Sigma$, a $x: \Sigma \rightarrow \mathfrak{M}$. Статсумма $Z[\ell]$ замкнутой бозонной струны Полякова задается рядом теории возмущений $Z[\ell]=\sum_{g \geqslant 0} Z_{g}[\ell]$ по роду $g$ поверхности $\Sigma$, где

$$
\begin{aligned}
Z_{g}[\ell] & =\int D x D h e^{-S_{\mathrm{P}}(x, h)}, \\
S_{\mathrm{P}} & =-2 T \int_{\Sigma} \frac{d z \wedge d \bar{z}}{2 i} \sqrt{|h|} h^{\mu \nu} \partial_{z} x^{\mu} \partial_{\bar{z}} x^{\nu},
\end{aligned}
$$


так что интегрирование в $S_{\mathrm{P}}$ ведется по поверхности $\Sigma$ и можно при этом пользоваться конформными координатами. Интеграл по траекториям в $Z_{g}[\ell]$ берется по пространству римановых поверхностей $\Sigma$ в $\mathfrak{M}$. Для того чтобы строго его определить, заменим метрику $h$ метрикой $\hat{h}$ постоянной кривизны на $\Sigma$. Пространство таких метрик изоморфно пространству комплексных структур на $\Sigma$, параметризованному пространством модулей $M(g, m)$, где $m$ отвечает числу петель в $\ell$. Тогда интеграл по траекториям в статсумме $Z_{g}[\ell]$, взятый по всем метрикам, сведется к конечномерному интегралу по пространству комплексных структур $M(g, m)$.

Обозначим через $K$ каноническое линейное расслоение на поверхности $\Sigma$ и определим оператор $\bar{\partial}_{-1}: T_{\mathbb{C}}^{(1,0)} \Sigma \rightarrow T_{\mathbb{C}}^{(1,0)} \Sigma \otimes \bar{K}$. С учетом изоморфизма $K^{-1} \rightarrow \bar{K}$, положим:

$$
\bar{\partial}_{-1}: \varphi(z) \frac{\partial}{\partial z} \mapsto \frac{1}{\rho^{2}(z)} \varphi_{\bar{z}}(z)
$$

Здесь $T_{\mathbb{C}}^{(1,0)} \Sigma$ есть голоморфное касательное расслоение, а $\rho^{2} d z \otimes d \bar{z}-$ конформная метрика на $\Sigma$, т. е. не обращающееся в нуль сечение расслоения $K \otimes \bar{K}$. Оператор, сопряженный к $\bar{\partial}_{-1}$ относительно эрмитова произведения Вейля-Петерсона, совпадает с $\bar{\partial}_{-1}^{*}$.

В этих обозначениях получаем следующее формальное выражение для статсуммы (см., например, [42], [49]):

$$
Z_{g}[\ell]=\int_{C F} d \sigma \int_{T(g, m)} d_{\mathrm{WP}} J(\hat{h})\left(\frac{\operatorname{det} \Delta_{\hat{h}}}{\int_{\Sigma} \sqrt{\hat{h}}}\right)^{-D / 2}\left(\operatorname{det} \bar{\partial}_{-1}^{*} \bar{\partial}_{-1}\right),
$$

где $d \sigma$ есть формальная мера на пространстве конформных факторов $(C F)$, возникающая из-за конформной аномалии, a $d_{\mathrm{WP}}$ - мера Вейля-Петерсона:

$$
d_{\mathrm{WP}}=\frac{d t_{1} \wedge \cdots \wedge d t_{6 g-6+3 m}}{\operatorname{det}\left(q_{i}, q_{j}\right)} .
$$

Мера Вейля-Петерсона инвариантна относительно действия модулярной группы, поэтому интегрирование ведется по пространству модулей Римана $M_{g, m}$. Квадратичные дифференциалы $q_{j}$ образуют базис в пространстве $T^{*} T(g, m)$, и $t_{j}$ - координаты в этом базисе. Величина $J(\hat{h})$ называется детерминантом Фаддеева-Попова, его можно переписать в виде функционального интеграла по полям, являющимся духами Фаддеева-Попова [28], [42], [49]. Главный результат бозонной теории струн утверждает, что в критической размерности $D=26$ интеграл не зависит от конформного множителя, поэтому можно убрать интегрирование по пространству конформных множителей, благодаря чему статсумма становится корректно определенным интегралом.

Квантование пространства Тейхмюллера $T(S)=T(g, n)$, отвечающего компактной римановой поверхности $S$ рода $g$ с $n$ проколами, было осуществлено независимо Чеховым и Фоком в [31] и Кашаевым в [51]. Чехов и Фок использовали специальные координаты (так называемые "shear coordinates") на касательном расслоении к пространству Тейхмюллера, тогда как Кашаев работал на декорированных пространствах Тейхмюллера и пользовался введенными Пеннером координатами (так называемой $\lambda$-длиной). Го и Лю [39] связали вместе два подхода, сначала обобщив алгебру Кашаева, являющуюся 
некоммутативной деформацией алгебры рациональных функций в координатах Кашаева, а затем установив соответствие между координатами Чехова-Фока и координатами Кашаева. Более подробно об этих вопросах см. [39] и [84; т. 1, часть D].

\section{3. Универсальное пространство Тейхмюллера}

Понятие универсального пространства Тейхмюллера было введено Берсом [17] в 1968 г. Своим названием оно обязано тому, что все классические пространства Тейхмюллера, рассмотренные в двух предыдущих разделах работы, вкладываются в универсальное пространство в виде комплексных подмногообразий.

Прежде чем дать определение универсального пространства Тейхмюллера, напомним необходимые для этого сведения о квазисимметричных гомеморфизмах. Эти сведения, так же как и другие результаты по теории квазиконформных отображений, можно найти в замечательных лекциях Альфорса [9].

3.1. Квазисимметричные гомеоморфизмы. Как известно, произвольный квазиконформный гомеоморфизм жордановой области $D \subset \widehat{\mathbb{C}}$ на жорданову область $D^{\prime} \subset \widehat{\mathbb{C}}$ непрерывно продолжается на границу $\partial D$ до гомеоморфизма замыканий $\bar{D} \rightarrow \overline{D^{\prime}}$. Спрашивается, когда верно обратное утверждение, т. е. когда заданный гомеоморфизм границ областей допускает продолжение до квазиконформного гомеоморфизма самих областей?

Разберем этот вопрос сначала в случае, когда обе области совпадают с верхней полуплоскостью $H=\{z \in \mathbb{C}: \operatorname{Im} z>0\}$. Граничное значение квазиконформного гомеоморфизма $H \rightarrow H$ является гомеоморфизмом $\mathbb{R} \rightarrow \mathbb{R}$, сохраняющим ориентацию. Монотонно возрастающий гомеоморфизм $f: \mathbb{R} \rightarrow \mathbb{R}$ назовем $k$-квазисимметричным, если

$$
\frac{1}{k} \leqslant \frac{f(x+t)-f(x)}{f(x)-f(x-t)} \leqslant k, \quad x \in \mathbb{R},
$$

для всех $t>0$.

Из определения квазиконформности немедленно следует, что граничное значение $K$-квазиконформного гомеоморфизма $H \rightarrow H$ является $k$-квазисимметричным гомеоморфизмом $\mathbb{R} \rightarrow \mathbb{R}$.

Справедливо и обратное утверждение.

Теорема 2 (теорема Бёрлинга-Альфорса). Пусть $f: \mathbb{R} \rightarrow \mathbb{R}$ есть $k$-квазисимметричный гомеоморфизм. Тогда найдется квазиконформный гомеоморфизм $w: H \rightarrow H$, граничное значение которого совпадает $c f$, а максимальная дилатация ограничена числом $K(k)$, которое можно выбрать так, чтобы $K(k) \rightarrow 1$ nрu $k \rightarrow 1$.

Приведем идею доказательства этого результата (полное доказательство см. в [9]).

Определим отображение $w$, заданное на замыкании $\bar{H}$, по формуле

$$
w(x+i y)=\frac{1}{2} \int_{0}^{1}[f(x+t y)+f(x-i y)] d t+\frac{i}{2} \int_{0}^{1}[f(x+t y)-f(x-i y)] d t .
$$


Очевидно, что на вещественной оси $w(x)=f(x)$. Если ввести обозначения

$$
\begin{aligned}
& \alpha(x, y)=\int_{0}^{1} f(x+t y) d t=\frac{1}{y} \int_{x}^{x+y} f(t) d t \\
& \beta(x, y)=\int_{0}^{1} f(x-t y) d t=\frac{1}{y} \int_{x-y}^{x} f(t) d t
\end{aligned}
$$

то формула для $w$ запишется в виде

$$
w(x+i y)=\frac{\alpha+\beta}{2}+i \frac{\alpha-\beta}{2} .
$$

Геометрический смысл функций $\alpha$ и $\beta$ очевиден: функция $\alpha(x, y)$ сопоставляет точке $x+i y \in H$ среднее значение функции $f$ на отрезке $[x, x+y]$, а функция $\beta(x, y)$ - среднее значение $f$ на отрезке $[x-y, x]$.

Функции $\alpha$ и $\beta$ непрерывно дифференцируемы по $x$ и $y$, и определяемое ими отображение $w: H \rightarrow H$ имеет положительный якобиан (вычисленный в [9]). Из этого факта и того, что граничное значение $w$, совпадающее с $f$, является монотонно возрастающим гомеоморфизмом $\mathbb{R} \rightarrow \mathbb{R}$, следует, что $w$ задает гомеоморфизм $H \rightarrow H$. Условие квазиконформности $w$ переписывается в терминах неравенства, связывающего значения функции $f$ в точках $x, x+y$ и $x-y$, выполнение которого обеспечивается условием (13).

В случае единичного круга $\Delta$ условие квазисимметричности Бёрлинга-Альфорса (13) удобно формулировать в терминах перекрестного отношения. Напомним, что перекрестным (или двойным) отношением четырех попарно различных точек $z_{1}, z_{2}, z_{3}, z_{4}$ на комплексной плоскости называется величина

$$
\mathrm{CR}\left(z_{1}, z_{2}, z_{3}, z_{4}\right):=\frac{z_{4}-z_{1}}{z_{4}-z_{2}}: \frac{z_{3}-z_{1}}{z_{3}-z_{2}} .
$$

Совпадение перекрестных отношений $\mathrm{CR}\left(z_{1}, z_{2}, z_{3}, z_{4}\right)=\mathrm{CR}\left(w_{1}, w_{2}, w_{3}, w_{4}\right)$ является необходимым и достаточным условием существования конформного преобразования комплексной плоскости, переводящего точки $\left(z_{1}, z_{2}, z_{3}, z_{4}\right)$ в точки $\left(w_{1}, w_{2}, w_{3}, w_{4}\right)$.

Назовем сохраняющий ориентацию гомеоморфизм $f$ единичной окружности $S^{1}$ на себя квазисимметричным, если при некотором $0<\epsilon<1$ он удовлетворяет условию

$$
\frac{1}{2}(1-\epsilon) \leqslant \mathrm{CR}\left(f\left(z_{1}\right), f\left(z_{2}\right), f\left(z_{3}\right), f\left(z_{4}\right)\right) \leqslant \frac{1}{2}(1+\epsilon)
$$

для любой четверки попарно различных точек $z_{1}, z_{2}, z_{3}, z_{4}$ на $S^{1}$ с перекрестным отношением $\operatorname{CR}\left(z_{1}, z_{2}, z_{3}, z_{4}\right)=1 / 2$.

Условие (14) является аналогом для единичного круга $\Delta$ условия Бёрлинга-Альфорса (13). Как и в случае верхней полуплоскости $H$, оно гарантирует квазиконформную продолжаемость квазисимметричного гомеоморфизма $f: S^{1} \rightarrow S^{1}$ внутрь $\Delta$.

Поскольку любой диффеоморфизм окружности $S^{1}$ на себя, сохраняющий ориентацию, очевидно, удовлетворяет условию (14), то он квазисимметричен, т. е. допускает продолжение до квазиконформного диффеоморфизма единичного круга $\Delta$. 
3.2. Определение универсального пространства Тейхмюллера. Так как квазиконформные гомеоморфизмы единичного круга $\Delta$ образуют группу относительно композиции, то и квазисимметричные гомеоморфизмы единичной окружности $S^{1}$ образуют группу относительно этой операции. Мы обозначаем ее через $\mathrm{QS}\left(S^{1}\right)$. Как было отмечено выше, группа $\mathrm{QS}\left(S^{1}\right)$ содержит группу $\operatorname{Diff}_{+}\left(S^{1}\right)$ диффеоморфизмов $S^{1}$, сохраняющих ориентацию. Тем самым, имеется цепочка вложений

$$
\operatorname{Möb}\left(S^{1}\right) \subset \operatorname{Diff}_{+}\left(S^{1}\right) \subset \mathrm{QS}\left(S^{1}\right) \subset \operatorname{Homeo}_{+}\left(S^{1}\right),
$$

где Ноmео $\left(S^{1}\right)$ обозначает группу гомеоморфизмов $S^{1}$, сохраняющих ориентацию, а $\operatorname{Möb}\left(S^{1}\right)$ - группу дробно-линейных автоморфизмов единичного круга $\Delta$, суженных на $S^{1}$.

ОПРЕДЕЛЕНИЕ 1. Пространство

$$
\mathscr{T}=\operatorname{QS}\left(S^{1}\right) / \operatorname{Möb}\left(S^{1}\right)
$$

называется универсалъным пространством Тейхмюллера.

Как уже отмечалось выше, понятие универсального пространства Тейхмюллера было введено Берсом [17] в 1968 г. Оно играет важнейшую роль в теории квазиконформных отображений и ее приложениях. В последнее время была выявлена его связь с теорией струн, подробно рассмотренная в обзоре Пеконена [85].

Пространство $\mathscr{T}$ можно отождествить с подпространством $\mathrm{QS}\left(S^{1}\right)$, состоящим из нормализованных квазисимметричных гомеоморфизмов окружности, оставляющих три точки окружности $S^{1}$ неподвижными. В качестве таких точек принято выбирать \pm 1 и $-i$.

Универсальное пространство Тейхмюллера $\mathscr{T}$ содержит в качестве подпространства пространство

$$
\mathscr{S}=\operatorname{Diff}_{+}\left(S^{1}\right) / \operatorname{Möb}\left(S^{1}\right),
$$

которое можно отождествить с пространством нормализованных квазисимметричных диффеоморфизмов окружности.

Поскольку понятие квазиконформности допускает определение в терминах дифференциалов Бельтрами, удобно иметь определение универсального пространства Тейхмюллера $\mathscr{T}$ непосредственно в терминах этих дифференциалов.

Обозначим пространство дифференциалов Бельтрами в единичном круге $\Delta$ через

$$
B(\Delta) \equiv B_{(-1,1)}(\Delta) .
$$

Его можно отождествить с единичным шаром в комплексном банаховом пространстве $L^{\infty}(\Delta)$.

Пусть $\mu \in B(\Delta)$ есть дифференциал Бельтрами в круге $\Delta$. Продолжим его до дифференциала Бельтрами $\hat{\mu}$ во всей расширенной комплексной плоскости $\widehat{\mathbb{C}}$ с помощью симметрии относительно окружности $S^{1}$, полагая

$$
\hat{\mu}\left(\frac{1}{z}\right):=\overline{\mu(z)} \frac{z^{2}}{\bar{z}^{2}} \quad \text { при } z \in \Delta .
$$


Применяя теорему существования квазиконформных отображений к уравнению Бельтрами с продолженным дифференциалом Бельтрами $\hat{\mu}$, найдем нормализованный квазиконформный гомеоморфизм $w_{\mu}$ расширенной комплексной плоскости $\widehat{\mathbb{C}}$ с комплексной дилатацией $\hat{\mu}$. В силу теоремы единственности этот гомеоморфизм $w_{\mu}$ должен быть симметричен относительно $S^{1}$ и, следовательно, отображать окружность $S^{1}$ в себя. Таким образом, мы можем сопоставить исходному дифференциалу Бельтрами $\mu$ нормализованный квазисимметричный гомеоморфизм окружности $w_{\mu}$ :

$$
\left.B(\Delta) \ni \mu \mapsto w_{\mu}\right|_{S^{1}} \in \mathscr{T} .
$$

Это отображение взаимно однозначно по модулю следующего отношения эквивалентности дифференциалов Бельтрами:

$$
\left.\left.\mu \sim \nu \Longleftrightarrow w_{\mu}\right|_{S^{1}} \equiv w_{\nu}\right|_{S^{1}}
$$

Следовательно, универсалъное пространство Тейхмюллера $\mathscr{T}$ можно отождествить с фактором

$$
\mathscr{T}=B(\Delta) / \sim
$$

По-другому заданный дифференциал Бельтрами $\mu \in B(\Delta)$ можно продолжить на расширенную комплексную плоскость $\widehat{\mathbb{C}}$ до дифференциала Бельтрами $\check{\mu}$, полагая:

$$
\check{\mu}(z) \equiv 0 \text { при } z \in \Delta_{-}:=\widehat{\mathbb{C}} \backslash \bar{\Delta} .
$$

Применяя теорему существования квазиконформных отображений к уравнению Бельтрами с продолженным дифференциалом Бельтрами $\check{\mu}$, получим квазиконформный гомеоморфизм $w^{\mu}$ расширенной комплексной плоскости $\widehat{\mathbb{C}} \mathrm{c}$ комплексной дилатацией $\check{\mu}$. Удобно нормализовать его условием фиксации точек $0,1, \infty$. Построенный нормализованный квазиконформный гомеоморфизм $w^{\mu}$ будет конформным на дополнении $\Delta_{-}$к замкнутому единичному кругу $\bar{\Delta}$.

Назовем квазикругом образ единичного круга $\Delta$ при квазиконформном отображении, а квазиокружностью образ единичной окружности $S^{1}$ при таком отображении. С учетом этого определения мы только что построили отображение

$$
B(\Delta) \ni \mu \mapsto \text { квазикруг } \Delta^{\mu}:=w^{\mu}(\Delta) \text { в } \widehat{\mathbb{C}} .
$$

Это отображение взаимно однозначно по модулю следующего отношения эквивалентности дифференциалов Бельтрами:

$$
\left.\left.\mu \approx \nu \Longleftrightarrow w^{\mu}\right|_{\Delta_{-}} \equiv w^{\nu}\right|_{\Delta_{-}} .
$$

Можно показать (см. [64], [65]), что введенные отношения эквивалентности дифференциалов Бельтрами совпадают, т. е.

$$
\mu \sim \nu \Longleftrightarrow \mu \approx \nu \text {. }
$$

Благодаря этому факту, получаем еще две интерпретации универсального пространства Тейхмюллера:

$$
\begin{aligned}
\mathscr{T}= & \{\text { пространство нормализованных квазикругов в } \widehat{\mathbb{C}}\} \\
= & \{\text { пространство нормализованных квазиконформных } \\
& \text { отображений } \left.\widehat{\mathbb{C}} \rightarrow \widehat{\mathbb{C}}, \text { конформных в круге } \Delta_{-}\right\} .
\end{aligned}
$$


Связь между двумя реализациями универсального пространства Тейхмюллера в виде пространства нормализованных квазисимметричных гомеоморфизмов окружности $S^{1}$ и пространства нормализованных квазикругов в $\widehat{\mathbb{C}}$ может быть установлена и непосредственным образом.

Для этого воспользуемся следующей задачей факторизации, представляющей самостоятельный интерес. Пусть $f$ - сохраняющий ориентацию гомеоморфизм окружности $S^{1}$ на себя. Требуется найти конформные отображения $w_{+}$ и $w_{-}$, заданные соответственно в круге $\Delta_{+}$и его внешности $\Delta_{-}$, такие, что

$$
f=w_{+}^{-1} \circ w_{-} \quad \text { на } S^{1} .
$$

Пара отображений $\left(w_{+}, w_{-}\right)$называется нормализованной, если отображения $w_{ \pm}$оставляют на месте точки $\pm 1,-i$.

ЛЕмма 1. Пусть $f$ есть нормализованный квазисимметричный гомеоморфизм окружности $S^{1}$ на себя. Тогда задача факторизачии (16) допускает единственное нормализованное решение.

Действительно, для данного гомеоморфизма $f$ по теореме Бёрлинга-Альфорса найдется нормализованный квазиконформный гомеоморфизм $w: \Delta_{+} \rightarrow$ $\Delta_{+}$такой, что $\left.w\right|_{S^{1}}=f$. Обозначим через $\mu$ его комплексную дилатацию. Продолжим $\mu$ на $\widehat{\mathbb{C}}$ нулем вне $\Delta_{+}$до дифференциала Бельтрами $\check{\mu}$ и обозначим через $\widetilde{w}^{\mu}$ квазиконформный гомеоморфизм $\widehat{\mathbb{C}}$, комплексная дилатация которого совпадает с $\check{\mu}$, оставляющий на месте точки $\pm 1,-i \in S^{1}$. Тогда отображения

$$
\begin{aligned}
& w_{+}:=\left.\widetilde{w}^{\mu}\right|_{\Delta_{+}} \circ w_{\mu}^{-1}: \Delta_{+} \rightarrow \text { квазикруг } \widetilde{\Delta}^{\mu}, \\
& w_{-}:=\left.\widetilde{w}^{\mu}\right|_{\Delta_{-}}: \Delta_{-} \rightarrow \text { квазикруг } \widetilde{\Delta}_{-}^{\mu}
\end{aligned}
$$

конформны соответственно в $\Delta_{+}$и $\Delta_{-}$(конформность $w_{+}$вытекает из того, что гомеоморфизмы $\widetilde{w}^{\mu}$ и $w_{\mu}$ имеют одну и ту же комплексную дилатацию $\mu$ в круге $\left.\Delta_{+}\right)$. Поэтому они задают нормализованное решение задачи факторизации (16).

Вернемся к интересующему нас соответствию

$$
\left\{\begin{array}{c}
\text { нормализованные квазисимметричные } \\
\text { гомеоморфизмы } S^{1}
\end{array}\right\} \leftrightarrow\left\{\begin{array}{c}
\text { нормализованные } \\
\text { квазикруги в } \widehat{\mathscr{C}}
\end{array}\right\} .
$$

Если $f$ - нормализованный квазисимметричный гомеоморфизм $S^{1} \rightarrow S^{1}$, то он допускает единственную нормализованную факторизацию вида

$$
f=w_{+}^{-1} \circ w_{-},
$$

где $w_{+}=\left.\widetilde{w}^{\mu}\right|_{\Delta} \circ w_{\mu}^{-1}$ и $w_{-}=\left.\widetilde{w}^{\mu}\right|_{\Delta_{-}}$. Сопоставим ему нормализованный квазикруг $\Delta^{\mu}=w^{\mu}(\Delta)$.

Обратно, если $\Delta^{\mu}$ есть нормализованный квазикруг, отвечающий квазиконформному отображению $w^{\mu}$ с комплексной дилатацией $\mu$, то мы рассмотрим отображения

$$
w_{+}=\widetilde{w}^{\mu} \circ w_{\mu}^{-1} \quad \text { на } \Delta_{+} \quad \text { и } \quad w_{-}=\widetilde{w}^{\mu} \text { на } \Delta_{-} .
$$

Эти отображения конформны и оставляют на месте точки $\pm 1,-i$ на $S^{1}$. Сопоставим им квазисимметричный гомеоморфизм окружности $S^{1}$ на себя, задаваемый формулой

$$
f=w_{+}^{-1} \circ w_{-} \text {на } S^{1} .
$$

Это и есть искомый нормализованный квазисимметричный гомеоморфизм $S^{1} \rightarrow S^{1}$. 
3.3. Свойства универсального пространства Тейхмюллера. В этом пункте мы приводим основные свойства универсального пространства Тейхмюллера. Более подробное изложение свойств этого пространства можно найти в монографиях Лехто [66] и Нага [71].

Начнем с описания топологических свойств пространства $\mathscr{T}$ относительно топологии, задаваемой метрикой Тейхмюллера (доказательства этих свойств можно найти в книге [66]):

1) пространство $\mathscr{T}$ линейно связно;

2) пространство $\mathscr{T}$ полно, т. е. любая последовательность Коши в $\mathscr{T}$ сходится;

3) пространство $\mathscr{T}$ стягиваемо;

4) пространство $\mathscr{T}$ не является топологической группой; иными словами, операция композиции нормализованных квазисимметричных гомеоморфизмов $S^{1} \rightarrow S^{1}$ не является непрерывной в метрике Тейхмюллера.

Для того чтобы ввести на универсальном пространстве Тейхмюллера $\mathscr{T}$ комплексную структуру, построим его вложение в пространство голоморфных квадратичных дифференциалов в круге.

Пусть точке $[\mu] \in \mathscr{T}$ отвечает нормализованный квазиконформный гомеоморфизм $w^{\mu}$. Тогда $w^{\mu}$ конформен во внешности $\Delta_{-}$единичного круга $\Delta$, поэтому мы можем рассмотреть его производную Шварца

$$
S\left[\left.w^{\mu}\right|_{\Delta_{-}}\right]
$$

Полученная функция на $\Delta_{-}$не зависит от выбора $\mu \in[\mu]$ и голоморфна по $z \in \Delta_{-}$. Более того, при конформных заменах координат она преобразуется как квадратичный дифференциал. Отображение $[\mu] \mapsto S\left[\left.w^{\mu}\right|_{\Delta_{-}}\right]$является вложением, поскольку из равенства

$$
S\left[\left.w^{\mu}\right|_{\Delta_{-}}\right]=S\left[\left.w^{\nu}\right|_{\Delta_{-}}\right]
$$

следует, что $\left.w^{\mu}\right|_{\Delta_{-}}=\left.w^{\nu}\right|_{\Delta_{-}}$, т. е. $\mu \sim \nu$.

Итак, мы построили вложение

$$
\Psi: \mathscr{T} \rightarrow B_{2}\left(\Delta_{-}\right)
$$

универсального пространства Тейхмюллера $\mathscr{T}$ в пространство $B_{2}\left(\Delta_{-}\right)$голоморфных квадратичных дифференциалов в круге $\Delta_{-}$, называемое вложением Берса. Множество $B_{2}\left(\Delta_{-}\right)$является комплексным банаховым пространством, наделенным естественной гиперболической нормой:

$$
B_{2}\left(\Delta_{-}\right)=\left\{\psi=\psi(z) d z^{2}:\|\psi\|_{B_{2}}:=\sup _{z \in \Delta_{-}}\left(1-|z|^{2}\right)^{2}|\psi(z)|<\infty\right\} .
$$

Вложение $\Psi$ является гомеоморфизмом пространства $\mathscr{T}$ на его образ в $B_{2}\left(\Delta_{-}\right)$, описываемый следующей теоремой.

Теорема 3. Образ $\Psi(\mathscr{T})$ в $B_{2}\left(\Delta_{-}\right)$является связным открытым стягиваемым подмножеством в $B_{2}\left(\Delta_{-}\right)$, которое содержит открытый шар $B(0,2)$ радиуса 2 с иентром в начале и содержится в замкнутом шаре $B(0,6)$.

Доказательство этой теоремы можно найти в книге [66]. 
Введем комплексную структуру на пространстве $\mathscr{T}$, индуцированную комплексной структурой на комплексном банаховом пространстве $B_{2}\left(\Delta_{-}\right)$посредством вложения Берса.

По-другому на $\mathscr{T}$ можно было бы ввести комплексную структуру, индуцированную естественной проекцией

$$
B(\Delta) \rightarrow \mathscr{T}=B(\Delta) / \sim
$$

Оказывается, оба способа дают один и тот же результат, точнее, имеет место следующая

Теорема 4 [71]. Композиция естественной проекции $B(\Delta) \rightarrow \mathscr{T}$ с вложением Берса, задаюшая отображение

$$
F: B(\Delta) \rightarrow B_{2}\left(\Delta_{-}\right),
$$

является голоморфным отображением комплексных банаховых пространств.

Дадим явное описание касательного отображения $d F$ в начале, т. е. в точке $\mu \equiv 0$. Эта точка при отображении $F$ переходит в класс $[\mathrm{id}]=\left[\operatorname{Möb}\left(S^{1}\right)\right]$ в представлении

$$
\mathscr{T}=\mathrm{QS}\left(S^{1}\right) / \operatorname{Möb}\left(S^{1}\right) \text {. }
$$

Пусть $\mu \in L^{\infty}(\Delta)$ есть произвольный касательный вектор из пространства $T_{0} B(\Delta)$. Тогда при достаточно малом $t$ функция $t \mu$ принадлежит $B(\Delta)$ и потому определяет отвечающий ей нормализованный квазиконформный гомеоморфизм $w^{t \mu}$, для которого справедливо представление

$$
w^{t \mu}(z)=z+t w_{1}(z)+o(t)
$$

при $t \rightarrow 0$, где $o(t) \equiv t \varepsilon(z, t)$ и $\varepsilon(z, t) \rightarrow 0$ равномерно по $z$, принадлежащим компактным подмножествам в $\mathbb{C}$.

Коэффициент

$$
w_{1}(z) \equiv \dot{w}[\mu](z)
$$

представляет собой первую вариацию квазиконформного гомеоморфизма $w^{\mu}$ относительно $\mu$.

Подставим $w^{t \mu}$ в уравнение Бельтрами и продифференцируем полученное соотношение по $t$ при $t=0$. В результате получим соотношение

$$
\bar{\partial} w^{t \mu}=t \mu \partial w^{t \mu}
$$

из которого следует, что

$$
\left.\frac{\partial}{\partial t}\right|_{t=0}\left(\bar{\partial} w^{t \mu}\right)=\left.\mu\left(\partial w^{t \mu}\right)\right|_{t=0} .
$$

Из представления (20) вытекает, что

$$
\left.\partial w^{t \mu}\right|_{t=0}=1,\left.\quad \frac{\partial}{\partial t}\right|_{t=0} w^{t \mu}=w_{1}(z),
$$

поэтому из соотношения (22) получаем $\bar{\partial}$-уравнение на функцию $w_{1}(z)$ :

$$
\bar{\partial} w_{1}=\mu,
$$


которое выполняется для почти всех $z \in \mathbb{C}$. Если носитель $\mu$ компактен, то решение этого уравнения задается интегралом Коши-Грина

$$
-\frac{1}{\pi} \int_{\mathbb{C}} \frac{\mu(\zeta)}{\zeta-z} d \xi d \eta, \quad \zeta=\xi+i \eta,
$$

плюс произвольная целая функция. Можно показать (см. [9]), что эта целая функция может быть только линейной вида $A+B z$. Постоянные $A$ и $B$ нетрудно найти из условий нормализации

$$
w^{t \mu}(0, t) \equiv 0, \quad w^{t \mu}(1, t) \equiv 1,
$$

откуда $w_{1}(0)=w_{1}(1)=0$. Из последних соотношений следует, что

так что

$$
A=\frac{1}{\pi} \int_{\mathbb{C}} \frac{\mu(\zeta)}{\zeta} d \xi d \eta, \quad B=\frac{1}{\pi} \int_{\mathbb{C}} \mu(\zeta)\left[\frac{1}{\zeta-1}-\frac{1}{\zeta}\right] d \xi d \eta,
$$

$$
A+B z=\frac{1-z}{\pi} \int_{\mathbb{C}} \frac{\mu(\zeta)}{\zeta} d \xi d \eta+\frac{z}{\pi} \int_{\mathbb{C}} \frac{\mu(\zeta)}{\zeta-1} d \xi d \eta .
$$

Следовательно, искомое решение уравнения (23) задается формулой

$$
\begin{aligned}
w_{1}(z) & =-\frac{1}{\pi} \int_{\mathbb{C}} \mu(\zeta)\left[\frac{1}{\zeta-z}+\frac{z-1}{\zeta}-\frac{z}{\zeta-1}\right] d \xi d \eta \\
& =-\frac{z(z-1)}{\pi} \int_{\mathbb{C}} \frac{\mu(\zeta) d \xi d \eta}{\zeta(\zeta-1)(\zeta-z)} .
\end{aligned}
$$

(Эта формула получена в предположении, что $\mu$ имеет компактный носитель в $\mathbb{C}$. Последнее ограничение можно снять с помощью рассуждения из [9].)

Пользуясь формулой (24), можно доказать следующую теорему.

Теорема 5 [70]. Дифференциал отображения

$$
F: B(\Delta) \rightarrow B_{2}\left(\Delta_{-}\right)
$$

в точке $\mu \equiv 0$ является ограниченным линейным оператором $d_{0} F: L^{\infty}(\Delta) \rightarrow$ $B_{2}\left(\Delta_{-}\right)$, задаваемым формулой

$$
d_{0} F[\mu](z)=-\frac{6}{\pi} \int_{\Delta} \frac{\mu(\zeta)}{(\zeta-z)^{4}} d \xi d \eta, \quad z \in \Delta_{-}, \quad \zeta=\xi+i \eta \in \Delta .
$$

Операторная норма $d_{0} F$ оценивается абсолютной константой.

Приведем идею доказательства этой теоремы. Фиксируем точку $z_{0} \in \Delta_{-}$. Можно показать, что функция $w^{t \mu}(z)$ является голоморфной как по $z$, так и по $t$ (и, следовательно, по их совокупности) при достаточно малых $|t|$ и $\left|z-z_{0}\right|$. Поэтому функция

$$
\varphi(t, z):=S\left[w^{t \mu}\right](z),
$$

являющаяся образом функции $t \mu$ при отображении Берса, голоморфна в области

$$
\{|t|<\epsilon\} \times\left\{\left|z-z_{0}\right|<\delta\right\} \subset \mathbb{C}^{2}
$$

при достаточно малых $\epsilon$ и $\delta$. Вычислим производную этой функции по $t$ при $t=0$. Для сокращения формул будем обозначать производную по $t$ "точкой", а производную по $z$ "штрихом". Тогда

$$
\dot{\varphi}=\left[\frac{w^{\prime \prime \prime}}{w^{\prime}}-\frac{3}{2} \frac{\left(w^{\prime \prime}\right)^{2}}{\left(w^{\prime}\right)^{2}}\right]^{\cdot}=\frac{\left(w^{\prime}\right)^{3} \dot{w}^{\prime \prime \prime}-\dot{w}^{\prime}\left(w^{\prime}\right)^{2} w^{\prime \prime \prime}-3 \dot{w}^{\prime \prime}\left(w^{\prime}\right)^{2} w^{\prime \prime}+6 \dot{w}^{\prime} w^{\prime} w^{\prime \prime}}{\left(w^{\prime}\right)^{4}} .
$$


При $t=0$ имеем $w(z) \equiv z$, откуда следует, что $w^{\prime} \equiv 1, w^{\prime \prime}=w^{\prime \prime \prime} \equiv 0$. Поэтому

$$
\left.\frac{\partial \varphi}{\partial t}\right|_{t=0}=\frac{\left(w^{\prime}\right)^{3} \dot{w}^{\prime \prime \prime}}{\left(w^{\prime}\right)^{4}}=\dot{w}^{\prime \prime \prime} .
$$

Теперь воспользуемся формулой (24) для функции $w_{1}(z)=\dot{w}[\mu](z)$ :

$$
\dot{w}(z)=-\frac{z(z-1)}{\pi} \int_{\Delta} \frac{\mu(\zeta) d \xi d \eta}{\zeta(\zeta-1)(\zeta-z)}, \quad z \in \mathbb{C} .
$$

Поскольку интеграл в последней формуле равномерно ограничен по $z$ на компактных подмножествах из $\Delta_{-}$, мы можем продифференцировать по $z$ под знаком интеграла. В итоге получим

$$
\dot{w}^{\prime \prime \prime}(z)=-\frac{6}{\pi} \int_{\Delta} \frac{\mu(\zeta)}{(\zeta-z)^{4}} d \xi d \eta, \quad z \in \Delta_{-} .
$$

Оценка нормы оператора $d_{0} F$ вытекает из оценки интеграла

$$
\int_{\Delta} \frac{|d \xi d \eta|}{|\zeta-z|^{4}} \leqslant \frac{\pi}{\operatorname{dist}\left(z, S^{1}\right)^{2}} \quad \text { при } z \in \Delta_{-} .
$$

Опишем теперь ядро дифференциала $d_{0} F$. Для этого введем подпространство $A_{2}(\Delta) \subset B_{2}(\Delta)$, состоящее из суммируемых (т. е. $L^{1}$-интегрируемых) голоморфных квадратичных дифференциалов в круге $\Delta$ :

$$
A_{2}(\Delta)=\left\{\psi=\psi(z) d z^{2} \in B_{2}(\Delta): \int_{\Delta}|\psi(z)||d x d y|<\infty\right\} .
$$

Между этим пространством и пространством $B(\Delta)$ дифференциалов Бельтрами имеется естественное спаривание

$$
\langle\mu, \psi\rangle=\int_{\Delta} \mu \psi
$$

Это обычное спаривание между $(-1,1)$ - и $(2,0)$-дифференциалами, при котором интеграл в правой части берется от интегрируемой $(1,1)$-формы.

$\mathrm{B}$ терминах указанного спаривания ядро $d_{0} F$ описывается следующим образом.

Лемма 2 (лемма Тейхмюллера, см. [9]). Ядро дифференциала $d_{0} F$ совпадает с подпространством

$$
N:=A_{2}(\Delta)^{\perp}=\left\{\mu \in L^{\infty}(\Delta):\langle\mu, \psi\rangle=0 \text { для всех } \psi \in A_{2}(\Delta)\right\} .
$$

Пользуясь приведенными результатами, попытаемся ввести кэлерову метрику на пространстве $\mathscr{T}$. Для этого воспользуемся отображением Альфорса

$$
\Phi: L^{\infty}(\Delta) \rightarrow B_{2}(\Delta),
$$

сопоставляющим функции $\mu \in L^{\infty}(\Delta)$ интеграл

$$
\Phi[\mu](z) \equiv \varphi(z)=\int_{\Delta} \frac{\overline{\mu(\zeta)}}{(1-z \bar{\zeta})^{4}} d \xi d \eta .
$$

Образом функции $\mu$ при этом отображении является голоморфный квадратичный дифференциал $\varphi=\varphi(z) d z^{2}$ в круге $\Delta$. Ядро отображения $\Phi$ совпадает c $N=A_{2}(\Delta)^{\perp}$. 
Нам хотелось бы определить эрмитову метрику на пространстве $\mathscr{T}$, пользуясь формулой (25). Попробуем сначала определить ее в нуле, чтобы затем разнести в другие точки $\mathscr{T}$ с помощью действия группы $\mathrm{QS}\left(S^{1}\right)$ на $\mathscr{T}$ левыми сдвигами. Эрмитову метрику на касательном пространстве $T_{0} \mathscr{T}$ естественно определять, полагая ее равной на касательных векторах $[\mu],[\nu] \in T_{0} \mathscr{T}=L^{\infty}(\Delta) / N$ двойному интегралу

$$
(\mu, \nu) \equiv\langle\mu, \Phi[\nu]\rangle=\int_{\Delta} \int_{\Delta} \frac{\mu(z) \overline{\nu(\zeta)}}{(1-z \zeta)^{4}} d \xi d \eta d x d y .
$$

Однако введенная таким образом метрика оказывается корректно определенной только на плотном подмножестве в $T_{0} \mathscr{T}$. Причина в том, что для общего $\nu \in L^{\infty}(\Delta)$ его образ $\Phi[\nu]$ в $B_{2}(\Delta)$ может оказаться не интегрируемым, т. е. не принадлежащим $A_{2}(\Delta)$, и в этом случае интеграл в формуле (26) разойдется. На самом деле, формула (26) корректно определена только для достаточно гладких касательных векторов $[\mu],[\nu]$ из $T_{0} \mathscr{T}$.

Сформулируем это утверждение более точно. Пусть $[\mu] \in L^{\infty}(\Delta)$ есть касательный вектор из $T_{0} \mathscr{T}$. Рассмотрим отображение

$$
d \beta: T_{0}(B(\Delta) / \sim) \rightarrow T_{[\mathrm{id}]}\left(\mathrm{QS}\left(S^{1}\right) / \operatorname{Möb}\left(S^{1}\right)\right),
$$

касательное к изоморфизму

$$
\beta: B(\Delta) / \sim \rightarrow \mathrm{QS}\left(S^{1}\right) / \operatorname{Möb}\left(S^{1}\right) .
$$

При этом отображении касательный вектор $[\mu]$ переходит в некоторое векторное поле на $S^{1}$ вида

$$
v(\theta) \frac{d}{d \theta}=\dot{w}[\mu](z) \frac{d}{d z}, \quad z=e^{i \theta},
$$

где $\dot{w}[\mu]$ - первая вариация квазиконформного гомеоморфизма $w^{\mu}$ по $\mu$ из формулы (21). Будем называть векторные поля на $S^{1}$, являющиеся образами элементов $[\mu] \in T_{0} \mathscr{T}$ при отображении $d \beta$, квазисимметричными. Ниже будет показано, что интеграл в формуле (26) сходится, если векторам $\mu, \nu$ отвечают векторные поля $\dot{w}[\mu], \dot{w}[\nu]$ на $S^{1}$ с гладкостью класса $C^{3 / 2+\epsilon}$ с любым $\epsilon>0$.

Несмотря на то что формула (26) задает только плотно заданную квазиметрику на пространстве $\mathscr{T}$, ее сужение на классические пространства Тейхмюллера $T(G)$ и на пространство нормализованных диффеоморфизмов $\mathscr{S}$ оказывается уже корректно определенной кэлеровой метрикой (мы обсудим этот вопрос более подробно в следующем пункте).

Вопросу о существовании кэлеровой метрики на универсальном пространстве Тейхмюллера посвящено большое количество статей. Отметим в этой связи монографию Тахтаджяна и Тео [104], где такая метрика построена, однако топология, индуцируемая ею, отличается от топологии, индуцируемой метрикой Тейхмюллера. (В частности, универсальное пространство Тейхмюллера в этой топологии распадается на несчетное множество связных компонент.)

Вернемся к соответствию (27), рассмотренному выше, и дадим внутреннее описание квазисимметричных векторных полей на $S^{1}$, являющихся образами векторов $[\mu] \in T_{0}(B(\Delta) / \sim)$ при отображении (27). Поскольку в этом описании существенную роль играет условие Бёрлинга-Альфорса, удобно начать со случая верхней полуплоскости $H$. 
Введем пространство Зигмунда $\Lambda(\mathbb{R})$, состоящее из непрерывных функций $f: \mathbb{R} \rightarrow \mathbb{R}$, удовлетворяющих условиям

$$
f(0)=f(1)=0, \quad \frac{f(x)}{x^{2}+1} \rightarrow 0 \quad \text { при } x \rightarrow \infty,
$$

для которых существует константа $C>0$ такая, что

$$
|f(x+t)+f(x-t)-2 f(x)| \leqslant C|t| \quad \text { при всех } x \in \mathbb{R}, t>0 .
$$

Пространство $\Lambda(\mathbb{R})$ является (не сепарабельным) банаховым пространством с нормой

$$
\|f\|_{\Lambda}:=\sup _{x, t}\left|\frac{f(x+t)+f(x-t)-2 f(x)}{t}\right| .
$$

В работе [34] показано, что квазисимметричные векторные поля на $\mathbb{R}$ отвечают в точности функциям из пространства Зигмунда $\Lambda(\mathbb{R})$.

Отсюда нетрудно получить аналог пространства $\Lambda(\mathbb{R})$ для окружности $S^{1}$, пользуясь преобразованием Кэли.

3.4. Подпространства универсального пространства Тейхмюллера. Классические пространства Тейхмюллера $T(G)$, где $G \subset \operatorname{Möb}\left(S^{1}\right)$ - фуксова группа, вкладываются в универсальное пространство Тейхмюллера в виде комплексных подмногообразий.

Напомним, что квазисимметричный гомеоморфизм $f \in \operatorname{QS}\left(S^{1}\right)$ называется $G$-инвариантным относительно фуксовой группы $G$, если

$$
f G f^{-1} \subset \operatorname{Möb}\left(S^{1}\right) .
$$

Обозначим подгруппу $G$-инвариантных квазисимметричных гомеоморфизмов в $\operatorname{QS}\left(S^{1}\right)$ через $\operatorname{QS}\left(S^{1}, G\right)$ и определим классическое пространство Тейхмюллера $T(G)$ как

$$
T(G)=\mathrm{QS}\left(S^{1}, G\right) / \operatorname{Möb}\left(S^{1}\right) .
$$

Пространство Тейхмюллера $T(G)$ есть комплексное банахово многообразие, комплексная структура которого индуцируется вложением Берса или естественной проекцией пространства $G$-инвариантных дифференциалов Бельтрами $B(\Delta, G) \rightarrow T(G)=B(\Delta, G) / \sim$. Вложение $B(\Delta, G) \rightarrow B(\Delta)$ порождает вложение пространства Тейхмюллера $T(G)$ в универсальное пространство Тейхмюллера $\mathscr{T}$, отвечающее фуксовой группе $\{1\}$, в виде комплексного подмногообразия.

Пусть риманова поверхность $S_{0}$ униформизуется фуксовой группой $G$, т. е.

$$
S_{0}=\Delta / G \text {. }
$$

По каждому классу $[\mu] \in T(G)$ мы можем построить новую риманову поверхность

$$
S_{\mu}=\Delta / G_{\mu},
$$

где $G_{\mu}=w_{\mu} G w_{\mu}^{-1}$. Эту же поверхность можно записать в виде

$$
S_{\mu}=\Delta^{\mu} / G^{\mu},
$$


где $\Delta^{\mu}:=w^{\mu}(\Delta), G^{\mu}=w^{\mu} G\left(w^{\mu}\right)^{-1}$. Поверхности $S_{\mu}=\Delta^{\mu} / G^{\mu}$ гомеоморфны друг другу и различаются только своими комплексными структурами. В то же время риманова поверхность $\Delta_{-}^{\mu} / G^{\mu}$, где $\Delta_{-}^{\mu}:=w^{\mu}\left(\Delta_{-}\right)$, биголоморфно эквивалентна $\Delta_{-} / G$, поскольку $w^{\mu}$ конформно на $\Delta_{-}$.

Иными словами, пространство $T(G)$ параметризует, посредством сопоставления $[\mu] \mapsto G_{\mu}$, различные комплексные структуры на римановой поверхности $S_{0}=\Delta / G$, получающиеся из исходной квазиконформными деформациями.

Все свойства универсального пространства Тейхмюллера, изложенные выше, переносятся и на классические пространства Тейхмюллера, нужно только добавлять всюду условие $G$-инвариантности.

Так, вложение Берса в случае единичного круга $\Delta$ задается отображением

$$
F: B(\Delta, G) \rightarrow B_{2}\left(\Delta_{-}, G\right),
$$

сопоставляющим дифференциалу Бельтрами $\mu \in B(\Delta, G)$ голоморфный квадратичный дифференциал $S\left[\left.w^{\mu}\right|_{\Delta_{-}}\right]$на $\Delta_{-}$. Пространство $B_{2}\left(\Delta_{-}, G\right)$ состоит по определению из $G$-инвариантных голоморфных квадратичных дифференциалов в $\Delta_{-}$, имеющих конечную норму

$$
\|\psi\|_{2}:=\sup _{z \in \Delta_{-}}\left(1-|z|^{2}\right)^{2}|\psi(z)|<\infty .
$$

Формула для дифференциала $d_{0} F$ имеет тот же вид:

$$
d_{0} F[\mu](z)=-\frac{6}{\pi} \int_{\Delta} \frac{\mu(\zeta)}{(\zeta-z)^{4}} d \xi d \eta, \quad z \in \Delta_{-},
$$

для $\mu \in L^{\infty}(\Delta, G)$. Ядро $d_{0} F$ совпадает с подпространством

$$
N(G) \equiv A_{2}(\Delta, G)^{\perp}=\left\{\mu \in L^{\infty}(\Delta, G):\langle\mu, \psi\rangle=0 \text { для всех } \psi \in A_{2}(\Delta, G)\right\} .
$$

Таким образом, касательное пространство к $T(G)$ в начале совпадает с $L^{\infty}(\Delta, G) / N(G)$.

Как и в случае универсального пространства Тейхмюллера, имеется отображение Альфорса $L^{\infty}(\Delta, G) / N(G) \rightarrow B_{2}(\Delta, G)$, задаваемое формулой

$$
L^{\infty}(\Delta, G) \ni \mu \mapsto \Phi[\mu](z)=\int_{\Delta} \frac{\overline{\mu(\zeta)}}{(1-z \bar{\zeta})^{4}} d \xi d \eta .
$$

Попытаемся, как и выше, ввести с помощью этого отображения кэлерову метрику на пространстве $T(G)$, задавая ее на векторах $[\mu],[\nu]$ из

$$
T_{0} T(G)=L^{\infty}(\Delta, G) / N(G)
$$

формулой

$$
(\mu, \nu)_{G}=\langle\mu, \Phi[\nu]\rangle_{G}:=\int_{\Delta / G} \int_{\Delta} \frac{\mu(z) \overline{\nu(\zeta)}}{(1-z \bar{\zeta})^{4}} d \xi d \eta d x d y
$$

В случае классических пространств Тейхмюллера $T(G)$ пространство $B_{2}(\Delta, G)$ совпадает с пространством интегрируемых голоморфных квадратичных дифференциалов $A_{2}(\Delta, G)$ (см. [71]), поэтому спаривание (28) корректно определено для всех $\mu, \nu \in L^{\infty}(\Delta, G)$ и задает эрмитову метрику на пространстве $T_{0} T(G)$, называемую метрикой Вейля-Петерсона. Указанная кэлерова метрика на 
пространствах $T(G)$ была введена и исследована в работах А. Вейля [120] и Л. Альфорса [8]. В частности, Альфорс показал, что она имеет отрицательную голоморфную секционную кривизну. Подробное исследование свойств этой метрики и ее геодезических проведено в работах Вольперта (см. [122], [123], [125]). Интересная связь между указанной метрикой и уравнением Лиувилля установлена в работах Зографа и Тахтаджяна (см. [127], [128]).

Что можно сказать об образе классических пространств $T(G)$ в универсальном пространстве Тейхмюллера $\mathscr{T} ?$ Имеется любопытный результат Боуэна [21], показывающий, что этот образ не принадлежит регулярной части $\mathscr{T}$.

Более точно, будем называть точку из $\mathscr{T}$ регулярной, если ей отвечает гладкий нормализованный квазисимметричный гомеоморфизм из $\operatorname{QS}\left(S^{1}\right)$ или, что то же самое, квазикруг с гладкой границей. Боуэн показал, что каждой точке из $T(G) \backslash\{0\}$ отвечает квазикруг с фрактальной границей, хаусдорфова размерность $d_{H}$ которой находится в пределах $1<d_{H}<2$ и может быть любым числом из этого промежутка. В терминах квазисимметричных гомеоморфизмов можно доказать, что если $f$ есть $G$-инвариантный квазисимметричный гомеоморфизм, принадлежащий классу $C^{1}$ хотя бы в одной точке, то $f \in \operatorname{Möb}\left(S^{1}\right)$.

Обратимся теперь к подпространству $\mathscr{T}$ иного рода, а именно к пространству нормализованных диффеоморфизмов

$$
\mathscr{S}=\operatorname{Diff}_{+}\left(S^{1}\right) / \operatorname{Möb}\left(S^{1}\right) \subset \mathscr{T}=\mathrm{QS}\left(S^{1}\right) / \operatorname{Möb}\left(S^{1}\right) \text {. }
$$

Оно целиком лежит в регулярной части пространства $\mathscr{T}$, и вложение (29) наделяет его индуцированной комплексной структурой. Однако эту структуру на $\mathscr{S}$ можно ввести и более непосредственным образом.

Прежде всего заметим, что указанную комплексную структуру достаточно определить в начале $[\mathrm{id}] \in \mathscr{S}$, а затем разнести в другие точки $\mathscr{S}$ с помощью действия группы Diff $+\left(S^{1}\right)$. Построенная таким образом комплексная структура будет, тем самым, Diff $+\left(S^{1}\right)$-инвариантной. Касательное пространство

$$
T_{\text {[id] }} \mathscr{S}=T_{\text {[id] }}\left(\operatorname{Diff}_{+}\left(S^{1}\right) / \operatorname{Möb}\left(S^{1}\right)\right)
$$

можно отождествить с фактором алгебры Ли $\operatorname{Diff}_{+}\left(S^{1}\right)$ по ее подалгебре $\mathrm{sl}(2, \mathbb{R})$, совпадающей с алгеброй Ли группы Ли $\operatorname{Möb}\left(S^{1}\right)$. Алгебра Ли группы Ли $\operatorname{Diff}_{+}\left(S^{1}\right)$ совпадает с алгеброй Ли $\operatorname{Vect}\left(S^{1}\right)$ гладких векторных полей на $S^{1}$, a ее элементы $v=v(\theta) \partial / \partial \theta$ удобно задавать разложениями Фурье вида

$$
v=\sum_{n \in \mathscr{Z}} v_{n} e_{n}
$$

с комплексными коэффициентами, удовлетворяющими условию $\bar{v}_{n}=v_{-n}$, где $e_{n}$ - базисные векторные поля

$$
e_{n}=e^{i n \theta} \frac{\partial}{\partial \theta}=i z^{n+1} \frac{\partial}{\partial z}, \quad n \in \mathbb{Z}, \quad z=e^{i \theta} .
$$

Тогда элементы $v \in T_{[\mathrm{id}]} \mathscr{S}$ будут задаваться рядами вида

$$
v=\sum_{n \neq 0, \pm 1} v_{n} e_{n}
$$

Комплексная структура $J$ на пространстве $T_{[\mathrm{id}]} \mathscr{S}$ определяется формулой

$$
J v=-i \sum_{n=2}^{\infty} v_{n} e_{n}+i \sum_{n=-\infty}^{-1} v_{n} e_{n} .
$$


Введенная таким образом комплексная структура эквивалентна построенной ранее комплексной структуре на пространстве $\mathscr{T}$ (см. [74]).

Пространство $\mathscr{S}$ обладает однородной симплектической формой $\omega$. Эта форма определяется однозначно с точностью до умножения на константу, и ее значения на базисных векторных полях $e_{n} \in T_{[\mathrm{id}]}^{\mathbb{C}} \mathscr{S}$ равны (см. [97]):

$$
\omega\left(e_{m}, e_{n}\right)=\alpha\left(m^{3}-m\right) \delta_{m,-n}, \quad m, n \in \mathbb{Z} \backslash\{0, \pm 1\}, \quad \alpha \in \mathbb{C} \backslash\{0\} .
$$

По форме $\omega$ и комплексной структуре $J$ можно построить согласованную с ними риманову метрику $g_{\mathrm{R}}$, задаваемую на касательных векторах $u, v \in T_{\text {[id] }} \mathscr{S}$ формулой

$$
g_{\mathrm{R}}(u, v)=a \operatorname{Re}\left[\sum_{n=2}^{\infty} \bar{u}_{n} v_{n}\left(n^{3}-n\right)\right], \quad a \in \mathbb{R} \backslash\{0\},
$$

где

$$
u=\sum_{n \neq 0, \pm 1} u_{n} e_{n}, \quad v=\sum_{n \neq 0, \pm 1} v_{n} e_{n}
$$

Кэлерова метрика

$$
g(u, v)=a \sum_{n=2}^{\infty} \bar{u}_{n} v_{n}\left(n^{3}-n\right)
$$

является комплексификацией построенной римановой метрики $g_{\mathrm{R}}$. Заметим, что ряд в правой части (31) абсолютно сходится, если векторные поля $u, v$ принадлежат классу $C^{3 / 2+\epsilon}$ с любым $\epsilon>0$.

Кэлерова метрика (31) на пространстве $\mathscr{S}$ была построена в работе Кириллова и Юрьева [54] (см. также [22]). Она совпадает с ограничением на $\mathscr{S}$ кэлеровой квазиметрики (26) при подходящем выборе константы $a$. Точнее, имеет место следующее предложение.

ПреДЛОЖЕНИЕ 7 [74]. Пусть векторы $\mu, \nu \in L^{\infty}(\Delta)$ отвечают $C^{3 / 2+\epsilon}$-гладким векторным полям $u, v$ на $S^{1}$ соответственно. Тогда значение метрики (31) на этих полях равно

$$
g(u, v)=\frac{a}{6 \pi^{2}} \int_{\Delta} \int_{\Delta} \frac{\mu(z) \overline{\nu(\zeta)}}{(1-z \bar{\zeta})^{4}} d x d y
$$

Образ вложения $\mathscr{S} \hookrightarrow \mathscr{T}$ лежит, как указывалось ранее, в регулярной части пространства $\mathscr{T}$. С другой стороны, образы вложений классических пространств Тейхмюллера $T(G) \backslash\{0\}$ принадлежат нерегулярной части $\mathscr{T}$. Тем самым, эти подмногообразия пересекаются только в начале $[\mathrm{id}] \in \mathscr{T}$.

Подробное изложение свойств пространства нормализованных диффеморфизмов $\mathscr{S}$ можно найти в книге [97] и цитированных там статьях.

3.5. Грассманова реализация универсального пространства Тейхмюллера. В этом пункте строится грассманова реализация универсального пространства Тейхмюллера, задаваемая вложением пространства $\mathscr{T}$ в грассманиан Гильберта-Шмидта, состоящий из бесконечномерных подпространств соболевского гильбертова пространства полудифференцируемых функций на окружности. Существование указанного вложения вытекает из теоремы Нага-Сулливана, играющей ключевую роль в квантовании универсального пространства Тейхмюллера. Эта теорема доказана в статье [73], где сообщаются 
и другие факты, относящиеся к вложению универсального пространства Тейхмюллера в бесконечномерный грассманиан (см. также [90] и [97]).

Соболевским пространством полудифферениируемых функиий называется гильбертово пространство

$$
V=H_{0}^{1 / 2}\left(S^{1}, \mathbb{R}\right)
$$

состоящее из функций $f \in L^{2}\left(S^{1}, \mathbb{R}\right)$ с нулевым средним по окружности, имеющих обобщенную производную порядка $1 / 2$ из $L^{2}\left(S^{1}, \mathbb{R}\right)$. Иначе говоря, $V$ состоит из функций $f \in L^{2}\left(S^{1}, \mathbb{R}\right)$, ряды Фурье которых имеют вид

$$
f(z)=\sum_{n \neq 0} f_{n} z^{n}, \quad \bar{f}_{n}=f_{-n}, \quad z=e^{i \theta},
$$

с конечной соболевской нормой порядка $1 / 2$ :

$$
\|f\|_{1 / 2}^{2}=\sum_{n \neq 0}|n|\left|f_{n}\right|^{2}=2 \sum_{n=1}^{\infty} n\left|f_{n}\right|^{2}<\infty .
$$

Рассмотрим на пространстве $V$ кососимметрическую 2-форму $\omega: V \times V \rightarrow \mathbb{R}$, которая определяется в терминах коэффициентов Фурье векторов $\xi, \eta \in V$ по формуле

$$
\omega(\xi, \eta)=-i \sum_{n \neq 0} n \xi_{n} \eta_{-n}=2 \operatorname{Im} \sum_{n=1}^{\infty} n \xi_{n} \bar{\eta}_{n} .
$$

Эта форма корректно определена ввиду неравенства Коши-Шварца

$$
|\omega(\xi, \eta)| \leqslant\|\xi\|_{1 / 2}\|\eta\|_{1 / 2} .
$$

Пространство $V$ обладает также комплексной структурой $J^{0}$, которая определяется в терминах разложений Фурье формулой

$$
\xi(z)=\sum_{n \neq 0} \xi_{n} z^{n} \mapsto\left(J^{0} \xi\right)(z)=-i \sum_{n=1}^{\infty} \xi_{n} z^{n}+i \sum_{n=-\infty}^{-1} \xi_{n} z^{n} .
$$

Эта комплексная структура совместима с симплектической формой $\omega$ в том смысле, что вместе они определяют риманову метрику на $V$ по формуле $g^{0}(\xi, \eta)$ $:=\omega\left(\xi, J^{0} \eta\right)$ или, в терминах коэффициентов Фурье,

$$
g^{0}(\xi, \eta)=\sum_{n \neq 0}|n| \xi_{n} \bar{\eta}_{n}=2 \operatorname{Re} \sum_{n=1}^{\infty} n \xi_{n} \bar{\eta}_{n} .
$$

Иными словами, $V$ является кэлеровым гильбертовым пространством.

Комплексификация

$$
V^{\mathbb{C}}=H_{0}^{1 / 2}\left(S^{1}, \mathbb{C}\right)
$$

пространства $V$ является комплексным гильбертовым пространством, состоящим из функций $f \in L^{2}\left(S^{1}, \mathbb{C}\right)$ с разложениями Фурье вида

$$
f(z)=\sum_{n \neq 0} f_{n} z^{n}, \quad z=e^{i \theta}
$$


и конечной соболевской нормой

$$
\|f\|_{1 / 2}^{2}=\sum_{n \neq 0}|n|\left|f_{n}\right|^{2}<\infty .
$$

Риманова метрика $g^{0}$ продолжается на $V^{\mathbb{C}}$ до эрмитовой метрики

$$
\langle\xi, \eta\rangle=\sum_{n \neq 0}|n| \xi_{n} \bar{\eta}_{n}
$$

Продолжим также форму $\omega$ и комплексную структуру $J^{0}$ комплексно линейно на $V^{\mathbb{C}}$. Тогда пространство $V^{\mathbb{C}}$ будет допускать разложение в прямую сумму подпространств

$$
V^{\mathbb{C}}=W_{+} \oplus W_{-},
$$

где $W_{ \pm}$есть собственное $(\mp i)$-подпространство линейного оператора $J^{0}: V^{\mathbb{C}} \rightarrow$ $V^{\mathbb{C}}$. Иными словами,

$$
W_{+}=\left\{\xi \in V^{\mathbb{C}}: \xi(z)=\sum_{n=1}^{\infty} \xi_{n} z^{n}\right\}, \quad W_{-}=\left\{\xi \in V^{\mathbb{C}}: \xi(z)=\sum_{n=-\infty}^{-1} \xi_{n} z^{n}\right\} .
$$

Подпространства $W_{ \pm}$изотропны относительно симплектической формы $\omega$, т. е. $\omega(\xi, \eta)=0$, если одновременно $\xi, \eta \in W_{+}$или $\xi, \eta \in W_{-}$. Разложение (34) является ортогональной прямой суммой относительно эрмитова скалярного произведения $\langle\cdot, \cdot\rangle$.

Обозначим через $\mathscr{D}$ пространство Дирихле, состоящее из гармонических функций в круге $h: \Delta \rightarrow \mathbb{R}$, нормированных условием $h(0)=0$ и имеющих конечную энергию

$$
E(h)=\frac{1}{2 \pi} \int_{\Delta}|\operatorname{grad} h(z)|^{2} d x d y=\frac{1}{2 \pi} \int_{\Delta}\left(\left|\frac{\partial h}{\partial x}\right|^{2}+\left|\frac{\partial h}{\partial y}\right|^{2}\right) d x d y<\infty .
$$

Хорошо известен следующий факт (см. [73]).

ПРЕДЛОЖЕНИЕ 8. Преобразование Пуассона

$$
\operatorname{Pf}(z)=\frac{1}{2 \pi} \int_{0}^{2 \pi} P(\zeta, z) f(\zeta) d \theta, \quad \zeta=e^{i \theta}
$$

где $P(\zeta, z)$ - ядро Пуассона в круге $\Delta$ :

$$
P(\zeta, z)=\frac{|\zeta|^{2}-|z|^{2}}{|\zeta-z|^{2}}
$$

устанавливает изометрический изоморфизм $P: V \rightarrow \mathscr{D}$ между соболевским пространством $V$ и пространством Дирихле $\mathscr{D}$, наделеннъм нормой $\|h\|_{\mathscr{D}}^{2}:=$ $E(h)$.

Пусть $f$ - сохраняющий ориентацию гомеоморфизм $S^{1} \rightarrow S^{1}$. Сопоставим ему оператор $T_{f}$, действующий по формуле

$$
\left(T_{f} \xi\right)(z)=\xi(f(z))-\frac{1}{2 \pi} \int_{0}^{2 \pi} \xi\left(f\left(e^{i \theta}\right)\right) d \theta, \quad z=e^{i \theta},
$$

на функциях $\xi \in V$. 
Tеорема 6 (теорема Нага-Сулливана). Onератор $T_{f}$ действует из пространства $V$ в себя тогда и только тогда, когда $f \in \operatorname{QS}\left(S^{1}\right)$. Если квазисимметричный гомеоморфизм $f$ продолжсается до $K$-квазиконформного гомеоморфизма круга $\Delta$, то операторная норма $T_{f}$ не превосходит $\sqrt{K+K^{-1}}$.

ДокАЗАТЕЛЬСтво. Достаточность. Пусть гомеоморфизм $f$ принадлежит $\operatorname{QS}\left(S^{1}\right)$ и продолжается до квазиконформного гомеоморфизма $w$ круга $\Delta$. Пусть $\xi$ - произвольный вектор из $V$ и $h=P \xi$ - его гармоническое продолжение внутрь $\Delta$. Тогда определено граничное значение функции $g:=h \circ w$, которое совпадает с $\xi \circ f$. Покажем, что $\xi \circ f \in V$, точнее, покажем, что энергия гармонического продолжения функции $\xi \circ f$ не превосходит

$$
E(P(\xi \circ f)) \leqslant 2\left(\frac{1+k^{2}}{1-k^{2}}\right) E(P f),
$$

где $k$ - константа квазиконформности $w$, равная норме $\|\mu\|_{\infty}$ дифференциала Бельтрами $\mu$ отображения $w$. Отсюда, в силу предложения 8 , будет следовать, что операторная норма $T_{f}$ не превосходит

$$
\left\|T_{f}\right\| \leqslant \sqrt{2} \sqrt{\frac{1+k^{2}}{1-k^{2}}}=\sqrt{K+\frac{1}{K}}, \quad \text { где } \quad K=\frac{1+k}{1-k} .
$$

Достаточно доказать неравенство (35) для отображения $g=h \circ w$, поскольку отсюда будет следовать его справедливость для $P(\xi \circ f)$. (Напомним, что минимум энергии среди всех гладких отображений с заданными граничными значениями достигается как раз на гармонических отображениях.) Полагая $w=u+i v$, получим оценку

$$
\left(\frac{\partial g}{\partial x}\right)^{2}+\left(\frac{\partial g}{\partial y}\right)^{2} \leqslant 2\left[\left(\frac{\partial h}{\partial u}\right)^{2}+\left(\frac{\partial h}{\partial v}\right)^{2}\right]\left(|\partial w|^{2}+|\bar{\partial} w|^{2}\right) \text { почти всюду в } \Delta .
$$

Квазиконформность отображения $w$ означает, что

$$
|\bar{\partial} w| \leqslant k|\partial w| \quad \text { почти всюду в } \Delta,
$$

поэтому

$$
\left(\frac{\partial g}{\partial x}\right)^{2}+\left(\frac{\partial g}{\partial y}\right)^{2} \leqslant 2\left[\left(\frac{\partial h}{\partial u}\right)^{2}+\left(\frac{\partial h}{\partial v}\right)^{2}\right]\left(\frac{1+k^{2}}{1-k^{2}}\right) \operatorname{Jac}(w),
$$

где $\operatorname{Jac}(w)=|\partial w|^{2}-|\bar{\partial} w|^{2}$. После замены переменных в интеграле для $E(g)$ получаем требуемое неравенство

$$
E(g) \leqslant 2\left(\frac{1+k^{2}}{1-k^{2}}\right) E(h) .
$$

Как известно, интеграл Дирихле обладает свойством конформной инвариантности. Приведенное доказательство показывает, по существу, что он обладает и свойством квазиинвариантности относительно квазиконформных отображений.

Необходимостъ. Пользуясь свойством конформной инвариантности интеграла Дирихле, можно свести доказываемое утверждение к случаю верхней полуплоскости $H$. При этом соболевское пространство $H_{0}^{1 / 2}\left(S^{1}, \mathbb{R}\right)$ заменится 
на соболевское пространство $H^{1 / 2}(\mathbb{R})$. Нам понадобится следующая формула Дугласа, выражающая энергию отображения $f \in H^{1 / 2}(\mathbb{R})$ через разностную производную функции $f$ (эта формула доказывается, например, в книге [10]):

$$
E(P f)=\|f\|_{1 / 2}^{2}=\frac{1}{4 \pi^{2}} \int_{\mathbb{R}} \int_{\mathbb{R}}\left[\frac{f(x)-f(y)}{x-y}\right]^{2} d x d y
$$

Пусть теперь $f$ есть сохраняющий ориентацию гомеоморфизм $f: \mathbb{R} \rightarrow \mathbb{R}$, для которого

$$
T_{f}^{-1}: H^{1 / 2}(\mathbb{R}) \rightarrow H^{1 / 2}(\mathbb{R})
$$

есть ограниченный оператор с нормой $M$. Выберем срезающую функцию $\chi_{0} \in$ $C_{0}^{\infty}(\mathbb{R}), 0 \leqslant \chi_{0} \leqslant 1$, такую, что $\chi_{0} \equiv 1$ на отрезке $[-1,1]$ и $\chi_{0} \equiv 0$ вне отрезка $[-2,2]$. Зафиксируем $x \in \mathbb{R}, t>0$ и введем обозначения

$$
I_{1}=[x-t, x], \quad I_{2}=[x, x+t] .
$$

Рассмотрим сдвиг функции $\chi_{0}$, задаваемый формулой

$$
\chi_{1}(s):=\chi_{0}(a s+b),
$$

где константы $a, b$ подбираются таким образом, чтобы $\chi_{1} \equiv 1$ на $I_{1}$ и $\chi_{1} \equiv 0$ на $[x+t, \infty)$.

По предположению $\chi_{1} \circ f^{-1} \in H^{1 / 2}(\mathbb{R})$ и ввиду ограниченности оператора $T_{f}^{-1}$ справедлива оценка

$$
\left\|T_{f}^{-1} \chi_{1}\right\|=\left\|\chi_{1} \circ f^{-1}\right\|_{1 / 2} \leqslant M\left\|\chi_{1}\right\|_{1 / 2}=M\left\|\chi_{0}\right\|_{1 / 2}
$$

(последнее равенство вытекает, например, из формулы Дугласа). Следовательно,

$$
\begin{aligned}
M^{2}\left\|\chi_{0}\right\|_{1 / 2}^{2} & \geqslant\left\|\chi_{1} \circ f^{-1}\right\|_{1 / 2}^{2}=\frac{1}{4 \pi^{2}} \int_{\mathbb{R}} \int_{\mathbb{R}}\left[\frac{\chi_{1} \circ f^{-1}(r)-\chi_{1} \circ f^{-1}(s)}{r-s}\right]^{2} d r d s \\
& \geqslant \int_{f(x-t)}^{f(x)} d s \int_{f(x+t)}^{\infty} d r \frac{1}{(r-s)^{2}}=\log \left[1+\frac{f(x)-f(x-t)}{f(x+t)-f(x)}\right] .
\end{aligned}
$$

Отсюда

$$
\frac{f(x)-f(x-t)}{f(x+t)-f(x)} \geqslant \frac{1}{\exp \left(M^{2}\left\|\chi_{0}\right\|_{1 / 2}^{2}\right)-1}
$$

для всех $x \in \mathbb{R}, t>0$.

Аналогично, выбирая сдвиг $\chi_{2}$ функции $\chi_{0}$ так, чтобы $\chi_{2} \equiv 1$ на $I_{2}$ и $\chi_{2} \equiv 0$ на $(-\infty, x-t]$, докажем противоположное неравенство

$$
\frac{f(x+t)-f(x)}{f(x)-f(x-t)} \leqslant \exp \left(M^{2}\left\|\chi_{0}\right\|_{1 / 2}^{2}\right)-1 .
$$

Из двух последних неравенств следует, что отображение $f$ удовлетворяет условию Бёрлинга-Альфорса (14), т. е. гомеоморфизм $f$ квазисимметричен. Теорема доказана. 
Tеорема 7 [73]. Действие операторов $T_{f}: V \rightarrow V c f \in \mathrm{QS}\left(S^{1}\right)$ на соболевском пространстве $V$ сохраняет симплектическую структуру $\omega$, m.e.

$$
\omega\left(T_{f} \xi, T_{f} \eta\right)=\omega(\xi, \eta) \quad \text { для любъх } \xi, \eta \in V .
$$

Более того, комплексно-линейное продолжение оператора $T_{f}$ на комплексифицированное пространство $V^{\mathbb{C}}$ сохраняет подпространства $W_{ \pm}$тогда и только тогда, когда $f \in \operatorname{Möb}\left(S^{1}\right)$, и в этом случае $T_{f}$ действует на $W_{ \pm}$как унитарный оператор.

Приведем идею доказательства этой теоремы. Докажем сначала первое утверждение теоремы. Оно, очевидно, выполняется для гладких гомеоморфизмов $f$. Действительно, на гладких векторах $\xi, \eta \in C_{0}^{\infty}\left(S^{1}, \mathbb{R}\right)$ утверждение сводится к формуле замены переменной, задаваемой отображением $f$. Аппроксимируя произвольные векторы $\xi, \eta \in V$ гладкими, покажем, что соотношение (38) выполняется и для них. Далее докажем справедливость соотношения (38) для произвольных гомеоморфизмов $f \in \mathrm{QS}\left(S^{1}\right)$, аппроксимируя их гладкими с помощью теоремы аппроксимации из [67].

Если действие $T_{f}$ на $V^{\mathbb{C}}$ сохраняет подпространство $W_{+}$, то $f$ продолжается до голоморфного отображения $F: \Delta \rightarrow \Delta$. Так как $f$ - гомеоморфизм, то отображение $F$ должно быть конформно, т. е. $F \in \operatorname{Möb}(\Delta)$. Поскольку оператор $T_{f}$ сохраняет симплектическую форму, то отсюда следует, что он сохраняет и эрмитову метрику на $W_{+}$, т. е. действует на $W_{+}$унитарными преобразованиями. Аналогичные рассуждения применимы и к $W_{-}$.

На самом деле, можно показать, что и сама форма $\omega$ на $V$ определена фактически единственным образом, точнее, имеет место следующая теорема.

Теорема 8 [73]. Допустим, что непрерывная билинейная форма $\widetilde{\omega}: V \times$ $V \rightarrow \mathbb{R}$ обладает следующим свойством инвариантности относительно дробно-линейных преобразований:

$$
\widetilde{\omega}\left(T_{f} \xi, T_{f} \eta\right)=\widetilde{\omega}(\xi, \eta) \quad \text { для любых } f \in \operatorname{Möb}\left(S^{1}\right), \xi, \eta \in V .
$$

Тогда $\widetilde{\omega}=\lambda \omega$ для некоторого $\lambda \in \mathbb{R}$. В частности, если такая форма не равна тождественно нулю, то она автоматически невырождена и инвариантна относительно всей группы квазисимметричных гомеоморфизмов $\mathrm{QS}\left(S^{1}\right)$.

Из теоремы Нага-Сулливана и теоремы 7 вытекает, что имеется вложение

$$
\mathscr{T}=\mathrm{QS}\left(S^{1}\right) / \operatorname{Möb}\left(S^{1}\right) \rightarrow \operatorname{Sp}(V) / \mathrm{U}\left(W_{+}\right),
$$

где $\operatorname{Sp}(V)$ обозначает симплектическую группу пространства $V$, состоящую из ограниченных линейных операторов на $V$, сохраняющих симплектическую форму $\omega$, а $\mathrm{U}\left(W_{+}\right)$- ее подгруппа, состоящая из унитарных операторов, т. е. операторов, комплексно-линейные продолжения которых на $V^{\mathbb{C}}$ сохраняют подпространство $W_{+}$(и, следовательно, $\left.W_{-}\right)$.

Опишем эти группы более подробно. В терминах разложения (34),

$$
V^{\mathbb{C}}=W_{+} \oplus W_{-},
$$

любой линейный оператор $A: V^{\mathbb{C}} \rightarrow V^{\mathbb{C}}$ может быть записан в блочной форме

$$
A=\left(\begin{array}{ll}
a & b \\
c & d
\end{array}\right)=\left(\begin{array}{ll}
a: W_{+} \rightarrow W_{+} & b: W_{-} \rightarrow W_{+} \\
c: W_{+} \rightarrow W_{-} & d: W_{-} \rightarrow W_{-}
\end{array}\right) .
$$


В частности, линейные операторы на $V^{\mathbb{C}}$, получаемые комплексно-линейным продолжением операторов $A: V \rightarrow V$, имеют блочные представления вида

$$
A=\left(\begin{array}{ll}
a & b \\
\bar{b} & \bar{a}
\end{array}\right)
$$

где мы отождествляем пространство $W_{-}$с комплексно-сопряженным пространством $\bar{W}_{+}$. Линейный оператор $A: V \rightarrow V$ принадлежит симплектической группе $\operatorname{Sp}(V)$, если он сохраняет симплектическую форму $\omega$. Это условие эквивалентно следующим соотношениям на блочные компоненты $A$ :

$$
A=\left(\begin{array}{ll}
a & b \\
\bar{b} & \bar{a}
\end{array}\right) \in \operatorname{Sp}(V) \quad \Longleftrightarrow \quad \bar{a}^{t} a-b^{t} \bar{b}=1, \bar{a}^{t} b=b^{t} \bar{a},
$$

где $a^{t}: W_{+}^{\prime} \rightarrow W_{+}^{\prime}$ и $b^{t}: W_{+}^{\prime} \rightarrow W_{-}^{\prime}$ обозначают транспонированные операторы, а пространство $W_{ \pm}^{\prime}$, двойственное к $W_{ \pm}$, отождествляется с пространством $W_{\mp}$ с помощью скалярного произведения $\langle\cdot, \cdot\rangle$ на $V^{\mathbb{C}}$, задаваемого комплексно-линейным продолжением на $V^{\mathbb{C}}$ римановой метрики $g^{0}$. Унитарная группа $\mathrm{U}\left(W_{+}\right)$ вкладывается в симплектическую группу $\operatorname{Sp}(V)$ в виде подгруппы блочно-диагональных матриц вида

$$
A=\left(\begin{array}{cc}
a & 0 \\
0 & \bar{a}
\end{array}\right)
$$

Вернемся к отображению (39). Пространство

$$
\operatorname{Sp}(V) / \mathrm{U}\left(W_{+}\right)
$$

в правой части (39) можно отождествить с пространством $\mathscr{J}(V)$ комплексных структур на пространстве $V^{\mathbb{C}}$, совместимых с симплектической формой $\omega$. Действительно, любая комплексная структура $J$ такого вида определяет разложение

$$
V^{\mathbb{C}}=W \oplus \bar{W}
$$

в прямую сумму собственных $(\mp i)$-подпространств оператора $J$, изотропных относительно $\omega$. Обратно, любое разложение вида (41) пространства $V^{\mathbb{C}}$ в прямую сумму подпространств, изотропных относительно $\omega$, определяет комплексную структуру $J$ на $V^{\mathbb{C}}$, равную $-i I$ на $W$ и $+i I$ на $\bar{W}$ и совместимую с $\omega$. Тем самым, группа $\operatorname{Sp}(V)$ действует транзитивно на пространстве $\mathscr{J}(V)$ комплексных структур $J$ на $V$, совместимых с $\omega$.

Чтобы получить однородное представление для пространства $\mathscr{J}(V)$, нужно профакторизовать группу $\operatorname{Sp}(V)$ по ее подгруппе, состоящей из преобразований, сохраняющих исходную комплексную структуру $J^{0}$ или, другими словами, сохраняющих подпространства $W_{ \pm}$. Указанная подгруппа состоит в точности из унитарных преобразований из группы $\mathrm{U}\left(W_{+}\right)$, откуда следует, что

$$
\mathscr{J}(V)=\operatorname{Sp}(V) / \mathrm{U}\left(W_{+}\right) .
$$

Пространство $\mathscr{J}(V)$ допускает интерпретацию в виде бесконечномерного зигелева диска. По определению, зигелев диск $\mathscr{D}$ состоит из ограниченных линейных операторов вида

$$
\begin{aligned}
\mathscr{D}=\{ & Z: W_{+} \rightarrow W_{-} \text {есть ограниченный линейный симметричный } \\
& \text { оператор, удовлетворяющий } \bar{Z} Z<I\} .
\end{aligned}
$$


Симметричность $Z$ означает, что $Z^{t}=Z$, а условие $\bar{Z} Z<I$ равносильно тому, что симметричный оператор $I-\bar{Z} Z$ положительно определен.

Для того чтобы отождествить пространство $\mathscr{J}(V)$ с зигелевым диском $\mathscr{D}$, рассмотрим действие группы $\operatorname{Sp}(V)$ на $\mathscr{D}$, задаваемое операторными дробно-линейными преобразованиями вида

$$
\mathrm{Sp}(V) \ni A=\left(\begin{array}{cc}
a & b \\
\bar{b} & \bar{a}
\end{array}\right): Z \mapsto(\bar{a} Z+\bar{b})(b Z+a)^{-1} .
$$

Нетрудно проверить, что сопоставление оператору $A \in \operatorname{Sp}(V)$ указанного дробно-линейного преобразования зигелева диска $\mathscr{D}$ задает взаимно однозначное отображение

$$
\mathscr{J}(V)=\operatorname{Sp}(V) / \mathrm{U}\left(W_{+}\right) \rightarrow \mathscr{D} .
$$

Зигелев диск $\mathscr{D}$ естественным образом вкладывается в грассманиан $\operatorname{Gr}_{b}\left(V^{\mathbb{C}}\right)$ гильбертова пространства $V^{\mathbb{C}}$, состоящий из замкнутых подпространств $W \subset$ $V^{\mathbb{C}}$, получаемых из $W_{+}$действием ограниченных линейных операторов. Указанное вложение задается отображением

$$
\mathscr{D} \ni Z \mapsto \text { график отображения } Z: W_{+} \rightarrow W_{-} .
$$

Грассманиан $\operatorname{Gr}_{b}\left(V^{\mathbb{C}}\right)$ является комплексным банаховым многообразием (см. [97]), а сквозное отображение

$$
\mathscr{T}=\operatorname{QS}\left(S^{1}\right) / \operatorname{Möb}\left(S^{1}\right) \rightarrow \operatorname{Sp}(V) / \mathrm{U}\left(W_{+}\right)=\mathscr{D} \rightarrow \operatorname{Gr}_{b}\left(V^{\mathbb{C}}\right)
$$

является эквивариантным голоморфным вложением комплексных банаховых многообразий (см. [73]).

Построенное вложение $\mathscr{T} \hookrightarrow \operatorname{Gr}_{b}\left(V^{\mathbb{C}}\right)$ порождает вложение пространства нормализованных диффеоморфизмов

$$
\mathscr{S}=\operatorname{Diff}_{+}\left(S^{1}\right) / \operatorname{Möb}\left(S^{1}\right) \subset \mathscr{T}
$$

в "регулярную часть" грассманиана $\operatorname{Gr}_{b}\left(V^{\mathbb{C}}\right)$, совпадающую с грассманианом Гильберта-Шмидта $\mathrm{Gr}_{\mathrm{HS}}(V)$, который определяется следующим образом.

ОПРедЕЛЕНИЕ 2. Грассманианом Гильберта-Шмидта $\operatorname{Gr}_{\mathrm{HS}}(V)$ называется множество, состоящее из замкнутых подпространств $W \subset V^{\mathbb{C}}$ таких, что ортогональная проекция $\pi_{+}: W \rightarrow W_{+}$является фредгольмовым оператором, а ортогональная проекция $\pi_{-}: W \rightarrow W_{-}$- оператором Гильберта-Шмидта.

Можно сказать, что $\operatorname{Gr}_{\mathrm{HS}}(V)$ состоит из замкнутых подпространств $W \subset$ $V^{\mathbb{C}}$, которые "мало" отличаются от подпространства $W_{+}$в том смысле, что проекция $\pi_{+}: W \rightarrow W_{+}$"почти" обратима, а проекция $\pi_{-}: W \rightarrow W_{-}$"мала".

Грассманиан $\operatorname{Gr}_{\mathrm{HS}}(V)$ является кэлеровым гильбертовым многообразием, имеющим в качестве локальной модели гильбертово пространство $\operatorname{HS}\left(W_{+}, W_{-}\right)$ операторов Гильберта-Шмидта.

Введем теперь симплектическую группу Гильберта-Шмидта $\operatorname{Sp}_{\mathrm{HS}}(V)$, которая состоит из преобразований

$$
A=\left(\begin{array}{ll}
a & b \\
\bar{b} & \bar{a}
\end{array}\right) \in \operatorname{Sp}(V),
$$


для которых $b$ является оператором Гильберта-Шмидта. Унитарная группа $\mathrm{U}\left(W_{+}\right)$содержится в $\operatorname{Sp}_{\mathrm{HS}}(V)$ в виде подгруппы блочно-диагональных матриц.

Построенное вложение $\mathscr{T} \hookrightarrow \mathscr{J}(V)$ индуцирует вложение

$$
\mathscr{S}=\operatorname{Diff}_{+}\left(S^{1}\right) / \operatorname{Möb}\left(S^{1}\right) \hookrightarrow \operatorname{Sp}_{\mathrm{HS}}(V) / \mathrm{U}\left(W_{+}\right) .
$$

Пространство

$$
\mathscr{J}_{\mathrm{HS}}(V):=\operatorname{Sp}_{\mathrm{HS}}(V) / \mathrm{U}\left(W_{+}\right)
$$

отождествляется, как и выше, с некоторым пространством комплексных структур на $V^{\mathbb{C}}$, совместимых с симплектической формой $\omega$. Будем называть комплексные структуры из $\mathscr{J}_{\mathrm{HS}}(V)$ комплексными структурами ГильбертаШмидта. Так же как выше, пространство $\mathscr{J}_{\mathrm{HS}}(V)$ допускает реализацию в виде зигелева диска Гилъберта-Шмидта, определяемого как

$$
\begin{aligned}
\mathscr{D}_{\mathrm{HS}}=\{ & \left\{: W_{+} \rightarrow W_{-}-\right.\text {симметричный оператор } \\
& \text { Гильберта-Шмидта с } \bar{Z} Z<I\} .
\end{aligned}
$$

Указанный зигелев диск $\mathscr{D}_{\mathrm{HS}}$ вкладывается, как и выше, в грассманиан Гильберта-Шмидта $\operatorname{Gr}_{\mathrm{HS}}(V)$ так, что сквозное отображение

$$
\mathscr{S}=\operatorname{Diff}_{+}\left(S^{1}\right) / \operatorname{Möb}\left(S^{1}\right) \hookrightarrow \mathscr{J}_{\mathrm{HS}}(V)=\operatorname{Sp}_{\mathrm{HS}}(V) / \mathrm{U}\left(W_{+}\right)=\mathscr{D}_{\mathrm{HS}} \hookrightarrow \operatorname{Gr}_{\mathrm{HS}}(V)
$$

является эквивариантным голоморфным вложением комплексного пространства $\Phi$ реше $\mathscr{S}$ в комплексное гильбертово многообразие $\operatorname{Gr}_{\mathrm{HS}}(V)$ (см. [72]).

\section{6. Квантование пространства нормализованных диффеоморфиз-} мов. Геометрическому квантованию классических систем в смысле Дирака посвящен целый ряд книг, например, монография Вудхауса [126]. Начнем с описания геометрического квантования конечномерных (механических) систем.

Конечномерная классическая система задается парой $(M, \mathscr{A})$, состоящей из фазового пространства $M$ и алгебры наблюдаемых $\mathscr{A}$.

Фазовое пространство $M$ есть гладкое симплектическое многообразие четной размерности $2 n$ с симплектической формой $\omega$. Локально оно изоморфно стандартной модели $M_{0}:=\left(\mathbb{R}^{2 n}, \omega_{0}\right)$, где $\omega_{0}$ - стандартная симплектическая форма на $\mathbb{R}^{2 n}$, задаваемая в канонических координатах $\left(p_{i}, q_{i}\right), i=1, \ldots, n$, на $\mathbb{R}^{2 n}$ формулой

$$
\omega_{0}=\sum_{i=1}^{n} d p_{i} \wedge d q_{i} .
$$

Алгебра наблюдаемых $\mathscr{A}$ есть произвольная подалгебра Ли в алгебре Ли $C^{\infty}(M, \mathbb{R})$ гладких вещественнозначных функций на фазовом пространстве $M$ относительно скобки Пуассона, определяемой симплектической формой $\omega$. В частности, $\mathscr{A}$ может совпадать со всей алгеброй Пуассона $C^{\infty}(M, \mathbb{R})$. В случае стандартной модели $M_{0}=\left(\mathbb{R}^{2 n}, \omega_{0}\right)$ в качестве алгебры наблюдаемых можно взять алгебру Гейзенберга heis $\left(\mathbb{R}^{2 n}\right)$, которая порождается координатными функциями $p_{i}, q_{i}, i=1, \ldots, n$, и 1, удовлетворяющими коммутационным соотношениям:

$$
\begin{aligned}
& \left\{p_{i}, p_{j}\right\}=\left\{q_{i}, q_{j}\right\}=0, \\
& \left\{p_{i}, q_{j}\right\}=\delta_{i j} \quad \text { при } i, j=1, \ldots, n .
\end{aligned}
$$


Алгебры наблюдаемых возникают обычно следующим образом. Пусть Г есть некоторая группа Ли, действующая на односвязном фазовом многообразии $M$ симплектическими преобразованиями. Тогда ее алгебру Ли Lie(Г) можно рассматривать как подалгебру алгебры Ли гамильтоновых векторных полей $X_{f}$ на $M$, порождаемых гладкими функциями $f \in C^{\infty}(M, \mathbb{R})$. В этом случае за алгебру наблюдаемых $\operatorname{ham}(\Gamma)$, отвечающую группе $\Gamma$, можно взять алгебру Ли, состоящую из функций $f$, для которых $X_{f} \in \operatorname{Lie}(\Gamma)$, и наделенную скобкой Пуассона в качестве скобки Ли.

Пусть $(M, \mathscr{A})$ есть некоторая классическая система. Квантованием этой системы называется неприводимое линейное представление

$$
r: \mathscr{A} \rightarrow \mathrm{End}^{*} H
$$

наблюдаемых из $\mathscr{A}$ самосопряженными линейными операторами, действующими в комплексном гильбертовом пространстве $H$, называемом пространством квантования. При этом требуется, чтобы

$$
r(\{f, g\})=\frac{1}{i}[r(f), r(g)]=\frac{1}{i}(r(f) r(g)-r(g) r(f))
$$

для любых $f, g \in \mathscr{A}$ и $r(1)=I$.

Операторы квантования $r(f)$, возникающие в конкретных примерах, оказываются, как правило, неограниченными, поэтому необходимо требовать, чтобы все они были определены на общей области определения, плотной в $H$.

Часто бывает удобнее иметь дело с комплексифицированными алгебрами наблюдаемых $\mathscr{A}^{\mathbb{C}}$ или, более общим образом, с инволютивными комплексными алгебрами наблюдаемых $\mathscr{A}_{\mathbb{C}}$, наделенными инволюцией. В этом случае квантование алгебры наблюдаемых $\mathscr{A}_{\mathbb{C}}$ будет задаваться неприводимым линейным представлением $r: \mathscr{A}_{\mathbb{C}} \rightarrow$ End $H$ замкнутыми линейными операторами на $H$, удовлетворяющим помимо условия (42) и нормировки $r(1)=I$ еще и правилу сопряжения: инволюция в $\mathscr{A}_{\mathbb{C}}$ переходит под действием $r$ в эрмитово сопряжение.

Мы будем применять приведенное определение квантования к бесконечномерным классическим системам, в которых как фазовые пространства, так и алгебры наблюдаемых являются бесконечномерными. Для бесконечномерных алгебр Ли $\mathscr{A}$ более естественно искать не обычные, а проективные представления. Если нам удастся найти такое представление для заданной алгебры наблюдаемых $\mathscr{A}$, то это будет означать, что мы построили квантование не исходной системы $(M, \mathscr{A})$, а ее расширения $(M, \tilde{\mathscr{A}})$, где $\tilde{\mathscr{A}}$ - подходящее центральное расширение алгебры $\mathscr{A}$, которое определяется коциклом проективного представления.

В случае пространства нормализованных диффеоморфизмов

$$
\mathscr{S}=\operatorname{Diff}_{+}\left(S^{1}\right) / \operatorname{Möb}\left(S^{1}\right)
$$

роль бесконечномерной классической системы будет играть пара

$$
\left(\mathscr{S}, \operatorname{Vect}\left(S^{1}\right)\right)
$$

где $\mathscr{S}$ - фазовое пространство системы, а $\operatorname{Vect}\left(S^{1}\right)$ - алгебра наблюдаемых, являющаяся алгеброй Ли группы Diff $+\left(S^{1}\right)$. Эта алгебра совпадает с алгеброй Ли гладких векторных полей на $S^{1}$. 
Квантование указанной системы было построено в книге [97], где была также рассмотрена связь пространства диффеоморфизмов $\mathscr{S}$ с теорией гладких струн. Ключевую роль в этом квантовании играет теорема Шейла-Березина, доказанная в оригинальной статье Шейла [100] и книге Березина [14].

Мы построим квантование указанной системы, предварительно расширив ее до системы, ассоциированной с соболевским пространством $V$. Для этого воспользуемся построенным выше вложением

$$
\mathscr{S} \hookrightarrow \mathscr{J}_{\mathrm{HS}}(V)=\operatorname{Sp}_{\mathrm{HS}}(V) / \mathrm{U}\left(W_{+}\right) .
$$

При таком вложении группа $\operatorname{Diff}_{+}\left(S^{1}\right)$ реализуется в виде подгруппы симплектической группы Гильберта-Шмидта $\operatorname{Sp}_{\mathrm{HS}}(V)$. В качестве расширенной классической системы берется пара

$$
\left(\mathscr{J}_{\mathrm{HS}}(V)=\mathscr{D}_{\mathrm{HS}}, \operatorname{sp}_{\mathrm{HS}}(V)\right),
$$

где $\operatorname{sp}_{\mathrm{HS}}(V)$ - алгебра Ли симплектической группы Гильберта-Шмидта $\operatorname{Sp}_{\mathrm{HS}}(V)$.

Приступая к квантованию расширенной системы $\left(\mathscr{J}_{\mathrm{HS}}(V), \operatorname{sp}_{\mathrm{HS}}(V)\right)$, необходимо прежде всего указать пространство квантования $H$, в котором реализуется представление алгебры наблюдаемых $\operatorname{sp}_{\mathrm{HS}}(V)$. Роль этого пространства в рассматриваемом случае будет играть фоковское пространство, ассоциированное с соболевским пространством $V$. Фоковское пространство и связанное с ним представление Гейзенберга подробно изучены в целом ряде книг, например, в монографии Березина [14] и лекциях Каца [50].

Для того чтобы определить указанное пространство, фиксируем некоторую комплексную структуру $J \in \mathscr{J}(V)$, совместимую с симплектической формой $\omega$. Эта структура порождает разложение комплексифицированного пространства $V^{\mathbb{C}}$ в прямую сумму

$$
V^{\mathbb{C}}=W \oplus \bar{W}
$$

собственных $(\mp i)$-подпространств оператора $J$. Указанное разложение ортогонально относительно эрмитова скалярного произведения на $V^{\mathbb{C}}$, порождаемого $J$ и $\omega$ :

$$
\langle z, w\rangle_{J}:=\omega(z, J w)
$$

Фоковское пространство $F\left(V^{\mathbb{C}}, J\right)$ является пополнением алгебры симметричных полиномов от переменных $z \in W$ по норме, порожденной скалярным произведением $\langle\cdot, \cdot\rangle_{J}$.

Более подробно, обозначим через $\mathfrak{S}(W)$ алгебру симметричных полиномов от переменных $z \in W$ и введем на ней скалярное произведение, порождаемое скалярным произведением $\langle\cdot, \cdot\rangle_{J}$. На мономах одинаковой степени оно задается формулой

$$
\left\langle z_{1} \otimes \cdots \otimes z_{n}, z_{1}^{\prime} \otimes \cdots \otimes z_{n}^{\prime}\right\rangle_{J}:=\sum_{\left\{i_{1}, \ldots, i_{n}\right\}}\left\langle z_{1}, z_{i_{1}}^{\prime}\right\rangle_{J} \cdots\left\langle z_{n}, z_{i_{n}}^{\prime}\right\rangle_{J},
$$

где суммирование ведется по всем перестановкам $\left\{i_{1}, \ldots, i_{n}\right\}$ множества $\{1, \ldots, n\}$ (скалярное произведение мономов разных степеней полагается равным нулю). Скалярное произведение на мономах продолжается затем по линейности на всю алгебру $\mathfrak{S}(W)$. 
Фоковское пространство

$$
F_{J}=F\left(V^{\mathbb{C}}, J\right)
$$

есть замыкание алгебры $\mathfrak{S}(W)$ по норме $\langle\cdot, \cdot\rangle_{J}$.

Если $\left\{w_{n}\right\}_{n=1}^{\infty}$ есть ортонормированный базис пространства $W$, то в качестве ортонормированного базиса фоковского пространства $F_{J}$ можно взять мономы вида

$$
P_{K}(z)=\frac{1}{\sqrt{k !}}\left\langle z, w_{1}\right\rangle_{J}^{k_{1}} \cdots\left\langle z, w_{n}\right\rangle_{J}^{k_{n}}, \quad z \in W,
$$

где $K=\left(k_{1}, \ldots, k_{n}, 0, \ldots\right)$ - финитный набор натуральных чисел $k_{i} \in \mathbb{N}$ и $k !=$ $k_{1} ! \cdots k_{n}$ !. Тем самым, фоковское пространство разлагается в прямую сумму

$$
F_{J}=\bigoplus_{k=0}^{\infty} \mathfrak{S}_{k}(W)
$$

где $\mathfrak{S}_{k}(W)$ есть подпространство полиномов степени $k$ в $\mathfrak{S}(W)$.

Алгеброй Гейзенберга heis $(V)$ гильбертова пространства $V$ называется центральное расширение абелевой алгебры Ли $V$, порождаемой координатными функциями. Другими словами, как векторное пространство, эта алгебра совпадает с

$$
\operatorname{heis}(V)=V \oplus \mathbb{R}
$$

и наделяется скобкой Ли вида

$$
[(x, s),(y, t)]:=(0, \omega(x, y)), \quad x, y \in V, \quad s, t \in \mathbb{R} .
$$

Построим неприводимое представление алгебры Гейзенберга heis $(V)$ в фоковском пространстве $F_{J}$. Заметим, прежде всего, что элементы алгебры $\mathfrak{S}(W)$ можно рассматривать как голоморфные функции на пространстве $\bar{W}$, отождествляя $z \in W$ с голоморфной функцией

$$
\bar{W} \ni \bar{w} \mapsto\langle z, w\rangle_{J} \quad \text { на } \bar{W} .
$$

Соответственно, пространство $F_{J}$ можно рассматривать как пространство функций, голоморфных на $\bar{W}$.

С учетом этого отождествления, представление Гейзенберга $r_{J}$ алгебры Гейзенберга heis $(V)$ в фоковском пространстве $F_{J}$ будет задаваться формулой

$$
V \ni v \mapsto r_{J}(v) f(\bar{w})=\partial_{v} f(\bar{w})+\langle v, w\rangle_{J} f(\bar{w}),
$$

где $\partial_{v}$ есть оператор дифференцирования в направлении вектора $v$. Продолжая $r_{J}$ на комплексифицированную алгебру heis ${ }^{\mathbb{C}}(V)$ той же формулой $(44)$, получим, что

$$
\begin{array}{ll}
r_{J}(\bar{z}) f(\bar{w})=\partial_{\bar{z}} f(\bar{w}) & \text { при } \bar{z} \in \bar{W}, \\
r_{J}(z) f(\bar{w})=\langle z, w\rangle_{J} f(\bar{w}) & \text { при } z \in W .
\end{array}
$$

Представление Гейзенберга удобно описывать в терминах операторов рождения и уничтожения на пространстве $F_{J}$, которые задаются формулами

$$
a_{J}^{*}(v)=\frac{r_{J}(v)+i r_{J}(J v)}{2}, \quad a_{J}(v)=\frac{r_{J}(v)-i r_{J}(J v)}{2},
$$


где $v \in V^{\mathbb{C}}$. Отсюда следует, что

$$
\begin{array}{ll}
a_{J}^{*}(z) f(\bar{w})=\langle z, w\rangle_{J} f(\bar{w}) & \text { при } z \in W, \\
a_{J}(\bar{z}) f(\bar{w})=\partial_{\bar{z}} f(\bar{w}) & \text { при } \bar{z} \in \bar{W} .
\end{array}
$$

Выбирая ортонормированный базис $\left\{w_{n}\right\}_{n=1}^{\infty}$ в пространстве $W$, введем операторы

$$
a_{n}^{*}:=a^{*}\left(w_{n}\right), \quad a_{n}:=a\left(\bar{w}_{n}\right) \quad \text { при } n=1,2, \ldots .
$$

Эти операторы удовлетворяют коммутационным соотношениям вида

$$
\left[a_{m}, a_{n}\right]=\left[a_{m}^{*}, a_{n}^{*}\right]=0, \quad\left[a_{m}^{*}, a_{n}\right]=\delta_{m n} I \quad \text { при } m, n \in \mathbb{N} .
$$

Вектор $f_{J} \in F_{J} \backslash\{0\}$ называется вакуумом, если он аннулируется всеми операторами уничтожения, т. е.

$$
a_{n} f_{J}=0 \quad \text { при всех } n=1,2, \ldots
$$

Такой вектор определяется представлением $r_{J}$ однозначно с точностью до мультипликативной константы. В случае исходного фоковского пространства $F_{0}=$ $F\left(V, J^{0}\right)$ в качестве вакуума берется $f_{0} \equiv 1$.

Действуя на вакуум $f_{J}$ операторами рождения $a_{n}^{*}$, мы получим множество векторов в $F_{J}$ вида $\left(a_{1}^{*}\right)^{k_{1}} \cdots\left(a_{n}^{*}\right)^{k_{n}} f_{J}$, замкнутая линейная оболочка которого совпадает со всем пространством $F_{J}$, откуда вытекает неприводимость представления $r_{J}$. Заметим, что мономы $P_{K}(z)$, задаваемые формулой $(43)$, которые были выбраны нами в качестве ортонормированного базиса пространства $F_{J}$, построены именно таким способом.

Мы хотим построить унитарный оператор $U_{J}: F_{0} \rightarrow F_{J}$, сплетающий представления Гейзенберга $r_{0}$ в пространстве $F_{0}$ и $r_{J}$ в пространстве $F_{J}$.

Теорема 9 (теорема Шейла-Березина [100], [14]). Пусть комплексная структура $J \in \mathscr{J}(V)$ получается из комплексной структуры $J^{0}$ действием элемента $A \in \mathrm{Sp}(V)$. Тогда представления $r_{0}$ в пространстве $F_{0}$ и $r_{J}$ в пространстве $F_{J}$ унитарно эквивалентны тогда и только тогда, когда $A \in \operatorname{Sp}_{\mathrm{HS}}(V)$. Другими словами, при выполнении последнего условия существует унитарный сплетающий оператор $U_{J}: F_{0} \rightarrow F_{J}$ такой, что

$$
r_{J}=U_{J} \circ r_{0} \circ U_{J}^{-1} \text {. }
$$

Приведем идею доказательства этой теоремы. Для того чтобы построить сплетающий оператор $U_{J}$, достаточно, согласно рассуждению, приведенному выше, построить вакуум в пространстве $F_{J}$. Указанный вакуум можно искать, раскладывая его по базису пространства $F_{0}$, образованному векторами $(1 / \sqrt{k !})\left(a_{1}^{*}\right)^{k_{1}} \cdots\left(a_{n}^{*}\right)^{k_{n}} f_{0}$, и подставляя полученный ряд в соотношения $(46)$. Искомый вакуум $f_{J}$ будет задаваться формулой

$$
f_{J}=c e^{-a_{J}^{*}\left(a^{-1} b\right) a_{J}^{*} / 2} f_{0},
$$

если $A=\left(\begin{array}{ll}a & b \\ \bar{b} & \bar{a}\end{array}\right)$. Коэффициент $c$ в этой формуле равен $c=\theta\left(\operatorname{det} a \bar{a}^{t}\right)^{-1 / 4}$, где $\theta$ - комплексное число, по модулю равное 1. Заметим, что из описания группы $\operatorname{Sp}(V)$, даваемого соотношением (40), следует, что оператор а обратим. 
Более того, вектор $f_{J}$, задаваемый формулой (47), принадлежит пространству $F_{J}$ тогда и только тогда, когда $a^{-1} b$ есть оператор Гильберта-Шмидта $\Longleftrightarrow b$ есть оператор Гильберта-Шмидта, т. е. $A \in \mathrm{Sp}_{\mathrm{HS}}(V)$. В этом случае оператор $a \bar{a}^{t}=1+b \bar{b}^{t}$ имеет вид " $1+$ ядерный" и потому его детерминант имеет смысл. Неопределенный коэффициент $\theta$ возникает из-за того, что вакуум $f_{J}$ определяется только с точностью до мультипликативной константы, по модулю равной 1. Имея формулу (47) для вакуума $f_{J}$, можно найти и явное представление для сплетающего оператора $U_{J}$, которое выписано в [14]. Этот оператор, так же как и вакуум, определяется однозначно с точностью до мультипликативной константы, по модулю равной 1.

Объединим все фоковские пространства $F_{J}$ с $J \in \mathscr{J}_{\mathrm{HS}}(V)$ в единое фоковское расслоение

$$
\mathscr{F}=\bigcup_{J \in \mathscr{J}_{\mathrm{HS}}(V)} F_{J} \rightarrow \mathscr{J}_{\mathrm{HS}}(V)=\mathscr{D}_{\mathrm{HS}} .
$$

ПрЕДЛОЖЕНИЕ 9. Фоковское расслоение $\mathscr{F} \rightarrow \mathscr{J}_{\mathrm{HS}}(V)$ является эрмитовым голоморфным гильбертовым расслоением над зигелевым диском $\mathscr{D}_{\mathrm{HS}}$. На нем имеется унитарное проективное действие группы $\operatorname{Sp}_{\mathrm{HS}}(V)$, накрывающее естественное действие этой группы на $\mathscr{J}_{\mathrm{HS}}(V)=\mathscr{D}_{\mathrm{HS}}$.

Голоморфность фоковского расслоения устанавливается так же, как голоморфность детерминантного расслоения над грассманианом Гильберта-Шмидта $\operatorname{Gr}_{\mathrm{HS}}(V)$ (см. [97]). Поскольку зигелев диск $\mathscr{D}_{\mathrm{HS}}$ является стягиваемым (и даже выпуклым) множеством, то это расслоение тривиально. Более того, действие группы $\operatorname{Sp}_{\mathrm{HS}}(V)$, определяемое теоремой Шейла-Березина, задает его явную тривиализацию.

Инфинитезимальным вариантом действия симплектической группы Гильберта-Шмидта $\operatorname{Sp}_{\mathrm{HS}}(V)$ на фоковском расслоении является проективное представление ее алгебры Ли $\operatorname{sp}_{\mathrm{HS}}(V)$ в слое $F_{0}=F\left(V^{\mathbb{C}}, J^{0}\right)$ фоковского расслоения над точкой $J^{0}$. Это представление симплектической алгебры Гильберта-Шмидта построено в статье Сигала [96] (см. также [90], [97]).

Симплектическая алгебра Ли $\operatorname{sp}_{\mathrm{HS}}(V)$ состоит из ограниченных линейных операторов $A$, действующих в пространстве $V^{\mathbb{C}}$ и имеющих блочные представления вида

$$
A=\left(\begin{array}{cc}
\alpha & \beta \\
\bar{\beta} & \bar{\alpha}
\end{array}\right)
$$

где $\alpha$ - ограниченный косоэрмитов оператор, $\beta$ - симметричный оператор Гильберта-Шмидта. Комплексифицированная алгебра Ли $\operatorname{sp}_{\mathrm{HS}}(V)^{\mathbb{C}}$ состоит из операторов вида

$$
A=\left(\begin{array}{cc}
\alpha & \beta \\
\bar{\gamma} & -\alpha^{t}
\end{array}\right)
$$

где $\alpha$ - ограниченный оператор, а $\beta$ и $\bar{\gamma}$ являются симметричными операторами Гильберта-Шмидта.

Проективное представление комплексифицированной симплектической алгебры $\operatorname{sp}_{\mathrm{HS}}(V)^{\mathbb{C}}$ в пространстве $F_{0}$ задается формулой

$$
\operatorname{sp}_{\mathrm{HS}}(V)^{\mathbb{C}} \ni A=\left(\begin{array}{cc}
\alpha & \beta \\
\bar{\gamma} & -\alpha^{t}
\end{array}\right) \mapsto \rho(A)=D_{\alpha}+\frac{1}{2} M_{\beta}+\frac{1}{2} M_{\gamma}^{*} .
$$


Здесь $D_{\alpha}$ - оператор дифференцирования, порождаемый оператором $\alpha: W_{+} \rightarrow$ $W_{+}$и определяемый посредством

$$
D_{\alpha} f(\bar{w})=\left\langle\alpha w, \partial_{w}\right\rangle f(\bar{w}) .
$$

Оператор $M_{\beta}$, порождаемый оператором $\beta: W_{-}=\bar{W}_{+} \rightarrow W_{+}$, имеет вид

$$
M_{\beta} f(\bar{w})=\langle\bar{\beta} w, w\rangle f(\bar{w}),
$$

а оператор $M_{\gamma}^{*}$, сопряженный к $M_{\gamma}$, действует по формуле

$$
M_{\gamma}^{*} f(\bar{w})=\left\langle\gamma \partial_{w}, \partial_{w}\right\rangle f(\bar{w}) .
$$

Теорема 10 (Сигал [96]). Формула (48) задает унитарное проективное представление симплектической алгебры Ли $\mathrm{sp}_{\mathrm{HS}}(V)^{\mathbb{C}}$ в фоковском пространстве $F_{0}$ с коииклом

$$
\left[\rho\left(A_{1}\right), \rho\left(A_{2}\right)\right]-\rho\left(\left[A_{1}, A_{2}\right]\right)=\frac{1}{2} \operatorname{tr}\left(\bar{\gamma}_{2} \beta_{1}-\bar{\gamma}_{1} \beta_{2}\right) I .
$$

Это представление сплетается с представлением Гейзенберга $r_{0}$ алгебры Гейзенберга heis $(V)$ в пространстве $F_{0}$.

Тот факт, что построенное представление симплектической алгебры Гильберта-Шмидта сплетается с представлением $r_{0}$, вытекает из того, что это представление является инфинитезимальной версией проективного действия симплектической группы Гильберта-Шмидта на фоковском расслоении, сплетающего различные представления алгебры Гейзенберга.

Проективное представление алгебры Ли $\operatorname{sp}_{\mathrm{HS}}(V)$ определяет квантование расширенной системы

$$
\left(\mathscr{J}_{\mathrm{HS}}(V)=\mathscr{D}_{\mathrm{HS}}, \widetilde{\mathrm{sp}_{\mathrm{HS}}(V)}\right),
$$

где $\widetilde{\operatorname{sp}_{\mathrm{HS}}(V)}$ есть центральное расширение алгебры Ли $\operatorname{sp}_{\mathrm{HS}}(V)$, задаваемое коциклом (49).

Одновременно мы построили квантование еще одной классической системы, тесно связанной с теорией струн. А именно системы $(V, \mathscr{A})$, фазовое пространство которой совпадает с соболевским пространством $V=H_{0}^{1 / 2}\left(S^{1}, \mathbb{R}\right)$, а алгебра наблюдаемых $\mathscr{A}$ есть полупрямая сумма $\mathscr{A}=\operatorname{heis}(V) \rtimes \operatorname{sp}_{\mathrm{HS}}(V)$. Указанную алгебру наблюдаемых можно рассматривать как бесконечномерный аналог алгебры Пуанкаре пространства Минковского. Напомним, что алгебра Пуанкаре есть полупрямая сумма алгебры трансляций и алгебры гиперболических поворотов пространства Минковского. В случае соболевского пространства $V$ роль алгебры трансляций играет алгебра Гейзенберга, а роль алгебры поворотов симплектическая алгебра Ли $\operatorname{sp}_{\mathrm{HS}}(V)$. В случае пространства Минковского преобразования из алгебры сдвигов линейно зависят от координат, а преобразования из алгебры поворотов зависят от них квадратично. Эта закономерность сохраняется и в бесконечномерном случае - представление Гейзенберга линейно по переменным $\bar{w}$ и $\partial_{\bar{w}}$, а представление алгебры Ли $\operatorname{sp}_{\mathrm{HS}}(V)$ по ним квадратично.

Сужение конструкции фоковского расслоения $\mathscr{F} \rightarrow \mathscr{D}_{\mathrm{HS}}$, приведенной выше, на подмногообразие

$$
\mathscr{S}=\operatorname{Diff}_{+}\left(S^{1}\right) / \operatorname{Möb}\left(S^{1}\right) \subset \mathscr{J}_{\mathrm{HS}}(V)=\mathscr{D}_{\mathrm{HS}}
$$


дает фоковское расслоение

$$
\mathscr{F}_{\mathscr{S}}:=\bigcup_{J \in \mathscr{S}} F_{J} \rightarrow \mathscr{S}
$$

над пространством $\mathscr{S}$.

ПрЕДЛОЖЕНИЕ 10. Фоковское расслоение $\mathscr{F}_{\mathscr{S}} \rightarrow \mathscr{S}$ является эрмитовым голоморфным гильбертовым расслоением над пространством $\mathscr{S}$. На этом расслоении имеется унитарное проективное действие группы диффеоморфизмов $\operatorname{Diff}_{+}\left(S^{1}\right)$, накрывающее естественное действие этой группы на $\mathscr{S}$.

Фоковское расслоение $\mathscr{F} \mathscr{S} \rightarrow \mathscr{S}$ тривиально, поскольку пространство $\mathscr{S}$ стягиваемо. Действие группы $\operatorname{Diff}_{+}\left(S^{1}\right)$ на расслоении $\mathscr{F} \mathscr{S}$ задается ограничением $\operatorname{Sp}_{\mathrm{HS}}(V)$-действия на фоковском расслоении $\mathscr{F} \rightarrow \mathscr{D}_{\mathrm{HS}}$, построенного выше. Однако это действие можно построить и непосредственно, как в статье [35].

Инфинитезимальным вариантом действия группы Diff $+\left(S^{1}\right)$ на фоковском расслоении $\mathscr{F}_{\mathscr{S}}$ является проективное представление алгебры Ли $\operatorname{Vect}\left(S^{1}\right)$ этой группы в фоковском пространстве $F_{0}$. Это представление, называемое представлением Вирасоро, хорошо известно и представлено в целом ряде статей и книг (например, в книгах [50], [90]).

Его конструкцию удобно описать в терминах введенных выше операторов рождения и уничтожения $a_{n}^{*}, a_{n}$ на пространстве $F_{0}$. Дополним это определение, полагая $a_{0}=\lambda I, \lambda \in \mathbb{R}$, и $a_{-n}:=n a_{n}^{*}, n \in \mathbb{N}$, так что для полученных операторов будут выполняться следующие коммутационные соотношения:

$$
\left[a_{m}, a_{n}\right]=m \delta_{m,-n} I \quad \text { при } m, n \in \mathbb{Z} .
$$

Представление Вирасоро алгебры Ли $\operatorname{Vect}^{\mathbb{C}}\left(S^{1}\right)$ порождается операторами Bupacopo $L_{n}$, являющимися образами базисных элементов $e_{n}$ алгебры

$$
\operatorname{Vect}^{\mathbb{C}}\left(S^{1}\right) \text {. }
$$

Эти операторы задаются формулой

$$
L_{n}=\frac{1}{2} \sum_{i=-\infty}^{\infty}: a_{-i} a_{i+n}:, \quad n \in \mathbb{Z},
$$

где знак : : означает нормальное упорядочение, определяемое правилом

$$
: a_{i} a_{j}:= \begin{cases}a_{i} a_{j} & \text { при } i \leqslant j \\ a_{j} a_{i} & \text { при } i>j .\end{cases}
$$

В частности, оператор энергии $L_{0}$ имеет вид $L_{0}=\lambda^{2} / 2+\sum_{i>0} a_{-i} a_{i}$. Из-за нормального упорядочения, при применении оператора $L_{n}$ к любому полиному $P$ из алгебры $\mathfrak{S}\left(W_{+}\right)$только конечное число членов в бесконечном ряде, задающем $L_{n} P$, будет отлично от нуля, т. е. действие операторов $L_{n}$ корректно определено на алгебре $\mathfrak{S}\left(W_{+}\right)$и продолжается на все фоковское пространство $F_{0}=\widehat{\mathfrak{S}\left(W_{+}\right)}$по замыканию. 
Операторы $L_{n}$, удовлетворяющие коммутационным соотношениям

$$
\left[L_{m}, L_{n}\right]=(m-n) L_{m+n}+\delta_{m,-n} \frac{m^{3}-m}{12},
$$

порождают унитарное проективное представление алгебры Ли $\operatorname{Vect}\left(S^{1}\right)$ в фоковском пространстве $F_{0}$ (см. [50]).

Построенное проективное представление алгебры Ли $\operatorname{Vect}\left(S^{1}\right)$ в фоковском пространстве $F_{0}$ задает квантование системы $(\mathscr{S}$, vir), где vir есть центральное расширение алгебры Ли $\operatorname{Vect}\left(S^{1}\right)$. Это расширение называется алгеброй Вирасоро и определяется коциклом представления (50). Заметим, что центральное расширение $\operatorname{Vect}\left(S^{1}\right)$ определяется по существу единственным образом (см. [97]).

3.7. Квантование универсального пространства Тейхмюллера. Настоящий пункт посвящен построению геометрического квантования универсального пространства Тейхмюллера. В его основе лежит схема квантования по Конну, которая в отличие от описанной ранее схемы квантования по Дираку допускает распространение на случай негладких наблюдаемых. Геометрическое квантование по Конну представлено в его книге [24] (см. также [37]). Основываясь на конновском подходе, мы строим квантование универсального пространства Тейхмюллера, предложенное ранее в статьях [98], [99].

В основе квантования расширенной системы $\left(\mathscr{J}_{\mathrm{HS}}(V), \operatorname{sp}_{\mathrm{HS}}(V)\right)$, а следовательно, и пространства нормализованных диффеоморфизмов $\left(\mathscr{S}, \operatorname{Vect}\left(S^{1}\right)\right)$ лежал тот факт, что естественное действие группы $\operatorname{Sp}_{\mathrm{HS}}(V)$ на пространстве $\mathscr{J}_{\mathrm{HS}}(V)=\operatorname{Sp}_{\mathrm{HS}}(V) / \mathrm{U}\left(W_{+}\right)$удалось поднять с помощью теоремы Шейла-Березина до проективного действия этой группы на фоковском расслоении

$$
\mathscr{F}=\bigcup_{J \in \mathscr{J}_{\mathrm{HS}}(V)} F_{J} \rightarrow \mathscr{J}_{\mathrm{HS}}(V) .
$$

Однако этот метод не применим для всего универсального пространства Тейхмюллера $\mathscr{T}$. Хотя у нас по-прежнему имеются вложение $\mathscr{T} \hookrightarrow \mathscr{J}=\mathrm{Sp}(V) /$ $\mathrm{U}\left(W_{+}\right)$пространства $\mathscr{T}$ в пространство $\mathscr{J}$ комплексных структур на $V$, совместимых с симплектической формой $\omega$, и фоковское расслоение

$$
\mathscr{F}_{\mathscr{J}}:=\bigcup_{J \in \mathscr{J}(V)} F_{J} \rightarrow \mathscr{J}(V),
$$

мы не можем поднять естественное действие группы $\operatorname{Sp}(V)$ на $\mathscr{J}(V)$ до проективного действия этой группы на $\mathscr{F} \mathscr{J}$, накрывающего ее действие на базе $\mathscr{J}(V)$. Это запрещается теоремой Шейла-Березина. Поэтому приходится использовать другой подход к квантованию $\mathscr{T}$, основанный на соображениях из некоммутативной геометрии.

Напомним, что в изложенном выше дираковском подходе квантованию подвергались классические системы $(M, \mathscr{A})$, задаваемые фазовым пространством $M$ и алгеброй наблюдаемых $\mathscr{A}=\mathscr{A}_{\mathbb{C}}$, являющейся инволютивной алгеброй Ли, состоящей из гладких функций на $M$. Квантование такой системы задается неприводимым линейным представлением $r$ наблюдаемых из $\mathscr{A}$ замкнутыми линейными операторами, действующими в пространстве квантования $H$, переводящим скобку Пуассона $\{f, g\}$ наблюдаемых $f, g \in \mathscr{A}$ в коммутатор 
$[r(f), r(g)] / i$ отвечающих им операторов. В подходе Конна классическая система задается парой $(M, \mathfrak{A})$, где $M$ - снова фазовое пространство, а алгебра наблюдаемых $\mathfrak{A}$ есть ассоциативная инволютивная алгебра, состоящая из гладких функций на $M$. Квантованием такой системы по Конну называется неприводимое линейное представление $\pi$ наблюдаемых из $\mathscr{A}$ замкнутыми линейными операторами, действующими в пространстве квантования $H$, переводящее оператор внешнего дифференцирования $d$ в коммутатор с некоторым оператором симметрии $S$, где $S$ - самосопряженный оператор на $H$ с квадратом $S^{2}=I$. Иначе говоря,

$$
\pi: d f \mapsto[S, \pi(f)], \quad f \in \mathfrak{A} .
$$

Конновский подход можно сформулировать и на языке алгебр Ли. Для этого рассмотрим алгебру $\operatorname{Der}(\mathfrak{A})$ дифферениирований алгебры $\mathfrak{A}$, т. е. линейных отображений $\mathfrak{A} \rightarrow \mathfrak{A}$, удовлетворяющих правилу Лейбница. Алгебра $\operatorname{Der}(\mathfrak{A})$ является алгеброй Ли, поскольку коммутатор двух дифференцирований из этой алгебры снова является дифференцированием. В терминах алгебры $\operatorname{Der}(\mathfrak{A})$ квантование по Конну есть неприводимое представление алгебры Ли $\operatorname{Der}(\mathfrak{A})$ в алгебре Ли End $H$, наделенной коммутатором в качестве скобки Ли.

Если все наблюдаемые являются гладкими функциями на $M$ (как предполагалось выше), то между двумя подходами к квантованию нет большого различия. Действительно, дифференциал $d f$ наблюдаемой $f$ является симплектически двойственным к гамильтонову векторному полю $X_{f}$, что устанавливает связь между ассоциативной алгеброй наблюдаемых $\mathfrak{A} \ni f$ и алгеброй Ли гамильтоновых векторных полей $\mathscr{A} \ni X_{f}$ или двойственной к ней алгеброй Ли гамильтонианов $f$, порождающих векторные поля $X_{f}$. Оператор симметрии $S$ определяется в этом случае поляризащией

$$
H=H_{+} \oplus H_{-}
$$

пространства квантования $H$, т. е. разложением $H$ в прямую ортогональную сумму замкнутых бесконечномерных подпространств $H_{ \pm}$. Отвечающий поляризации оператор симметрии полагается равным $S= \pm I$ на $H_{ \pm}$. Этот оператор тесно связан с оператором комплексной структуры $J$ на $H$, задаваемым разложением (51), а именно, $S=i J$, так что $J= \pm i I$ на $H_{ \pm}$.

Однако в случае, если мы разрешим алгебре наблюдаемых $\mathscr{A}$ содержать негладкие функции, дираковское определение потеряет смысл. В конновском подходе дифференциал негладкой наблюдаемой $f \in \mathfrak{A}$ также не определен в классическом смысле, тем не менее его квантовый аналог

$$
d^{q} f:=[S, \pi(f)]
$$

может быть корректно определен.

Рассмотрим в качестве примера алгебру $\mathfrak{A}=L^{\infty}\left(S^{1}, \mathbb{C}\right)$ ограниченных функций на окружности $S^{1}$. Любая функция $f \in \mathfrak{A}$ определяет ограниченный опеpaтор умножения $M_{f}$ в гильбертовом пространстве $H=L^{2}\left(S^{1}\right)$, действующий по формуле

$$
M_{f}: h \in H \mapsto f h \in H .
$$

Оператор симметрии $S$ на $H$ задается преобразованием Гилъберта

$$
(S h)(\phi)=\frac{1}{2 \pi} \mathrm{P} . \mathrm{V} \cdot \int_{0}^{2 \pi} K(\phi, \psi) f(\psi) d \psi, \quad f \in H,
$$


где интеграл берется в смысле главного значения, т.е.

$$
\text { P.V. } \int_{0}^{2 \pi} K(\phi, \psi) f(\psi) d \psi:=\lim _{\epsilon \rightarrow 0}\left[\int_{0}^{\psi-\epsilon}+\int_{\psi+\epsilon}^{2 \pi}\right] K(\phi, \psi) f(\psi) d \psi .
$$

(Здесь и в дальнейшем мы отождествляем функции $f(z)$ на окружности $S^{1}$ с функциями $f(\phi):=f\left(e^{i \phi}\right)$ на отрезке $[0,2 \pi]$.) Ядро Гилъберта в формуле $(52)$ задается выражением

$$
K(\phi, \psi)=1+i \operatorname{ctg} \frac{\phi-\psi}{2} .
$$

Заметим, что при $\phi \rightarrow \psi$ оно ведет себя как $1+2 i /(\phi-\psi)$.

Дифференциал общей наблюдаемой $f \in \mathfrak{A}$ не определен в классическом смысле, но его квантовый аналог

$$
d^{q} f:=\left[S, M_{f}\right]
$$

корректно определен как оператор на $H$ (этот оператор корректно определен даже для функций из $\left.\mathrm{BMO}\left(S^{1}\right)\right)$. Для функций $f \in V^{\mathbb{C}}=H_{0}^{1 / 2}\left(S^{1}, \mathbb{C}\right)$ можно утверждать даже большее.

ПреДЛОЖЕНИЕ 11 [73]. Функиия $f$ принадлежит соболевскому пространству $V^{\mathbb{C}}$ тогда и только тогда, когда ее квантовый дифференииал $d^{q} f$ является оператором Гильберта-Шмидта на $H$ u, следовательно, на $V^{\mathbb{C}}$. Более того, норма Гильберта-Шмидта оператора $d^{q} f$ совпадает с соболевской нормой функции $f$.

Итак, квантовый дифференциал $d^{q} f:=\left[S, M_{f}\right]$ для функций $f \in V^{\mathbb{C}}$ является интегральным оператором на $V^{\mathbb{C}}$, задаваемым формулой

$$
\left(d^{q} f\right)(h)(\phi)=\frac{1}{2 \pi} \int_{0}^{2 \pi} k(\phi, \psi) h(\psi) d \psi, \quad h \in V^{\mathbb{C}},
$$

где

$$
k(\phi, \psi)=K(\phi, \psi)(f(\phi)-f(\psi)),
$$

a $K(\phi, \psi)$ - ядро Гильберта. При $\phi \rightarrow \psi$ ядро $k(\phi, \psi)$ ведет себя как

$$
\frac{f(\phi)-f(\psi)}{\phi-\psi} \text {. }
$$

Можно показать, что квазиклассический предел оператора (53), отвечающий взятию следа оператора (53) при $\phi=\psi$, совпадает с оператором умножения $h \mapsto f^{\prime} \cdot h$.

Тем самым, в рассмотренном примере квантование свелось, по существу, к замене производной ее конечно-разностным аналогом. Такое квантование, задаваемое соответствием

$$
\mathfrak{A} \ni f \mapsto d^{q} f: H \rightarrow H,
$$

Конн называет "квантовым исчислением" по аналогии с исчислением конечных разностей. 
Приведем без доказательства несколько конкретных примеров указанного соответствия между функциями $f \in \mathfrak{A}$ и операторами $d^{q} f$ на $H$ (подробнее об этом см. [89]):

1) дифференциал $d^{q} f$ является оператором конечного ранга тогда и только тогда, когда $f$ есть рациональная функция (теорема Кронекера);

2) дифференциал $d^{q} f$ является компактным оператором тогда и только тогда, когда функция $f$ принадлежит классу $\operatorname{VMO}\left(S^{1}\right)$;

3) дифференциал $d^{q} f$ является ограниченным оператором тогда и только тогда, когда функция $f$ принадлежит классу $\operatorname{BMO}\left(S^{1}\right)$.

Выше мы определили естественное действие группы $\mathrm{QS}\left(S^{1}\right)$ квазисимметричных гомеоморфизмов окружности на соболевском пространстве $V=H_{0}^{1 / 2}\left(S^{1}, \mathbb{R}\right)$. Это действие не является гладким и потому не допускает дифференцирования. Нам хотелось бы сопоставить пространству $\mathscr{T}$ классическую систему, в которой $\mathscr{T}$ играло бы роль фазового пространства, а в качестве алгебры наблюдаемых выступала бы алгебра Ли, ассоциированная с группой $\mathrm{QS}\left(S^{1}\right)$. Однако на классическом уровне такой алгебры Ли построить нельзя из-за негладкости действия $\mathrm{QS}\left(S^{1}\right)$. По этой причине мы не можем сопоставить $\mathscr{T}$ никакой естественной классической системы. Однако квантовую систему, ассоциированную с $\mathscr{T}$, мы построить можем.

Для этого определим сначала квантованное инфинитезимальное действие группы $\mathrm{QS}\left(S^{1}\right)$ на соболевском пространстве $V$, задавая его с помощью квантового дифференциала $d^{q}$, определяемого формулой (53):

$$
\mathrm{QS}\left(S^{1}\right) \ni f \mapsto d^{q} f: V \rightarrow V .
$$

Далее продолжим этот оператор на все фоковское пространство $F_{0}$, определяя его сначала на элементах базиса $P_{K}(z)$ из формулы (43) по правилу Лейбница, а затем продолжая по линейности на всю алгебру полиномов $\mathfrak{S}\left(W_{+}\right)$. Замыкание полученного оператора даст нам оператор $d^{q} f$ на фоковском пространстве $F_{0}=\widehat{\mathfrak{S ( W _ { + } )}}$. Искомая квантовая алгебра Ли наблюдаемых порождается построенными операторами $d^{q} f$ на $F_{0}$ с $f \in \operatorname{QS}\left(S^{1}\right)$. Мы обозначаем ее через $\operatorname{Der}^{q}(\mathrm{QS})$ и рассматриваем в качестве замены (не существующей) классической алгебры Ли группы $\mathrm{QS}\left(S^{1}\right)$.

Сравним изложенный способ построения квантовой системы, ассоциированной с $\mathscr{T}$, с дираковским квантованием системы $\left(\mathscr{J}_{\mathrm{HS}}(V), \operatorname{sp}_{\mathrm{HS}}(V)\right)$.

В случае системы $\left(\mathscr{J}_{\mathrm{HS}}(V), \operatorname{sp}_{\mathrm{HS}}(V)\right)$ :

1) мы стартуем с $\operatorname{Sp}_{\mathrm{HS}}(V)$-действия на $\mathscr{J}_{\mathrm{HS}}$;

2) затем, пользуясь теоремой Шейла-Березина, продолжаем это действие до проективного унитарного действия группы $\operatorname{Sp}_{\mathrm{HS}}(V)$ на фоковских пространствах $F(V, J)$;

3) инфинитезимальная версия этого действия дает проективное унитарное представление симплектической алгебры Гильберта-Шмидта $\operatorname{sp}_{\mathrm{HS}}(V)$ в фоковском пространстве $F_{0}$.

В случае универсального пространства Тейхмюллера $\mathscr{T}$ :

1) у нас имеется действие группы $\mathrm{QS}\left(S^{1}\right)$ на пространстве $V$; однако, в отличие от квантования $\mathscr{J}_{\mathrm{HS}}$, шаг $(2)$ в случае $\mathscr{T}$ невозможен, поскольку согласно теореме Шейла-Березина мы не можем продолжить действие $\mathrm{QS}\left(S^{1}\right)$ на фоковские пространства $F(V, J)$; 
2) вместо этого мы вводим квантованное инфинитезимальное действие группы $\mathrm{QS}\left(S^{1}\right)$ на $V$, задаваемое квантовыми дифференциалами $d^{q} f$;

3) продолжая операторы $d^{q} f$ на фоковское пространство $F_{0}$, мы получаем квантовую алгебру Ли $\operatorname{Der}^{q}(\mathrm{QS})$, порождаемую продолженными операторами $d^{q} f$.

Итак, конновское квантование универсального пространства Тейхмюллера $\mathscr{T}$ состоит из двух этапов.

I. Первичное квантование: построение квантованного инфинитезимального действия группы $\mathrm{QS}\left(S^{1}\right)$ на соболевском пространстве $V$, задаваемого посредством

$$
\operatorname{QS}\left(S^{1}\right) \ni f \mapsto d^{q} f=\left[S, M_{f}\right]: V \rightarrow V .
$$

II. Вторичное квантование: продолжение операторов $d^{q} f$ на фоковское пространство $F_{0}$ и построение квантовой алгебры наблюдаемых $\operatorname{Der}^{q}(\mathrm{QS})$, порождаемой операторами $d^{q} f \in \operatorname{End} F_{0}$ с $f \in \operatorname{QS}\left(S^{1}\right)$.

Принцип соответствия для построенного квантования пространства $\mathscr{T}$ состоит в том, что ограничение конновского квантования $\mathscr{T}$ на пространство нормализованных диффеоморфизмов $\mathscr{S} \subset \mathscr{T}$ дает нам дираковское квантование $\mathscr{S}$, построенное в п. 3.6 .

\section{Список литературы}

[1] W. Abikoff, "Augmented Teichmüller spaces", Bull. Amer. Math. Soc., 82:2 (1976), 333-334.

[2] W. Abikoff, "Degenerating families of Riemann surfaces", Ann. of Math. (2), 105:1 (1977), 29-44.

[3] У. Абикоф, Вещественно аналитическая теория пространства Тейхмюллера, Математика. Новое в зарубежной науке, 38, Мир, М., 1985, 119 с.; пер. с англ.: W. Abikoff, The real analytic theory of Teichmüller spaces, Lecture Notes in Math., 820, Springer, Berlin, 1980, vii+144 pp.

[4] W. Abikoff, "Oswald Teichmüller", Math. Intelligencer, 8:3 (1986), 8-16, 33.

[5] W. Abikoff, C. Corillon, J. Gilman, I. Kra, T. Weinstein, "Remembering Lipman Bers", Notices Amer. Math. Soc., 42:1 (1995), 8-25.

[6] L. Ahlfors, "Zur Theorie der Überlagerungsflächen", Acta Math., 65:1 (1935), 157-194.

[7] L. V. Ahlfors, "On quasiconformal mappings", J. Analyse Math., 3:1 (1953/1954), 1-58; рус. пер.: [11], 104-172.

[8] L. Ahlfors, "Curvature properties of Teichmüller's space", J. Anal. Math., 9:1 (1961/1962), 161-176.

[9] Л. Альфорс, Лекиии по квазиконформным отображениям, Мир, М., 1969, 133 с.; пер. с англ.: L. V. Ahlfors, Lectures on quasiconformal mappings, Van Nostrand Math. Stud., 10, D. Van Nostrand Co., Inc., Toronto-New York-London, 1966, v+146 pp.

[10] L. Ahlfors, Conformal invariants: topics in geometric function theory, McGraw-Hill Ser. in Higher Math., McGraw-Hill Book Co., New York-Düsseldorf-Johannesburg, 1973, ix+157 pp.

[11] Л. Альфорс, Л. Берс, Пространства римановых поверхностей и квазиконформные отображения, ИЛ, М., 1961, 177 с.

[12] L. V. Ahlfors, A. Beurling, "Invariants conformes et problèmes extrémaux", Comptes Rendus du Dixième Congrès des Mathematiciens Scandinaves 1946, Jul. Gjellerups Forlag, Copenhagen, 1947, 341-351. 
[13] П. П. Белинский, Общие свойства квазиконформных отображсений, Наука, Новосибирск, 1974, 98 с.

[14] Ф.А. Березин, Метод вторичного квантования, 2-е изд., Наука, М., 1986, 320 с.

[15] L. Bers, "Isolated singularities of minimal surfaces", Ann. of Math. (2), 53:2 (1951), 364-386.

[16] L. Bers, "Quasiconformal mappings and Teichmüller's theorem", Analytic functions, Princeton Univ. Press, Princeton, NJ, 1960, 89-119; рyc. пер.: [11], 9-50.

[17] L. Bers, "Universal Teichmüller space", Analytic methods in mathematical physics (Indiana Univ., Bloomington, IN, 1968), Gordon and Breach, New York, 1970, 65-83.

[18] L. Bers, "On boundaries of Teichmüller spaces and on Kleinian groups. I", Ann. of Math. (2), 91:3 (1970), 570-600.

[19] L. Bers, "An extremal problem for quasiconformal mappings and a theorem by Thurston", Acta Math., 141:1-2 (1978), 73-98.

[20] L. Bers, I. Kra (eds.), A crash course on Kleinian groups, Lectures given at a special session at the Annual Winter Meeting of the American Mathematical Society (San Francisco, CA, January 1974), Lecture Notes in Math., 400, Springer-Verlag, Berlin-New York, 1974, v+130 pp.

[21] R. Bowen, "Hausdorff dimension of quasi-circles", Inst. Hautes Études Sci. Publ. Math., 50:1 (1979), 11-25.

[22] M. J. Bowick, S. G. Rajeev, "The holomorphic geometry of closed bosonic string theory and Diff $S^{1} / S^{1} "$, Nuclear Phys. B, 293:2 (1987), 348-384.

[23] M. R. Chowdhury, "Landau and Teichmüller", Math. Intelligencer, 17:2 (1995), 12-14.

[24] A. Connes, Géométrie non commutative, InterEditions, Paris, 1990, 240 pp.

[25] P. Deligne, D. Mumford, "The irreducibility of the space of curves of given genus", Inst. Hautes Études Sci. Publ. Math., 36:1 (1969), 75-109.

[26] В.Н. Дубинин, "Симметризация в геометрической теории функций комплексного переменного", УМH, 49:1(295) (1994), 3-76; англ. пер.: V.N. Dubinin, "Symmetrization in the geometric theory of functions of a complex variable", Russian Math. Surveys, 49:1 (1994), 1-79.

[27] C. J. Earle, "On the Carathéodory metric in Teichmüller spaces", Discontinuous groups and Riemann surfaces (Univ. Maryland, College Park, MD, 1973), Ann. of Math. Studies, 79, Princeton Univ. Press, Princeton, NJ, 1974, 99-103.

[28] L. D. Faddeev, V. N. Popov, "Feynman diagrams for the Yang-Mills field", Phys. Lett. B, 25:1 (1967), 29-30.

[29] A. Fathi, F. Laudenbach, V. Poénaru (eds.), Travaux de Thurston sur les surfaces, Séminaire Orsay, Astérisque, 66-67, Soc. Math. France, Paris, 1979, 284 pp.

[30] A. Fletcher, V. Markovic, Quasiconformal maps and Teichmüller theory, Oxf. Grad. Texts Math., 11, Oxford Univ. Press, Oxford, 2007, viii+189 pp.

[31] В.В. Фок, Л. О. Чехов, "Квантовые пространства Тейхмюллера", ТМФ, 120:3 (1999), 511-528; англ. пер.: V.V. Fock, L. O. Chekhov, "A quantum Teichmüller space", Theoret. and Math. Phys., 120:3 (1999), 1245-1259.

[32] F.P. Gardiner, Teichmüller theory and quadratic differentials, Pure Appl. Math. (N. Y.), A Wiley-Interscience Publication. John Wiley \& Sons, Inc., New York, 1987, Xviii+236 pp.

[33] F. P. Gardiner, N. Lakic, Quasiconformal Teichmüller theory, Math. Surveys Monogr., 76, Amer. Math. Soc., Providence, RI, 2000, xx+372 pp.

[34] F.P. Gardiner, D.P. Sullivan, "Symmetric structures on a closed curve", Amer. J. Math., 114:4 (1992), 683-736.

[35] R. Goodman, N. R. Wallach, "Structure and unitary cocycle representations of loop groups and the group of diffeomorphisms of the circle", J. Reine Angew. Math., 347 (1984), 69-133. 
[36] T. Gotô, "Relativistic quantum mechanics of one-dimensional mechanical continuum and subsidiary condition of dual resonance model", Progr. Theoret. Phys., 46:5 (1971), 1560-1569.

[37] J. M. Gracia-Bondía, J. C. Várilly, H. Figueroa, Elements of noncommutative geometry, Birkhäuser Adv. Texts Basler Lehrbücher, Birkhäuser Boston, Inc., Boston, MA, 2001, xviii+685 pp.

[38] H. Grötzsch, "Über einige Extremalprobleme der konformen Abbildung. I, II", Berichte Leipzig, 80 (1928), 367-376, 497-502.

[39] R. Guo, "A survey of quantum Teichmüller space and Kashaev algebra", Handbook of Teichmüller space, v. IV, ed. A. Papadopoulos, Eur. Math. Soc., Zürich, 27 pp. (to appear); 2011, arXiv: 1107.5069.

[40] R. Guo, X. Liu, "Quantum Teichmüller space and Kashaev algebra", Algebr. Geom. Topol., 9:3 (2009), 1791-1824.

[41] R. A. Hidalgo, A. Vasil'ev, "Noded Teichmüller spaces", J. Anal. Math., 99:1 (2006), 89-107.

[42] E. D'Hoker, D. H. Phong, "Multiloop amplitudes for the bosonic Polyakov string", Nuclear Phys. B, 269:1 (1986), 205-234.

[43] J.H. Hubbard (ed.), Teichmüller theory and applications to geometry, topology, and dynamics, v. 1: Teichmüller theory, Matrix Editions, Ithaca, NY, 2006, xx+459 pp.

[44] A. Hurwitz, "Ueber Riemann'sche Flächen mit gegebenen Verzweigungspunkten", Math. Ann., 39 (1891), 1-61; Reprinted in: A. Hurwitz, Mathematische Werke. Bd. I: Funktionentheorie, Birkhäuser, Basel, 1932, 321-381.

[45] Y. Imayoshi, M. Taniguchi, An introduction to Teichmüller spaces, Springer-Verlag, Tokyo, 1992, xiv+279 pp.

[46] R. J. Milgram, R. C. Penner, "Riemann's moduli space and the symmetric groups", Mapping class groups and moduli spaces of Riemann surfaces (Göttingen, 1991/Seattle, WA, 1991), Contemp. Math., 150, Amer. Math. Soc., Providence, RI, 1993, $247-290$.

[47] J.A. Jenkins, "On the existence of certain general extremal metrics", Ann. of Math. (2), 66:3 (1957), 440-453.

[48] J.A. Jenkins, Univalent functions and conformal mapping, Ergeb. Math. Grenzgeb. N. F., 18, Springer-Verlag, Berlin-Göttingen-Heidelberg, 1958, vi+169 pp.

[49] J. Jost, Bosonic strings: a mathematical treatment, AMS/IP Stud. Adv. Math., 21, Amer. Math. Soc., Providence, RI; International Press, Somerville, MA, 2001, xii+95 pp.

[50] V.G. Kac, A.K. Raina, Bombay lectures on highest weight representations of infinite-dimensional Lie algebras, Adv. Ser. Math. Phys., 2, World Scientific Publishing Co., Inc., Teaneck, NJ, 1987, xii+145 pp.

[51] R. M. Kashaev, "Quantization of Teichmüller spaces and the quantum dilogarithm", Lett. Math. Phys., 43:2 (1998), 105-115.

[52] S.P. Kerckhoff, "The asymptotic geometry of Teichmüller space", Topology, 19:1 (1980), 23-41.

[53] S. P. Kerckhoff, W. P. Thurston, "Non-continuity of the action of the modular group at Bers' boundary of Teichmüller space", Invent. Math., 100:1 (1990), 25-47.

[54] А. А. Кириллов, Д. В. Юрьев, "Кэлерова геометрия бесконечномерного однородного многообразия $M=\operatorname{Diff}_{+}\left(S^{1}\right) / \operatorname{Rot}\left(S^{1}\right)$ ", Функи. анализ и его прил., 21:4 (1987), 35-46; англ. пер.: А. А. Kirillov, D. V. Yur'ev, "Kähler geometry of the infinite-dimensional homogeneous space $M=\operatorname{Diff}_{+}\left(S^{1}\right) / \operatorname{Rot}\left(S^{1}\right) "$, Funct. Anal. Appl., 21:4 (1987), 284-294.

[55] F. Klein, Ueber Riemann's Theorie der algebraischen Funktionen und ihrer Integrale, B. G. Teubner, Leipzig, 1882, 82 pp.; Reprinted in: F. Klein, Gesammelte mathematische Abhandlungen, v. 3, Springer, Berlin, 1923, 499-573. 
[56] I. Kra, "The Carathéodory metric on Abelian Teichmüller disks", J. Anal. Math., 40:1 (1981), 129-143.

[57] W. Kraus, "Über den Zusammenhang einiger Charakteristiken eines einfach zusammenhängenden Bereiches mit der Kreisabbildung", Mitt. Math. Semin. Univ. Giessen, 21 (1932), 1-28.

[58] С.Л. Крушкаль, Квазиконформные отображения и римановы поверхности, Наука, Новосибирск, 1975, 195 с.; англ. пер.: S.L. Krushkal, Quasiconformal mappings and Riemann surfaces, A Halsted Press Book. Scripta Series in Mathematics, V.H. Winston \& Sons, Washington, DC; John Wiley \& Sons, New York-Toronto-London, 1979, xii+319 pp.

[59] С. Л. Крушкаль, "Инвариантные метрики на пространствах Тейхмюллера", Сиб. матем. журн., 22:2 (1981), 209-212.

[60] S. L. Krushkal, "Invariant metrics on Teichmüller spaces and quasiconformal extendability of analytic functions", Ann. Acad. Sci. Fenn. Ser. A I Math., 10 (1985), 299-303.

[61] S. L. Krushkal, "Hyperbolic metrics on finite-dimensional Teichmüller spaces", Ann. Acad. Sci. Fenn. Ser. A I Math., 15:1 (1990), 125-132.

[62] С.Л. Крушкаль, Р. Кюнау, Квазиконформные отображения - новые методъ u приложения, Наука, Новосибирск, 1984, 162 с.; пер. с нем.: S. L. Krushkal, R. Kühnau, Quasikonforme Abbildungen - neue Methoden und Anwendungen, Teubner-Texte Math., 54, BSB B. G. Teubner, Leipzig, 1983, 169 pp.

[63] Г. В. Кузьмина, "Модули семейств кривых и квадратичные дифференциалы”, Тр. МИАН СССР, 139, 1980, 3-241; англ. пер.: G. V. Kuz'mina, "Moduli of families of curves and quadratic differentials", Proc. Steklov Inst. Math., 139 (1982), 1-231.

[64] M. A. Lavrentieff, "Sur une critère differentiel des transformations homeomorphes des domains à trois dimensions", Докл. АН СCCP, 20 (1938), 241-242.

[65] M. A. Lavrentieff, "Sur une classe de transformations quasi-conformes et sur les sillages gazeux", Докл. АН СССР, 20 (1938), 343-345.

[66] O. Lehto, Univalent functions and Teichmüller spaces, Grad. Texts in Math., 109, Springer-Verlag, New York, 1987, xii+257 pp.

[67] O. Lehto, K. I. Virtanen, Quasiconformal mappings in the plane, 2nd ed., Grundlehren Math. Wiss., 126, Springer-Verlag, New York-Heidelberg, 1973, viii+258 pp.

[68] Li-Xing Liu, "Invariant metrics in infinite-dimensional Teichmüller space", Complex Variables Theory Appl., 25:4 (1994), 337-349.

[69] H. Masur, "On a class of geodesics in Teichmüller space", Ann. of Math. (2), 102:2 (1975), 205-221.

[70] S. Nag, "Non-geodesic disks embedded in Teichmüller space", Amer. J. Math., 104:2 (1982), 399-408.

[71] S. Nag, The complex analytic theory of Teichmüller spaces, Canad. Math. Soc. Ser. Monogr. Adv. Texts, A Wiley-Interscience Publication. John Wiley \& Sons, Inc., New York, 1988, xiv+427 pp.

[72] S. Nag, "A period mapping in universal Teichmüller space", Bull. Amer. Math. Soc. (N.S.), 26:2 (1992), 280-287.

[73] S. Nag, D. Sullivan, "Teichmüller theory and the universal period mapping via quantum calculus and the $H^{1 / 2}$ space on the circle", Osaka J. Math., 32:1 (1995), 1-34.

[74] S. Nag, A. Verjovsky, "Diff $\left(S^{1}\right)$ and the Teichmüller spaces", Comm. Math. Phys., 130:1 (1990), 123-138.

[75] Y. Nambu, Duality and hydrodynamics, Lectures at the Copenhagen summer symposium, 1970.

[76] Z. Nehari, "Schwarzian derivatives and schlicht functions", Bull. Amer. Math. Soc., 55:6 (1949), 545-551. 
[77] C. Neumann, Vorlesungen über Riemann's Theorie der Abel'schen Integrale, B. G. Teubner, Leipzig, 1865; 2nd ed., 1884, 472 pp.

[78] R. Nevanlinna, Uniformisierung, Die Grundlehren der Mathematischen Wissenschaften in Einzeldarstellungen mit besonderer Berücksichtigung der Anwendungsgebiete, LXIV, Springer-Verlag, Berlin-Göttingen-Heidelberg, 1953, x+391 pp.

[79] J. Nielsen, "Untersuchungen zur Topologie der geschlossenen zweiseitigen Flächen", Acta Math., 50:1 (1927), 189-358.

[80] E. Noether, "Invariante Variationsprobleme", Gött. Nachr., 1918 (1918), 235-257.

[81] J.J. O'Connor, E.F. Robertson, Paul Julius Oswald Teichmüller, MacTutor History of Mathematics archive, Univ. of St. Andrews, 2009, http://www-history. mcs.st-andrews.ac.uk/Mathematicians/Teichmuller.html.

[82] J. J. O'Connor, E.F. Robertson, Lipman Bers, MacTutor History of Mathematics archive, Univ. of St. Andrews, 2002, http://www-history.mcs.st-andrews.ac.uk/ Mathematicians/Bers.html.

[83] M. Ohtsuka, Dirichlet problem, extremal length, and prime ends, Van Nostrand Reinhold Math. Ser., Van Nostrand Reinhold Co., New York, 1970, 326 pp.

[84] A. Papadopoulos (ed.), Handbook of Teichmüller theory, v. I, IRMA Lect. Math. Theor. Phys., 11, Eur. Math. Soc. (EMS), Zürich, 2007, viii+794 pp.; v. II, IRMA Lect. Math. Theor. Phys., 13, 2009, x+874 pp.; v. III, IRMA Lect. Math. Theor. Phys., 17, 2012, viii+366 pp.; v. IV (to appear).

[85] O. Pekonen, "Universal Teichmüller space in geometry and physics", J. Geom. Phys., 15:3 (1995), 227-251.

[86] H. Poincaré, "Sur les fonctions fuchsiennes", C. R. Acad. Sci. Paris, 92 (1881), 333-335; Reprinted in: Euvres, v. II, Gauthier-Villars, Paris, 1916, 1-4.

[87] Е. А. Полецкий, Б. В. Шабат, "Инвариантные метрики", Комплексный анализ многие переменные - 3, Итоги науки и техники. Соврем. проблемы матем. Фундам. напр., 9, ВИНИТИ, М., 1986, 73-125.

[88] A. M. Polyakov, "Quantum geometry of bosonic strings", Phys. Lett. B, 103:3 (1981), 207-210.

[89] S. Power, Hankel operators on Hilbert space, Res. Notes in Math., 64, Pitman (Advanced Publishing Program), Boston, MA-London, 1982, vii+87 pp.

[90] Э. Прессли, Г. Сигал, Группь петель, М., 1990, 456 с.; пер. с англ.: A. Pressley, G. Segal, Loop groups, 2nd rev. ed., Oxford Math. Monogr., Oxford Univ. Press, New York, 1988, viii+318 pp.

[91] T. Radó, "Über den Begriff der Riemannschen Fläche", Acta Litt. Sci. Szeged, 2 (1925), 101-121.

[92] B. Riemann, Grundlagen für eine allgemeine Theorie der Functionen einer veränderlichen complexen Größe, Inauguraldissertation, Göttingen, 1851; Reprinted in: Gesammelte mathematische Werke und wissenschaftlicher Nachlass, 2nd ed., B. G. Teubner, Leipzig, 1902, 3-48.

[93] B. Riemann, "Theorie der Abel'schen Functionen", J. Reine Angew. Math., 54 (1857), 101-155.

[94] H. L. Royden, "Automorphisms and isometries of Teichmüller space", Advances in the theory of Riemann surfaces (Stony Brook, NY, 1969), Ann. of Math. Stud., 66, Princeton Univ. Press, Princeton, NJ, 1971, 369-383.

[95] N. Schappacher, E. Scholz (eds.), "Oswald Teichmüller - Leben und Werk", Jahresber. Deutsch. Math.-Verein., 94:1 (1992), 1-39.

[96] G. Segal, "Unitary representations of some infinite dimensional groups", Comm. Math. Phys., 80:3 (1981), 301-342.

[97] А.Г. Сергеев, "Геометрическое квантование пространств петель", Совр. пробл. матем., 13, МИАН, М., 2009, 3-294; англ. изд.: А. Sergeev, Kähler geometry of loop spaces, MSJ Memoirs, 23, Math. Soc. Japan, Tokyo, 2010, xvi+212 pp. 
[98] A. Sergeev, "The group of quasisymmetric homeomorphisms of the circle and quantization of the universal Teichmüller space", SIGMA Symmetry Integrability Geom. Methods Appl., 5 (2009), paper 015, 20 pp., arXiv: 0902.1302.

[99] A. Sergeev, "Quantization of universal Teichmüller space", Geometry and quantization, Trav. Math., 19, Univ. Luxembourg, Luxembourg, 2011, 7-26.

[100] D. Shale, "Linear symmetries of free boson field", Trans. Amer. Math. Soc., 103:1 (1962), 149-167.

[101] H. Shiga, H. Tanigawa, "Grunsky's inequality and its applications to Teichmüller spaces", Kodai Math. J., 16:3 (1993), 361-378.

[102] А.Ю. Солынин, "Модули и экстремальные метрические задачи", Алгебра и анализ, 11:1 (1999), 3-86; англ. пер.: A. Yu. Solynin, "Moduli and extremal metric problems", St. Petersburg Math. J., 11:1 (2000), 1-65.

[103] K. Strebel, Quadratic differentials, Ergeb. Math. Grenzgeb. (3), 5, Springer-Verlag, Berlin, 1984, xii+184 pp.

[104] L. A. Takhtajan, Lee-Peng Teo, Weil-Petersson metric on the universal Teichmüller space, Mem. Amer. Math. Soc., 183, № 861, Amer. Math. Soc., Providence, RI, 2006, viii+119 pp.

[105] П. М. Тамразов, "Одна теорема об интегралах по кривым экстремальной длины”, Докл. АН Укр. ССР, 1 (1966), 51-54.

[106] O. Teichmüller, "Untersuchungen über konforme und quasikonforme Abbildung", Deutsche Math., 3 (1938), 621-678.

[107] O. Teichmüller, "Ungleichungen zwischen den Koeffizienten schlichter Funktionen", Sitzungsber. Preuß. Acad. Wiss. Phys.-Math. Kl., 1938, 363-375.

[108] O. Teichmüller, "Extremale quasikonforme Abbildungen und quadratische Differentiale", Abh. Preuß. Akad. Wiss. Math.-Nat. Kl., 22 (1939), 197 pp.

[109] O. Teichmüller, "Bestimmung der extremalen quasikonformen Abbildungen bei geschlossenen orientierten Riemannschen Flächen", Abh. Preuß. Akad. Wiss. Math.-Nat. Kl., 4 (1943), 42 pp.

[110] O. Teichmüller, "Veränderliche Riemannsche Flächen", Deutsche Math., 7 (1944), 344-359.

[111] O. Teichmüller, Gesammelte Abhandlungen, eds. L.V. Ahlfors, F.W. Gehring, Springer-Verlag, Berlin-New York, 1982, viii+751 pp.

[112] W.P. Thurston, "On the geometry and dynamics of diffeomorphisms of surfaces", Bull. Amer. Math. Soc., 19:2 (1988), 417-431.

[113] A. J. Tromba, Teichmüller theory in Riemannian geometry, Lectures Math. ETH Zurich, Birkhäuser Verlag, Basel, 1992, 220 pp.

[114] А.Ю. Васильев, "Гармонические свойства модуля семейства кривых и инвариантные метрики на пространстве Тейхмюллера”, Докл. РАН, 341:5 (1995), 583-584; англ. пер.: A. Yu. Vasil'ev, "Harmonic properties of the module of a family of curves and invariant metrics in Teichmüller space", Dokl. Math., 51:2 (1995), 237-238.

[115] А.Ю. Васильев, "Гомотопии кривых, отображений и метрика Тейхмюллера", Матем. заметки, 59:6 (1996), 923-926; англ. пер.: А. Yu. Vasil'ev, "Homotopy of curves and mappings and the Teichmüller metric", Math. Notes, 59:6 (1996), 668-671.

[116] А.Ю. Васильев, "Модули семейств кривых и инвариантные метрики на пространстве Тейхмюллера", Сиб. матем. журн., 37:5 (1996), 986-994; англ. пер.: A. Yu. Vasil'ev, "Moduli of families of curves and invariant metrics on Teichmüller space", Siberian Math. J., 37:5 (1996), 868-875.

[117] А.Ю. Васильев, "Инвариантные метрики и гармонические функционалы на пространстве Тейхмюллера", Алгебра и анализ, 9:1 (1997), 49-71; англ. пер.: A. Yu. Vasil'ev, "Invariant metrics and harmonic functionals on the Teichmüller space", St. Petersburg Math. J., 9:1 (1998), 33-48. 
[118] A. Vasiliev, Moduli of families of curves for conformal and quasiconformal mappings, Lecture Notes in Math., 1788, Springer-Verlag, Berlin, 2002, x+211 pp.

[119] K. Weierstrass, "Definition analytischer functionen einer Veränderlichen vermittelst algebraischer Differentialgleichungen", 1842, Mathematische Werke. I. Abhandlungen 1, Mayer \& Müller, Berlin, 1894, 75-84.

[120] A. Weil, "Modules des surfaces de Riemann", Séminaire Bourbaki, 10e année: 1957/1958, textes des conférences, exposés 152 à 168, Secrétariat mathématique, Paris, 1958, exp. 168, 7 pp.

[121] H. Weyl, Die Idee der Riemannschen Fläche, B. G. Teubner, Leipzig, 1913, x+169 pp.

[122] S. A. Wolpert, "The topology and geometry of the moduli space of Riemann surfaces", Workshop Bonn 1984, Lecture Notes in Math., 1111, Springer, Berlin, 1985, 431-451.

[123] S. Wolpert, "On the Weil-Petersson geometry of the moduli space of curves", Amer. J. Math., 107:4 (1985), 969-997.

[124] S. A. Wolpert, "Thurston's Riemannian metric for Teichmüller space", J. Differential Geom., 23:2 (1986), 143-174.

[125] S. Wolpert, "Chern forms and the Riemann tensor for the moduli space of curves", Invent. Math., 85:1 (1986), 119-145.

[126] N. M. J. Woodhouse, Geometric quantization, 2nd ed., Oxford Math. Monogr., Oxford Univ. Press, New York, 1992, xii+307 pp.

[127] П. Г. Зограф, Л.А. Тахтаджян, “Об уравнении Лиувилля, акцессорных параметрах и геометрии пространства Тейхмюллера для римановых поверхностей рода 0", Матем. сб., 132(174):2 (1987), 147-166; англ. пер.: P. G. Zograf, L.A. Takhtadzhyan, "On Liouville's equation, accessory parameters, and the geometry of Teichmüller space for Riemann surfaces of genus 0", Math. USSR-Sb., 60:1 (1988), 143-161.

[128] П. Г. Зограф, Л.А. Тахтаджян, “Об униформизации римановых поверхностей и метрике Вейля-Петерсона на пространствах Тейхмюллера и Шоттки", $M a$ тем. сб., 132(174):3 (1987), 304-321; англ. пер.: P. G. Zograf, L. A. Takhtadzhyan, "On uniformization of Riemann surfaces and the Weil-Petersson metric on Teichmüller and Schottky spaces", Math. USSR-Sb., 60:2 (1988), 297-313.

А. Ю. Васильев (А. Yu. Vasil'ev)

Поступила в редакцию

University of Bergen, Norway

27.01 .2013

E-mail: alexander.vasiliev@math.uib.no

А. Г. Сергеев (А. G. Sergeev)

Математический институт им. В. А. Стеклова РАН

E-mail: sergeev@mi.ras.ru 